\title{
Surgical versus non-surgical interventions for treating patellar dislocation (Review)
}

\author{
Smith TO, Donell S, Song F, Hing CB
}

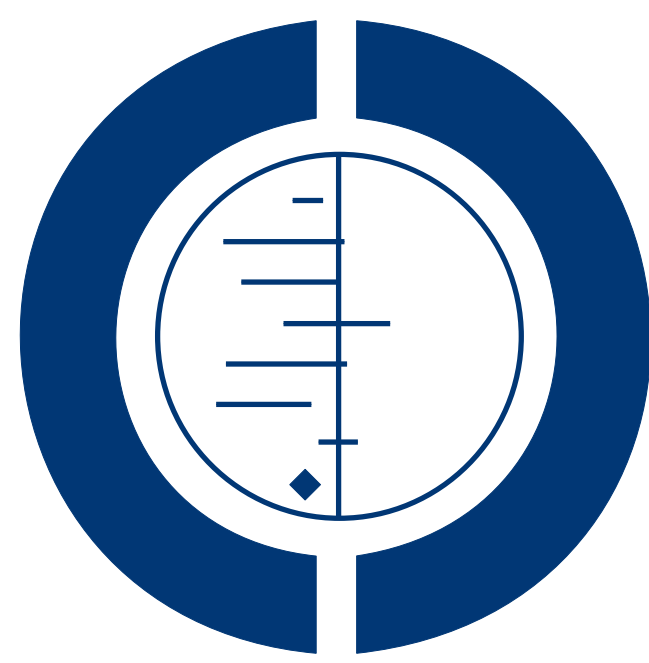

THE COCHRANE
COLLABORATION $^{\circledR}$

This is a reprint of a Cochrane review, prepared and maintained by The Cochrane Collaboration and published in The Cochrane Library 2015, Issue 2

http://www.thecochranelibrary.com

\section{WILEY}

Surgical versus non-surgical interventions for treating patellar dislocation (Review)

Copyright $\odot 2015$ The Cochrane Collaboration. Published by John Wiley \& Sons, Ltd. 
TABLE OF CONTENTS

HEADER . . . . . . . . . . . . . . . . . . . . . . . . . . . . . . . . . . . . . . . . . . . . .

ABSTRACT . . . . . . . . . . . . . . . . . . . . . . . . . . . . . . . . . . . . . . . . . . . . . .

PLAIN LANGUAGE SUMMARY . . . . . . . . . . . . . . . . . . . . . . . . . . . . . . . . . . . . . . . . . . . . .

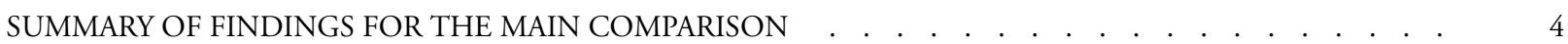

BACKGROUND . . . . . . . . . . . . . . . . . . . . . . . . . . . . . . . . . . . . . . . . . . . . . 9

OBJECTIVES . . . . . . . . . . . . . . . . . . . . . . . . . . . . . . . . . . . . . . . . . . . . . . . . .

METHODS . . . . . . . . . . . . . . . . . . . . . . . . . . . . . . . . . . . . . . . .

RESULTS . . . . . . . . . . . . . . . . . . . . . . . . . . . . . . . . . . . . . . . . . . . .

Figure 1. . . . . . . . . . . . . . . . . . . . . . . . . . . . . . . . . . . . . . . . 14

Figure 2. . . . . . . . . . . . . . . . . . . . . . . . . . . . . . . . . . . . . . .

Figure 3. . . . . . . . . . . . . . . . . . . . . . . . . . . . . . . . . . . . 18

Figure $4 . \quad$. . . . . . . . . . . . . . . . . . . . . . . . . . . . . . . . . . . . . . . .

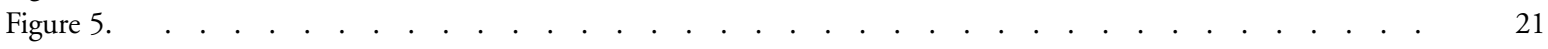

DISCUSSION . . . . . . . . . . . . . . . . . . . . . . . . . . . . . . . . . . . . . . . . . .

AUTHORS' CONCLUSIONS . . . . . . . . . . . . . . . . . . . . . . . . . . . . . . . . . . . . .

ACKNOWLEDGEMENTS . . . . . . . . . . . . . . . . . . . . . . . . . . . . . . . . . . . . . . .

REFERENCES . . . . . . . . . . . . . . . . . . . . . . . . . . . . . . . . . . . . . . 26

CHARACTERISTICS OF STUDIES . . . . . . . . . . . . . . . . . . . . . . . . . . . . . . . . . . . . . . . . .

DATA AND ANALYSES . . . . . . . . . . . . . . . . . . . . . . . . . . . . . . . . . . . . . . . . . . . . 44

Analysis 1.1. Comparison 1 Surgical versus non-surgical management, Outcome 1 Number of participants sustaining recurrent patellar dislocation. . . . . . . . . . . . . . . . . . . . . . . . . . . . . 46

Analysis 1.2. Comparison 1 Surgical versus non-surgical management, Outcome 2 Tegner activity score (0 to 10: best score).

Analysis 1.3. Comparison 1 Surgical versus non-surgical management, Outcome 3 KOOS (0 to 100: best outcome) at two years follow-up.

Analysis 1.4. Comparison 1 Surgical versus non-surgical management, Outcome 4 Lysholm score (0 to 100: best score) at two years (20 to 45 months) follow-up. . . . . . . . . . . . . . . . . . . . . . . . . . . . . . . . .

Analysis 1.5. Comparison 1 Surgical versus non-surgical management, Outcome 5 Hughston VAS patellofemoral score (28 to 100: best outcome).

Analysis 1.6. Comparison 1 Surgical versus non-surgical management, Outcome 6 Kujala patellofemoral disorders score (0 to 100 : best outcome).

Analysis 1.7. Comparison 1 Surgical versus non-surgical management, Outcome 7 Kujala patellofemoral disorders score $(0$ to 100: best outcome): subgroup analysis.

Analysis 1.8. Comparison 1 Surgical versus non-surgical management, Outcome 8 Return to former activities: work and sports.

Analysis 1.9. Comparison 1 Surgical versus non-surgical management, Outcome 9 Knee pain (VAS 0 to 10: worst outcome).

Analysis 1.10. Comparison 1 Surgical versus non-surgical management, Outcome 10 Patient satisfaction (reported good or excellent).

Analysis 1.11. Comparison 1 Surgical versus non-surgical management, Outcome 11 Number of participants sustaining recurrent patellar subluxation.

Analysis 1.12. Comparison 1 Surgical versus non-surgical management, Outcome 12 Number of participants sustaining any episode of instability.

Analysis 1.13. Comparison 1 Surgical versus non-surgical management, Outcome 13 Number of participants who underwent subsequent surgery. . . . . . . . . . . . . . . . . . . . . . . . . . . . . . . . . . . . . 56

APPENDICES

FEEDBACK

WHAT'S NEW

HISTORY

Surgical versus non-surgical interventions for treating patellar dislocation (Review)

Copyright $\odot 2015$ The Cochrane Collaboration. Published by John Wiley \& Sons, Ltd. 
SOURCES OF SUPPORT . . . . . . . . . . . . . . . . . . . . . . . . . . . . . . . . . 61

DIFFERENCES BETWEEN PROTOCOL AND REVIEW . . . . . . . . . . . . . . . . . . . . . . . . 62

INDEX TERMS $\quad . \quad$. . . . . . . . . . . . . . . . . . . . . . . . . . . . . . . . . . . . . . . . . . . . . . . . . . 62 


\title{
[Intervention Review] \\ Surgical versus non-surgical interventions for treating patellar dislocation
}

\author{
Toby O Smith ${ }^{1}$, Simon Donell ${ }^{1}$, Fujian Song ${ }^{1}$, Caroline B Hing ${ }^{2}$ \\ ${ }^{1}$ Faculty of Medicine and Health Sciences, University of East Anglia, Norwich, UK. ${ }^{2}$ Department of Trauma and Orthopaedic Surgery, \\ St George's Hospital, London, UK \\ Contact address: Toby O Smith, Faculty of Medicine and Health Sciences, University of East Anglia, Queen's Building, Norwich, \\ Norfolk, NR4 7TJ, UK. toby.smith@uea.ac.uk.
}

Editorial group: Cochrane Bone, Joint and Muscle Trauma Group.

Publication status and date: New search for studies and content updated (no change to conclusions), published in Issue 2, 2015.

Review content assessed as up-to-date: 13 October 2014.

Citation: Smith TO, Donell S, Song F, Hing CB. Surgical versus non-surgical interventions for treating patellar dislocation. Cochrane Database of Systematic Reviews 2015, Issue 2. Art. No.: CD008106. DOI: 10.1002/14651858.CD008106.pub3.

Copyright (C) 2015 The Cochrane Collaboration. Published by John Wiley \& Sons, Ltd.

\begin{abstract}
A B S T R A C T
Background

Patellar dislocation occurs when the patella disengages completely from the trochlear (femoral) groove. Following reduction of the dislocation, conservative (non-surgical) rehabilitation with physiotherapy may be used. Since recurrence of dislocation is common, some surgeons have advocated surgical intervention rather than non-surgical interventions. This is an update of a Cochrane review first published in 2011 .
\end{abstract}

Objectives

To assess the effects (benefits and harms) of surgical versus non-surgical interventions for treating people with primary or recurrent patellar dislocation.

\section{Search methods}

We searched the Cochrane Bone, Joint and Muscle Trauma Group's Specialised Register, the Cochrane Central Register of Controlled Trials (The Cochrane Library), MEDLINE, EMBASE, AMED, CINAHL, ZETOC, Physiotherapy Evidence Database (PEDro) and a variety of other literature databases and trial registries. Corresponding authors were contacted to identify additional studies. The last search was carried out in October 2014.

\section{Selection criteria}

We included randomised and quasi-randomised controlled clinical trials evaluating surgical versus non-surgical interventions for treating lateral patellar dislocation.

\section{Data collection and analysis}

Two review authors independently examined titles and abstracts of each identified study to assess study eligibility, extract data and assess risk of bias. The primary outcomes we assessed were the frequency of recurrent dislocation, and validated patient-rated knee or physical function scores. We calculated risk ratios (RR) for dichotomous outcomes and mean differences MD) for continuous outcomes. When appropriate, we pooled data.

Surgical versus non-surgical interventions for treating patellar dislocation (Review)

Copyright $\odot 2015$ The Cochrane Collaboration. Published by John Wiley \& Sons, Ltd. 


\section{Main results}

We included five randomised studies and one quasi-randomised study. These recruited a total of 344 people with primary (first-time) patellar dislocation. The mean ages in the individual studies ranged from 19.3 to 25.7 years, with four studies including children, mainly adolescents, as well as adults. Follow-up for the full study populations ranged from two to nine years across the six studies. The quality of the evidence is very low as assessed by GRADE (Grading of Recommendations Assessment, Development and Evaluation Working Group) criteria, with all studies being at high risk of performance and detection biases, relating to the lack of blinding.

There was very low quality but consistent evidence that participants managed surgically had a significantly lower risk of recurrent dislocation following primary patellar dislocation at two to five years follow-up (21/162 versus 32/136; RR 0.53 favouring surgery, 95\% confidence interval (CI) 0.33 to 0.87; five studies, 294 participants). Based on an illustrative risk of recurrent dislocation in 222 people per 1000 in the non-surgical group, these data equate to 104 fewer (95\% CI 149 fewer to 28 fewer) people per 1000 having recurrent dislocation after surgery. Similarly, there is evidence of a lower risk of recurrent dislocation after surgery at six to nine years (RR 0.67 favouring surgery, 95\% CI 0.42 to 1.08; two studies, 165 participants), but a small increase cannot be ruled out. Based on an illustrative risk of recurrent dislocation in 336 people per 1000 in the non-surgical group, these data equate to 110 fewer (95\% CI 195 fewer to 27 more) people per 1000 having recurrent dislocation after surgery.

The very low quality evidence available from single trials only for four validated patient-rated knee and physical function scores (the Tegner activity scale, KOOS, Lysholm and Hughston VAS (visual analogue scale) score) did not show significant differences between the two treatment groups.

The results for the Kujala patellofemoral disorders score (0 to 100: best outcome) differed in direction of effect at two to five years follow-up, which favoured the surgery group (MD 13.93 points higher, $95 \%$ CI 5.33 points higher to 22.53 points higher; four studies, 171 participants) and the six to nine years follow-up, which favoured the non-surgical treatment group (MD 3.25 points lower, $95 \%$ CI 10.61 points lower to 4.11 points higher; two studies, 167 participants). However, only the two to five years follow-up included the clear possibility of a clinically important effect (putative minimal clinically important difference for this outcome is 10 points).

Adverse effects of treatment were reported in one trial only; all four major complications were attributed to the surgical treatment group. Slightly more people in the surgery group had subsequent surgery six to nine years after their primary dislocation (20/87 versus 16/78; RR 1.06, 95\% CI 0.59 to 1.89, two studies, 165 participants). Based on an illustrative risk of subsequent surgery in 186 people per 1000 in the non-surgical group, these data equate to 11 more (95\% CI 76 fewer to 171 more) people per 1000 having subsequent surgery after primary surgery.

\section{Authors' conclusions}

Although there is some evidence to support surgical over non-surgical management of primary patellar dislocation in the short term, the quality of this evidence is very low because of the high risk of bias and the imprecision in the effect estimates. We are therefore very uncertain about the estimate of effect. No trials examined people with recurrent patellar dislocation. Adequately powered, multicentre, randomised controlled trials, conducted and reported to contemporary standards, are needed. To inform the design and conduct of these trials, expert consensus should be achieved on the minimal description of both surgical and non-surgical interventions, and the anatomical or pathological variations that may be relevant to both choice of these interventions and the natural history of patellar instability. Furthermore, well-designed studies recording adverse events and long-term outcomes are needed.

\section{PLAIN LANGUAGE SUMMARY}

\section{Surgical versus non-surgical treatment after kneecap dislocation}

\section{Background}

The patella or kneecap is a lens-shaped bone situated at the front of the knee. It is incorporated into the tendon of the quadriceps muscles of the thigh and moves within a groove at the lower end of the thigh bone (femur). Patellar dislocation occurs when the patella completely moves out of this groove. It typically occurs in young and physically active people with minimal trauma when they twist the bent knee with the foot fixed to the ground, for example, during sporting activities. The most common recurrent symptom reported by people is patella or knee cap instability. It may be associated with abnormal shape of the knee joint bones, weakness of the muscles around the hip or knees or tightness of soft tissues on the outside of the knee.

Surgical versus non-surgical interventions for treating patellar dislocation (Review)

Copyright $\odot 2015$ The Cochrane Collaboration. Published by John Wiley \& Sons, Ltd. 
When the patella dislocates, injury to the soft tissues of the knee joint occurs, which requires a period of rehabilitation. This may include treatments such as immobilisation and bracing (to limit knee movement), exercises, manual therapy, taping and electrotherapy modalities such as therapeutic ultrasound or electrical stimulation. However, some surgeons have suggested that people may have a better outcome if surgery is performed to repair or reconstruct the injured ligaments and muscles, re-shape the lower femur or change the position of where the patella attaches to the shinbone (tibia) to restrain the kneecap from dislocating again.

\section{Results of the search and description of studies}

This is an update of a previous Cochrane review. We searched the medical literature until October 2014 and we found six relevant studies (344 participants) that looked at the results of surgery compared with non-surgical treatment for people who had a kneecap dislocation. The studies allocated people to a surgical or non-surgical treatment group randomly. All study participants were being treated for a first-time dislocation. The mean ages in the individual studies ranged from 19 to 26 years, with four studies including children, mainly adolescents, as well as adults. Follow-up for study participants in the six included studies ranged from two to nine years.

\section{Key results}

The review found evidence of lower risk of repeated knee cap dislocation for those who underwent surgery compared with non-surgical intervention following first-time dislocation at two to five years follow-up. There was weaker evidence of a lower risk at six to nine years follow-up and an increased risk after surgery could not be ruled out. Very limited evidence for patient-rated knee and physical function outcome measures did not show a difference between the two groups. Although, evidence for an outcome measure that was specific to kneecap disorders was in favour of surgery at two to five years follow-up, the evidence at six to nine years follow-up did not show a benefit of surgery and tended to favour non-surgical treatment. One study only reported on adverse effects of treatment. This reported four major complications after surgery. Although slightly more people in the surgery group had subsequent surgery at six to nine years, the evidence for this outcome was inconclusive.

\section{Quality of the evidence}

These studies were small and had some weaknesses in their design and conduct. Overall, the quality of the evidence is very low and thus we were very uncertain about these findings.

\section{Conclusions}

Our review concludes that the evidence is not of sufficient quality to confirm a significant difference in outcome between surgical or non-surgical initial management of people who have dislocated their kneecap for the first time. There were no studies of people with recurrent patellar dislocation. Good quality research studies that are based on expert consensus about the condition and interventions and that involve a large number of people are required. 


\section{SUMMARY OF FINDINGSFOR THE MAINCOMPARISON [Explanation]}

\section{Surgical compared with non-surgical treatment for patellar dislocation}

Patient or population: people with first-time patellar dislocation

Settings: hospital (surgical) and/or hospital/rehabilitation centres (non-surgical)

Intervention: surgical procedures including medial patellofemoral ligament reconstruction and soft tissue repair to the patellofemoral joint

Comparison: non-surgical treatments including bracing/orthoses and exercise-based rehabilitation

\begin{tabular}{|c|c|c|c|c|c|c|}
\hline \multirow[t]{3}{*}{ Outcomes } & \multicolumn{2}{|c|}{ Illustrative comparative risks* $(95 \% \mathrm{CI})$} & \multirow{3}{*}{$\begin{array}{l}\text { Relative effect } \\
(95 \% \mathrm{CI})\end{array}$} & \multirow{3}{*}{$\begin{array}{l}\text { No of Participants } \\
\text { (studies) }\end{array}$} & \multirow{3}{*}{$\begin{array}{l}\text { Quality of the evidence } \\
\text { (GRADE) }\end{array}$} & \multirow[t]{3}{*}{ Comments } \\
\hline & Assumed risk & Corresponding risk & & & & \\
\hline & Non-surgical & Surgical & & & & \\
\hline $\begin{array}{l}\text { Number of partici- } \\
\text { pants sustaining recur- } \\
\text { rent patellar dislocation } \\
\text { Follow-up: } 2 \text { to } 5 \text { years }\end{array}$ & 222 per $1000^{2}$ & 118 per 1000 (73 to 194) & RR 0.53 (0.33 to 0.87 ) & $294(5)$ & $\begin{array}{l}\oplus \bigcirc \bigcirc \bigcirc^{3} \\
\text { very low }\end{array}$ & $\begin{array}{l}\text { Thus, based on an as- } \\
\text { sumed risk of } 222 \text { out } \\
\text { of } 1000 \text { people receiv- } \\
\text { ing non-surgical treat- } \\
\text { ment for primary patellar } \\
\text { dislocation having recur- } \\
\text { rent patellar dislocation } \\
\text { two to five years after their } \\
\text { dislocation, surgery re- } \\
\text { sulted in } 104 \text { fewer ( } 95 \% \\
\text { Cl } 149 \text { fewer to } 28 \text { fewer) } \\
\text { people per } 1000 \text { having a } \\
\text { recurrent dislocation dur- } \\
\text { ing this time }\end{array}$ \\
\hline
\end{tabular}

\section{Number of partici- 336 per $1000^{2}$}

pants sustaining recur-

rent patellar dislocation

Follow-up: 6 to 9 years
225 per 1000 (141 to RR 0.67 (0.42 to 1.08$) \quad 165(2)$

363) $\oplus \bigcirc \bigcirc \bigcirc^{3}$

very low
Thus, based on an assumed risk of 336 out of 1000 people who received non-surgical treatment for primary patellar dislocation having recur- 


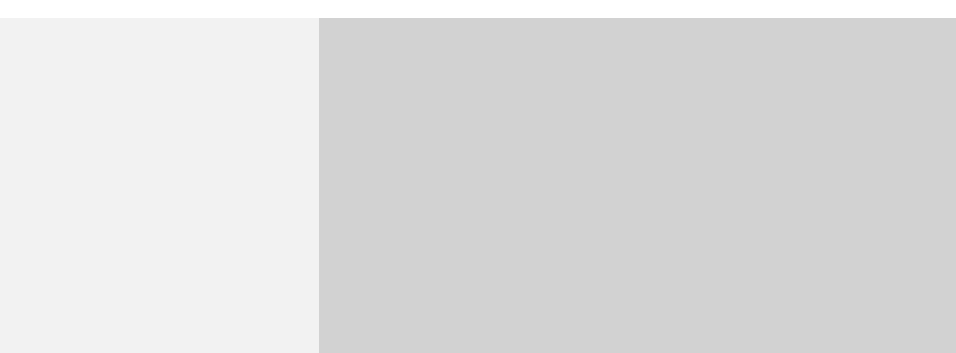

rent patellar dislocation six to nine years after their dislocation, surgery resulted in $\mathbf{1 1 1}$ fewer $(95 \%$ Cl 195 fewer to 27 more) people per 1000 having a recurrent dislocation during this time

Tegner activity score ${ }^{4} \quad$ The mean Tegner activity The mean Tegner activScale from 0 to 10 (higher score in the non-surgical ity score in the surgiscores $=$ better function) group was $\mathbf{5 . 0}$ points Follow-up: 6 to 9 years cal group was $\mathbf{0 . 0}$ points lower (1.15 point lower to 1.15 points higher)

$\oplus \bigcirc 00^{2}$ very low
The mean KOOS sub- The mean KOOS sub- Symptoms

$\mathrm{KOOS}^{4}$

Scale from: 0 to 100 (higher scores $=$ better function) Follow-up: 2 years

\begin{abstract}
80.2 to 92.3 points
\end{abstract}
6 points higher

\section{Sports and recreation} $3.60(-1.45 \text { to } 8.65)^{6}$

\section{7 (1)}

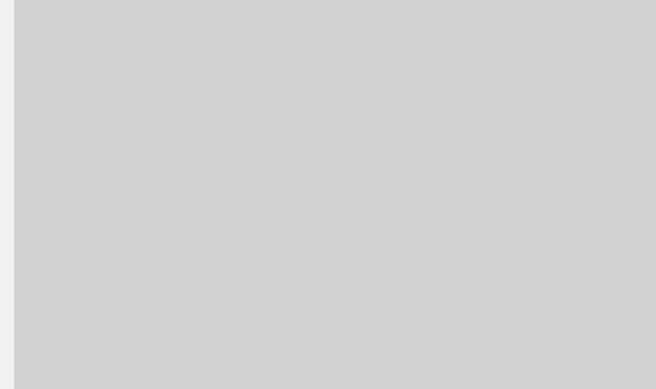

Higher scores indicate higher level of sporting/ activity participation

A second study reporting for six to nine years follow-up provided very low quality evidence ${ }^{3}$ of a 1 point difference in favour of non-surgical treatment (surgical versus non-surgical treatment: medians (interquartile range) 4 (3 to 5 ) versus 5 (4 to 6); reported $P$ value $=0.03$

Higher scores indicate better outcome for subsets: symptoms, pain activities of daily living, sports and recreation; and quality of life No subset scores were statistically significantly different between the treatments. The upper confidence intervals only very marginally overlap the proposed MCID for KOOS: 8 to $10^{7}$ 


\begin{tabular}{|c|c|c|}
\hline $\begin{array}{l}\text { Kujala patellofemoral } \\
\text { disorders score } \\
\text { Scale from: } 0 \text { to } 100 \\
\text { (higher scores = better } \\
\text { function) } \\
\text { Follow-up: } 2 \text { to } 5 \text { years }\end{array}$ & $\begin{array}{l}\text { The mean Ku- } \\
\text { jala patellofemoral disor- } \\
\text { ders score ranged across } \\
\text { non-surgical groups from } \\
\mathbf{6 9} \text { to } \mathbf{8 1} \text { points }\end{array}$ & $\begin{array}{l}\text { The mean } \\
\text { Kujala patellofemoral dis- } \\
\text { order score in the sur- } \\
\text { gical groups was } 13 \text {. } \\
\text { 93 points higher (5.33 } \\
\text { points higher to } 22.53 \\
\text { points higher) }\end{array}$ \\
\hline
\end{tabular}

Kujala patellofemoral The mean Ku- The mean Ku-

disorders score jala patellofemoral disor- jala patellofemoral disor-

Scale from: 0 to 100 ders score ranged across ders score in the surgical

(higher scores $=$ better non-surgical groups from groups was 3.25 points

function)

88 to 90 points

lower (10.61 points lower

Follow-up: 6 to 9 years

to 4.11 points higher)

$\oplus \bigcirc \bigcirc \bigcirc^{8}$

very low

$171(4)$

ity.

The confidence interval includes the putative MCID of 10 points 9 in favour of surgery. Thus this includes the possibility of a clinically important effect of surgery on outcome at 2 to 5 years assessed using this score

Lower scores indicate poorer functional capability.

The two trials contributing data here did not contribute data to the 2 to 5 years follow-up result The upper confidence interval only marginally includes the putative MCID of 10 points ${ }^{9}$ in favour of non-surgical treatment.

\begin{tabular}{|c|c|c|c|c|c|c|}
\hline $\begin{array}{l}\text { Adverse effects of treat- } \\
\text { ment } \\
\text { Incidence } \\
\text { Follow-up: } 2 \text { years ( } 20 \text { to } \\
45 \text { months) }\end{array}$ & $\begin{array}{l}\text { No post-randomisation } \\
\text { complications reported }\end{array}$ & $\begin{array}{l}\text { Four major complications } \\
\text { attributed to the surgical } \\
\text { group }\end{array}$ & & $125(1)$ & $\begin{array}{l}\oplus \bigcirc \bigcirc \bigcirc^{3} \\
\text { very low }\end{array}$ & $\begin{array}{l}\text { All four complications } \\
\text { were attributed to the sur- } \\
\text { gical management group. } \\
\text { The other five trials did not } \\
\text { record or report adverse } \\
\text { events }\end{array}$ \\
\hline $\begin{array}{l}\text { Subsequent requirement } \\
\text { for surgery (re-opera- } \\
\text { tions) for complications } \\
\text { Incidence }\end{array}$ & 186 per $1000^{2}$ & $\begin{array}{l}197 \text { per } 1000 \text { (110 to } \\
357)\end{array}$ & RR 1.06 (0.59 to 1.89) & $165(2)$ & $\begin{array}{l}\oplus \bigcirc \bigcirc \bigcirc^{3} \\
\text { very low }\end{array}$ & $\begin{array}{l}\text { There was no statistically } \\
\text { significant difference be- } \\
\text { tween groups for the } \\
\text { frequency of subsequent }\end{array}$ \\
\hline
\end{tabular}




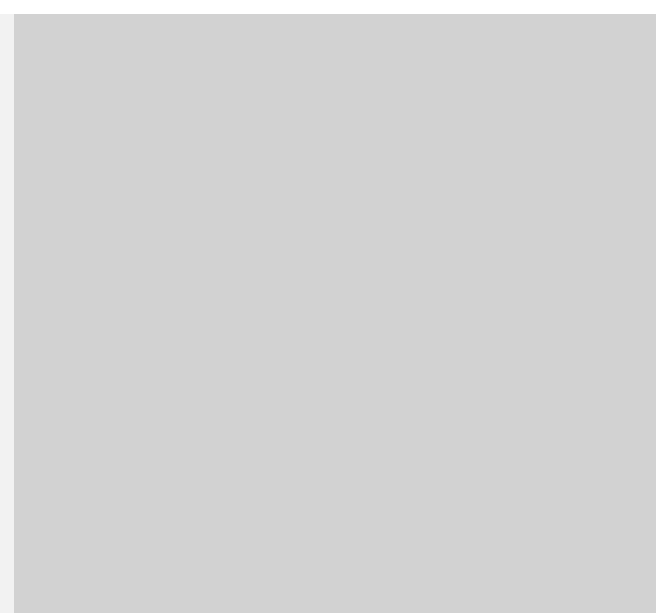

surgical interventions at 6 to 9 years

Based on an assumed risk of 186 out of 1000 people receiving non-surgical treatment for primary patellar dislocation having subsequent surgery six to nine years after their dislocation, surgery resulted in $\mathbf{1 1}$ more $(95 \% \mathrm{Cl} 76$ fewer to 171 more) people per 1000 having subsequent surgery during this time

*The basis for the assumed risk (e.g. the median control group risk across studies) is provided in footnotes. The corresponding risk (and its $95 \%$ confidence interval) is based on the assumed risk in the comparison group and the relative effect of the intervention (and its $95 \% \mathrm{Cl}$ ).

CI: Confidence interval;KOOS: Knee injury and Osteoarthritis Outcome Score; MCID: Minimal clinically important difference; RR: Risk Ratio; VAS: visual analogue scale

GRADE Working Group grades of evidence

High quality: Further research is very unlikely to change our confidence in the estimate of effect.

Moderate quality: Further research is likely to have an important impact on our confidence in the estimate of effect and may change the estimate.

Low quality: Further research is very likely to have an important impact on our confidence in the estimate of effect and is likely to change the estimate.

Very low quality: We are very uncertain about the estimate.

1. All six trials included in this review recruited only people with primary (first time) dislocation. The mean ages of participants in the individual trials ranged from 19.3 to 25.7 years. Four trials also recruited children, who were mainly adolescents

2. The basis for the assumed risk (e.g. the median control group risk across studies) in the footnotes

3. The evidence was downgraded two levels for serious study limitations and one level for imprecision

4. The Tegner activity scale was one of the four validated patient-rated knee and physical function scores for patellar dislocation outcomes for which data were reported by the included trials. The time period for Tegner was selected because data from two studies were available. The others were KOOS, Lysholm and Hughston VAS; each of which were reported by one trial only. Only the KOOS is presented because it was reported by a trial other than those presenting Tegner activity scale results. The very low quality evidence (125 participants, 1 trial) at two years (20 to 45 months) for both the Lysholm score (0 to 100: best outcome) and Hughston VAS score (28 to 100: best outcome) slightly favoured non-surgical treatment but the size of effect for both outcomes was probably not clinically relevant: MD -1.00, 95\% Cl -4.63 to 2.63 (Lysholm score); MD -2.80, 95\% Cl -6.70 to 1.10 (Hughston VAS score)

5. The evidence was downgraded one level for serious study limitations and two levels for serious imprecision 
The results of two of the five subsets provided here

7. " a MIC [Minimal clinically Important Change] of 8 to 10 is considered appropriate for the KO0S” http://www.koos.nu/koosfaq.html (accessed 17/01/2015)

. The evidence was downgraded two levels for serious study limitations and one level for inconsistency (statistically significant heterogeneity)

9. Whilst the MCID for the Kujala score has yet to be determined for the patellar dislocation population, a change exceeding 10 points is regarded as clinically meaningful for the anterior knee pain population (Bennell 2000; Crossley 2004) 


\section{B A C K G R O U N D}

\section{Description of the condition}

Patellar dislocation occurs when the patella disengages completely from the trochlear (femoral) groove, typically to the lateral side when the femur rotates internally on the tibia with the foot fixed on the ground. The patella may spontaneously slip back into its original position, or require manual reduction to push it back into place. The term "patellar instability" is used to include both patellar dislocation and subluxation (partial dislocation).

When the patella dislocates laterally, injury occurs to the soft tissues of the medial aspect of the knee joint, particularly to the medial patellofemoral ligament (Colvin 2008). This predisposes to subsequent episodes of patellar dislocation or subluxation, and eventually to degenerative change in the knee joint. As well as injury of the medial capsular structures, a range of anatomical factors may predispose to patellar instability; these include variations of limb alignment such as excessive valgus knee (Dath 2006; Smith 2011), or of architecture/geometry of the patella and lower femur, particularly of the trochlear groove such as trochlear dysplasia (Hing 2006), excessive lateral positioning of the attachment of the patellar tendon onto the shinbone (tibial tuberosity) or connective tissue laxity such as benign joint hypermobility syndrome (Beasley 2004).

The term 'primary patellar dislocation' refers to the first time a person experiences a patellar dislocation. Its incidence is highest in young and physically active people in the second and third decades of life (Buchner 2005; Kiviluoto 1986; Merchant 2007). The annual incidence of primary patellar dislocation has been estimated at 43 per 100,000 in children under 15 years (Nietosvaara 1994), with the incidence across all age groups much lower (estimated at 7 per 100,000 by Atkin 2000). Females are more likely to be affected than males (Fithian 2004). Women are more frequently more hypermobile than men (Scher 2010). Females also have a different muscle/body mass ratio (Strugnell 2014) meaning that they are more susceptible to injuries such as anterior cruciate ligament rupture and patellar dislocation (Hsiao 2010). Recurrent patellar dislocation can occur in $15 \%$ to $45 \%$ of primary dislocation cases (Cash 1988; Hawkins 1986; Woo 1998).

\section{Description of the intervention}

Following reduction of the patellar dislocation, people frequently undergo conservative (non-surgical) treatment consisting of physiotherapy and rehabilitation (Beasley 2004; Boden 1997; Woo 1998). This may include treatments such as immobilisation and bracing to limit knee movement, exercises, manual therapy, taping and electrotherapeutic modalities. Non-surgical management is frequently exercised-based, with the aim being to restore neuromusculoskeletal control of the patellofemoral joint at the hip, knee and foot or ankle through strengthening and muscle recruitment exercises and activities (Smith 2011). If muscles and softtissues are tight or restricted in length, most commonly the hamstrings, quadriceps, gastrocnemius or iliotibial band/tensor fascia lata, targeted stretching exercises are prescribed (Smith 2010; Smith 2011). Non-surgical management is most frequently delivered by a physiotherapist (Smith 2010; Smith 2011).

Some surgeons advocate surgical intervention for primary, or more frequently, recurrent dislocation (Donell 2006a; Fukushoma 2004). Such orthopaedic surgical interventions are of three main types:

1. Proximal patellar realignment soft tissue procedures, designed to repair or tighten the capsular soft tissues and tendinous soft tissues on the medial side of the knee (repair or medial plication) or reconstruct the ligamentous structures, particularly the medial patellofemoral ligament (MPFL) to resist lateral displacement of the patella (Conlan 1993; Hautamaa 1998). If the lateral capsular soft tissues appear too tight, they may be incised (lateral release).

2. Osseous (bony) procedures specifically for trochlear dysplasia (abnormal anatomy). This includes a trochleoplasty where the surgeon constructs a groove in the femur for the patella to move within (Dejour 1994; Donell 2006b). This may also include femoral or tibial osteotomy or abnormal or excessive rotation of the tibia or femur or tubercle transfer where, most commonly, the patella's attachment is medialised (moved more centrally) and distalised (moved downwards) to correct abnormal patellar tracking in the distal femur (Cosgarea 2002; Dath 2006; Dejour 1994).

3. Distal patellar realignment procedures. This can include a Roux-Goldthwaite procedure in which the surgeon alters where the patella attaches onto the tibia (Donell 2006a).

These interventions may be performed separately or in combination. The choice of surgical intervention will be influenced by the specific anatomical abnormalities predisposing the individual to their recurrent instability problem. Physiotherapy rehabilitation is most often commenced following any of the above surgical interventions to rehabilitate people post-operatively.

\section{How the intervention might work}

Non-surgical ('conservative') treatments including physiotherapy aim to restore knee range of motion and improve patellar stability with quadriceps strengthening exercises (Beasley 2004; Cosgarea 2002; Woo 1998). It has been suggested that one principal cause of recurrent patellar dislocation is weakness of the vastus medialis, one of the four muscles forming the quadriceps, (Dath 2006). By strengthening this muscle, it has been hypothesised that the patella will track more centrally in the trochlear groove, avoiding a more lateral position that may increase the likelihood of recurrent dislocation and instability symptoms (Donell 2006a). Similarly, strengthening muscle groups that control femoral internal 
rotation such as the glutei muscle complex, has been suggested to reduce lateral patellar tracking through maintenance of femoral neutrality during activity (Donell 2006a; Smith 2010; Woo 1998). Foot orthoses have also been recommended as a potential treatment adjunct, with the objective of controlling excessive tibial rotation, which may also influence patellar tracking through lateralisation of the patella's attachment on the tibia (Smith 2010). Finally, stretching shortened or tight soft tissues (such as of the hamstring, quadriceps, calf complexes) through exercise or manual technique including mobilisation or massage, in addition to the lateral retinaculum/iliotibial band/tensor fascia lata, has also been proposed to reduce lateralisation of the patella within the patellofemoral joint (Smith 2010).

Surgical interventions, as described above, offer repair or reconstruction of soft tissues, or procedures to deepen the trochlear groove or to realign the patellar tendon, to stabilise the patella in a more medial position. The hypothesis is that adding an appropriate surgical procedure in addition to their post-operative rehabilitation programme, these interventions will be more effective than conservative treatment alone in reducing the recurrent instability that may substantially limit functional capabilities and quality of life.

\section{Why it is important to do this review}

Some authors have suggested that surgical intervention should be considered rather than physiotherapy alone (Boden 1997; Guhan 2009). Others have written that surgical intervention may be no better in preventing recurrent dislocation and functional restoration than a conservative approach (Mears 2001; Nikku 1997a; Palmu 2008). Determining the optimal management approach for this population is important for a number of reasons. Firstly, there is a risk of cartilage lesions after repetitive subluxation and patellar dislocation. Repetitive injury of this nature can lead to early degenerative changes and osteoarthritis, resulting in longterm pain and disability (Donell 2006a). Secondly, patellar dislocation is more frequent in younger rather than older people (Buchner 2005; Kiviluoto 1986; Merchant 2007). Ascertaining the most appropriate management strategy for this population is important to minimise the impact of this condition on their lifestyles and subsequent activities.

The purpose of this systematic review is to inform clinical practice through the examination of the evidence from randomised trials comparing surgical to non-surgical treatment approaches following patellar dislocation.

\section{O B J E C T I VES}

To assess the effects (benefits and harms) of surgical versus nonsurgical interventions for treating people with primary or recurrent patellar dislocation.

\section{METHODS}

\section{Criteria for considering studies for this review}

\section{Types of studies}

Randomised and quasi-randomised (use of a method of allocating participants to a treatment that is not strictly random, e.g. by date of birth, hospital record number, alternation) controlled clinical trials (RCTs) evaluating surgical versus non-surgical interventions for treating patellar dislocation.

\section{Types of participants}

People of any age with a reported history of patellar dislocation, either primary or recurrent, recorded either as a historical account from the participants, or observed by a healthcare professional. We excluded trials that recruited participants who presented with anterior knee pain or patellar subluxation rather than a clear, convincing history or evidence of a patellar dislocation.

\section{Types of interventions}

Non-surgical intervention, or conservative management, is the control intervention in this review. Non-surgical treatment strategies following patellar dislocation include: a period of immobilisation, bracing or splinting, manual therapy, exercise-based treatments, education and advice, electrotherapeutic modalities and taping techniques.

Surgical treatment strategies include: medial reefing, quadricepsplasty, lateral release, tibial tubercle transfer, Roux-Goldthwaite procedures, trochleoplasty, medial patellofemoral ligament repair or reconstruction.

\section{Types of outcome measures}

The clinical and radiological outcome measures described below were assessed.

\section{Primary outcomes}

- Recurrent dislocation

- Validated patient-rated knee and physical function scores for patellar dislocation outcomes (Paxton 2003), e.g. the Lysholm score (Lysholm 1982), the Tegner activity score (Tegner 1985), the Hughston visual analogue score (VAS) (Flandry 1991) and the Short Form-12 (Ware 1996)

- Specific tool for appraising patella disorders: the Kujala score (Kujala 1993)

These outcomes were assessed at a minimum of one year after treatment, and analysed for each study time point reported in the respective papers. 


\section{Secondary outcomes}

- Other knee function and activity scores

- Return to former activities: work and sports

- Knee pain during activity or at rest, as measured using a VAS or similar

- Adverse events (complications), e.g. deep or superificial infection, nerve palsy, allergies, rash or abrasion from taping or orthoses

- Range of motion

- Patient-reported satisfaction such as measured with Likert scale, VAS or any other validated score

- Patient-reported instability symptoms

- Subsequent requirement for knee surgery (re-operations) for complications such as infection, or mechanical instability

These outcomes were assessed at each follow-up time point presented within the included studies, at a minimum of one year after treatment.

\section{Search methods for identification of studies}

\section{Electronic searches}

We searched the following databases.

- Cochrane Bone, Joint and Muscle Trauma Group's

Specialised Register (13 October 2014)

- Cochrane Central Register of Controlled Trials

(CENTRAL) (in The Cochrane Library 2014, Issue 9)

- MEDLINE (1950 to October Week 12014 )

- MEDLINE In-Process \& Other Non-Indexed Citations

(10 October 2014)

- EMBASE (1980 to 2013 Week 39)

- Allied and Complementary Medicine (AMED) (1985 to

October Week 3 2014)

- Cumulative Index to Nursing and Allied Health Literature (CINAHL) (1981 to October Week 3 2014)

- Health Management Information Consortium (to October Week 3 2014)

- Zetoc (to October Week 3 2014)

- Physiotherapy Evidence Database (PEDro) (October Week $32014)$

- Open Grey (System for Information on Grey Literature in Europe) (October 2014)

- WHO International Clinical Trials Registry Platform, Current Controlled Trials, UKCRN Portfolio Database, National Technical Information Service and the UK National Research Register Archive (October Week 32014 )

There were no constraints based on language or publication status. In MEDLINE we combined a subject-specific search with the Cochrane Highly Sensitive Search Strategy for identifying randomised trials in MEDLINE (sensitivity-maximising version)
(Lefebvre 2011). The EMBASE subject-specific search was combined with the Scottish Intercollegiate Guidelines Network ( SIGN) RCT filter. Details of search strategies for all databases are shown in Appendix 1,

\section{Searching other resources}

We searched conference proceedings from the British Orthopaedic Association Annual Congress, the British Trauma Society meetings, the European Federation of National Associations of Orthopaedics and Traumatology (EFORT) and the British Association for Surgery of the Knee via the supplements of the Bone and Joint Journal (October Week 3 2014). We also searched bibliographies of relevant articles and contacted trial investigators in this area.

\section{Data collection and analysis}

\section{Selection of studies}

Two review authors (TS and $\mathrm{CH}$ ) independently selected the potentially eligible articles from citation titles and, if available, abstracts. Upon obtaining full articles, the same two authors independently performed the study selection. In cases of disagreement of paper inclusion/exclusion, a consensus was reached through discussion. Had that not been possible, we would have sought arbitration from a third author (SD).

\section{Data extraction and management}

Two review authors (TS and $\mathrm{CH}$ ) independently extracted data from trial reports. We contacted corresponding authors when key information was missing. In cases of disagreement, we sought consensus through discussion or adjudication by a third author (SD). After the individual review authors had extracted the relevant data, these were collated to form a single, agreed and completed data extraction form with all the included study's characteristics and results. This presented all key trial data and participant information from the included articles.

\section{Assessment of risk of bias in included studies}

Two review authors (TS and $\mathrm{CH}$ ) independently assessed the risk of bias of the included studies using The Cochrane Collaboration's 'Risk of bias' tool (Higgins 2011). This consists of five domains: selection bias; performance bias; attrition bias; detection bias; and reporting bias. Risk of bias was categorised as low, unclear or high for each of the included studies. When no information was given by an included study, the review authors assumed that the study was unlikely to satisfy the criteria and therefore was given a rating of "high" risk of bias. When differences between the ratings of the 
two assessors could not be resolved through discussion, we asked a third author (SD) to adjudicate.

\section{Measures of treatment effect}

Treatment effects were measured using risk ratios (RR) for binary data and mean differences (MD) for continuous data. Should different scales or tools have been used to measure the same continuous outcome, we would have calculated standardised mean differences (SMDs). Ninety-five per cent confidence intervals were used throughout.

Measurement of treatment effect time points were categorised as: short term (less than or equal to two years post randomisation); medium term (two to nine years post randomisation); and long term (10 years or more post randomisation). Where studies presented several follow-up periods, we extracted and analysed data to inform short-, medium- and long-term results.

\section{Unit of analysis issues}

The unit of randomisation in the majority of trials included in this review was the individual participant. Exceptionally, as in the case of trials including people with bilateral patellar dislocations, data for trials may be presented for dislocations or knees rather than an individual person. Where such unit of analysis issues arose and appropriate corrections were not made, we presented the data for such trials only when the disparity between the units of analysis and randomisation was small.

\section{Dealing with missing data}

Corresponding authors were contacted in respect of any missing key information from their publications. Where appropriate, we performed intention-to-treat analyses to include all people randomised to the intervention groups. We investigated the effect of drop-outs and exclusions by conducting worst and best scenario analyses. We were alert to the potential mislabelling or misidentification of standard errors and standard deviations. Unless missing standard deviations could be derived from confidence interval data, we did not impute assumed values.

\section{Assessment of heterogeneity}

We appraised the clinical diversity in terms of participants, interventions and outcomes for the included studies. We assessed statistical heterogeneity by visual inspection of the forest plot and by using the $\mathrm{I}^{2}$ and $\mathrm{Chi}^{2}$ statistical tests.

\section{Assessment of reporting biases}

We assessed outcome reporting bias by considering the effects of missing data on measured outcomes. Had sufficient data been available (from at least 10 trials), we would have assessed publication bias using funnel plots.

\section{Data synthesis}

When judged appropriate, we pooled results from individual studies in meta-analyses using fixed- or random-effects models (depending on the results of heterogeneity tests) with $95 \%$ confidence intervals (CI). We adopted a fixed-effect model when there was no evidence of statistical heterogeneity ( $\mathrm{I}^{2}$ less than or equal to $30 \%$ and $\mathrm{Chi}^{2} \mathrm{P}>0.01$ ). We adopted a random-effects model where there was no evidence of methodological diversity such as cohort, intervention or trial procedure, but statistical heterogeneity was evident that could not be readily explained (as denoted with a $\mathrm{I}^{2}>$ $30 \%$ and $\mathrm{Chi}^{2} \mathrm{P}$ value equal to or less than 0.01 ). We were able to pool data in this review to determine short-, medium- and longterm outcomes.

\section{Subgroup analysis and investigation of heterogeneity}

Where appropriate, short-term data and medium-term follow-up data were presented under each comparison and pooled as separate subgroups allowing tests for subgroup differences. We also undertook a limited subgroup analysis comparing results of males versus females following surgical and non-surgical management. To test whether the subgroups were statistically significantly different from one another, we inspected the overlap of confidence intervals and performed the test for subgroup differences available in RevMan.

Should data become available in a future update, we plan to carry out subgroup analyses to assess the difference in outcome between participants over the age of 16 years (adults) and those younger than 16 years (children); those who are hypermobile versus nonhypermobile, in order to investigate whether these are important prognostic variables in this patient group. We will also assess the outcomes of patients who received treatment following primary dislocation compared with patients who were managed after recurrent patellar dislocation. We will also assess for a difference in outcome between different surgical treatments e.g. whether there is a difference in outcomes between repair versus reconstruction of MPFL. We do not intend to analyse the effect of timing of surgery or conservative intervention in relation to the time since the patient's primary patellar dislocation.

\section{Sensitivity analysis}

We undertook sensitivity analyses to examine the impact of including trials at high risk of bias due to lack of allocation concealment. We planned to undertake a sensitivity analysis of trials where the population was poorly defined; however, this was not a limitation within the included trials and therefore was not undertaken.

\section{Summary of findings}

We summarised the evidence available for the three primary outcomes listed in Types of outcome measures and incidence of complications (adverse effects of treatment) and subsequent requirement for surgery in a 'Summary of findings' table. We used the 
GRADE approach to determine the quality of evidence for each outcome (very low, low, moderate or high), as recommended by The Cochrane Collaboration (Higgins 2011).

\section{RES U L T S}

\section{Description of studies}

\section{Results of the search}

For this update we screened a total of 714 records from the following databases: Cochrane Bone, Joint and Muscle Trauma Group Specialised Register (3), CENTRAL (67), MEDLINE (66), EMBASE (61), AMED (96), CINAHL (73), Health Management Information Consortium (0), Zetoc (344), PEDro (4) and Open Grey (0). A further 181 trials were identified from trial registers. We also found six potentially eligible studies from reviewing the reference lists of potentially eligible papers.
The search update resulted in the identification of four new studies. Of these, two trials were selected for inclusion (Bitar 2012; Petri 2013), one study was excluded (Apostolovic 2011) and one is an ongoing trial (ISRCTN39959729). No studies are awaiting assessment.

Subsequent to the publication of the previous review (Hing 2011), one paper originally considered to be reporting a separate trial (Palmu 2008) was confirmed (Donell 2014) to be report of a children-only subgroup analysis of Nikku 1997. Therefore Palmu 2008 now appears under Nikku 1997.

Overall, there are now six included studies (Bitar 2012; Camanho 2009; Christiansen 2008; Nikku 1997; Petri 2013; Sillanpaa 2009), eight excluded studies (Apostolovic 2011; Arnbjörnsson 1992; Buchner 2005; Cash 1988; Marcacci 1995; Savarese 1990; Sillanpää 2008a; Sillanpää 2008b), one ongoing trial (ISRCTN39959729) and no studies awaiting assessment.

Further details of the process of screening and selecting studies for inclusion in the review are illustrated in Figure 1. The results from the previous searches (up to 2010) are shown in Appendix 2. 
Figure I. Study flow diagram
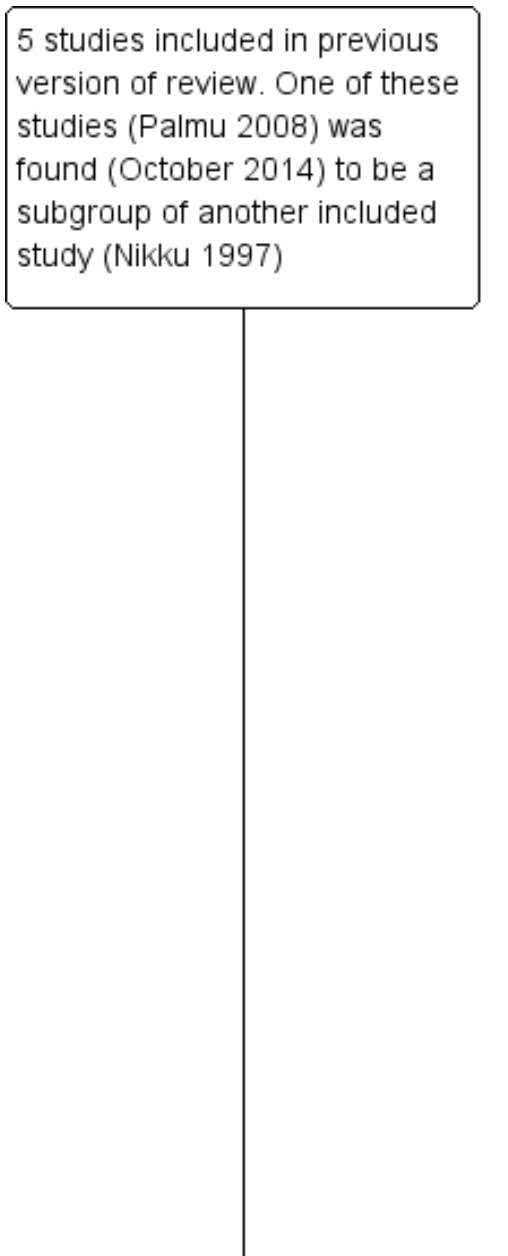
subgroup of another included

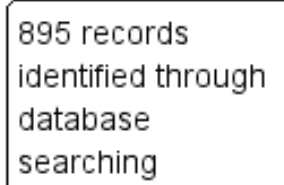

6 additional records identified through other sources

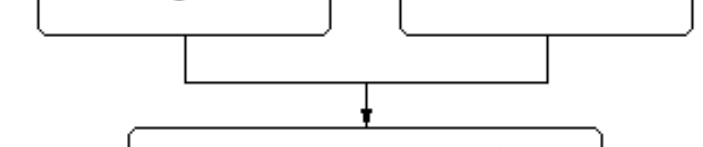

806 records after duplicates removed

802 records

excluded screened
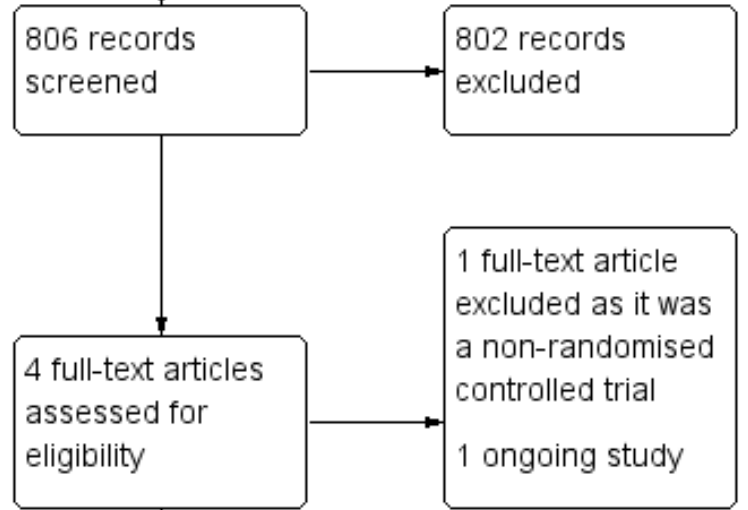

TOTAL of 6 studies included in qualitative synthesis

2 NEW studies

included 


\section{Included studies}

We included six trials published between 1997 and 2013. They were all written in English. Two studies were conducted in Finland (Nikku 1997; Sillanpaa 2009), two in Brazil (Camanho 2009; Bitar 2012), one in Germany (Petri 2013) and one in Denmark (Christiansen 2008).

\section{Randomisation procedure}

Five studies reported that they were randomised trials (Camanho 2009; Christiansen 2008; Sillanpaa 2009; Bitar 2012; Petri 2013) and one (Nikku 1997) was quasi-randomised by odd or even birth year.

\section{Participant demographic characteristics}

In total, 344 participants were recruited. Of the 334 participants for whom demographic data are available, 180 people (98 females and 82 males) were allocated surgery and 154 people (70 females and 84 males) were allocated non-surgical intervention. The mean ages in the surgery groups ranged from 19.5 years (Nikku 1997) to 27.2 years (Petri 2013). The mean age in the non-surgical groups ranged from 19.1 years (Nikku 1997) to 24.6 years (Camanho 2009). In the individual trials, the mean age ranged from 19.3 years in Nikku 1997 to 25.7 years in Camanho 2009; and the percentage of females from $7.5 \%$ in Sillanpaa 2009, which included military recruits, to $65.6 \%$ in Nikku 1997 . Four trials included children, who were mainly adolescents, as well as adults (Bitar 2012; Camanho 2009; Christiansen 2008; Nikku 1997). The youngest participants were nine years old (Nikku 1997) and the oldest, who was an outlier, was 74 years (Camanho 2009).

Nikku 1997 reported the outcomes of 127 knees in 125 participants, whilst Bitar 2012 reported the outcomes of 41 knees in 39 participants. In Bitar 2012, presenting trial data by patellar dislocation was unavoidable with the exception of knee-specific outcomes such as the incidence of recurrent instability/dislocation. Only Bitar 2012 and Nikku 1997 made reference to whether their participants presented with joint hypermobility. Bitar 2012 reported that no patellar hypermobility was detected and Nikku 1997 stated that one participant in each group presented with ligament laxity as assessed using the Beighton score (Carter 1964).

\section{Patellar dislocation and eligibility criteria characteristics}

All six studies recruited participants who had sustained a primary patellar dislocation; thus none of the studies recruited people who had experienced recurrent or previous patellar dislocations. The diagnosis of patellar dislocation was made during initial clinical examination within each of the RCTs, on the basis of a variety of different combinations of signs and symptoms. These inclusion criteria included: patellar dislocation requiring reduction in two studies (Christiansen 2008; Camanho 2009), a history of acute knee trauma in five studies (Bitar 2012; Camanho 2009; Nikku 1997; Petri 2013; Sillanpaa 2009), and intra-articular haematoma, tenderness on the medial epicondyle and positive lateral patellar apprehension test results in Christiansen 2008. Magnetic resonance imaging was used as part of the eligibility screening of potential participants in one study (Sillanpaa 2009). All participants in Christiansen 2008 and Petri 2013 underwent arthroscopy to aid diagnosis.

The main exclusion criteria were the presence of a large osteochondral fracture in five studies (Bitar 2012; Camanho 2009; Nikku 1997; Petri 2013; Sillanpaa 2009), an inability to followup the planned treatment regimens in two studies (Bitar 2012; Christiansen 2008), prior knee surgery in three studies (Bitar 2012; Christiansen 2008; Nikku 1997) and a previously reported patellar dislocation or instability in all six studies (Bitar 2012; Camanho 2009; Christiansen 2008; Nikku 1997; Petri 2013; Sillanpaa 2009). Other exclusion criteria were the co-existence of a significant tibiofemoral ligament injury requiring surgical fixation (Bitar 2012), people with conditions associated with serious neuromuscular or congenital diseases (Bitar 2012), a history of a non-traumatic event such as walking or squatting with 'moderate' stress on the knee and in the absence of acute pain in the knee (Bitar 2012), open injury (Petri 2013) or women who were pregnant or lactating (Petri 2013).

\section{Non-surgical management}

Non-surgical management in all studies consisted of initial immobilisation in a cast, splint or locked orthosis, followed by active mobilisation with physiotherapy. There was variation in the duration of immobilisation and in components of the physiotherapy programmes (see Characteristics of included studies). Whilst all participants in Christiansen 2008 and Petri 2013 underwent arthroscopy prior to randomisation, this was a diagnostic arthroscopic procedure and not a therapeutic arthroscopy. Of note in Sillanpaa 2009, all participants in the non-operative group received knee aspiration to relieve pain and four underwent arthroscopic removal of an osteochrondral fragment. All these studies were included given the non-corrective nature of these procedures.

\section{Surgical management}

The predominant operative intervention was repair or reconstruction of the soft tissues of the medial aspect of the knee joint. Both Camanho 2009 and Christiansen 2008 reported that all participants solely received a MPFL suture repair. Nikku 1997 reported 
that all participants allocated to surgery in their trial received either a medial reefing with an MPFL augmentation using adductor magnus (six participants) or medial reefing with a lateral release (54 participants). Petri 2013 reported that their surgical intervention was repair of the medial soft tissues and a "MPFL-plastic" procedure was not undertaken. Whilst they acknowledged that a lateral release was optional, they did not stipulate the frequency with which this procedure was undertaken. Sillanpaa 2009 allocated 14 participants in the surgical group to receive a combined medial reefing procedure and MPFL suture repair; a Roux-Goldthwaite procedure for four participants, and an arthroscopic repair was also required for an osteochondral fracture in six people. In Bitar 2012, the surgical procedure was an MPFL reconstruction using a medial slip of the patellar ligament, which was then sutured to the distal aspect of the vastus medialis muscle.

All participants allocated to the surgical management strategies received a period of post-operative rehabilitation. The post-operative rehabilitation programme used in each study was identical to that used in the non-operative group, with the exception of Camanho 2009 who, rather than immobilising the participants in an inguinal-malleolar splint for three weeks, permitted their surgical patients to wear a removable immobiliser for three weeks and to commence passive knee range of motion exercises during this early post-operative period.

\section{Follow-up time points and outcome measures}

The maximum follow-up was two years in two studies ( Christiansen 2008; Petri 2013). The mean follow-up was 44 months, range 24 to 61 months, in Bitar 2012. Follow-up in Camanho 2009 was after two years and before five years, the mean follow-ups in the surgical and non-surgical groups being 40.4 and 36.3 months respectively. Nikku 1997 presented data at mean follow-up periods of 25 months (range 20 to 45 months), seven years (range 5.7 to 9.1 years) and, for a subgroup of children only, 14 years (range 11 to 15 years) across three publications. The median follow-up was seven years, range six to nine years, in Sillanpaa
2009.

\section{Primary outcomes for review}

All included studies provided data for our primary outcome of recurrent dislocation and used a validated health-related quality of life measure, the Kujala patellofemoral disorders score. Two studies reported the Tegner activity score (Nikku 1997; Sillanpaa 2009). Validated patient-completed outcome measures included the Knee Injury and Osteoarthritis Outcome Score (KOOS) (Christiansen 2008), the Lysholm knee score (Nikku 1997) and the Hughston VAS knee score (Nikku 1997).

\section{Secondary outcomes for review}

Other knee function and activities were reported in two studies (Nikku 1997; Sillanpaa 2009); return to former activities in one study (Sillanpaa 2009); knee pain using a visual analogue scale (VAS) in two studies (Nikku 1997; Sillanpaa 2009); and adverse events relating to treatment in one study (Nikku 1997). Participant satisfaction was reported in two studies (Nikku 1997; Petri 2013). There was variation in the definitions used for 'instability'. Nikku 1997, Petri 2013 and Sillanpaa 2009 included both dislocation and subluxation data. Christiansen 2008 did not report data on subluxation. Bitar 2012 and Nikku 1997 reported also the frequency of recurrent patellar and subluxation events. Two studies (Nikku 1997; Sillanpaa 2009) reported the number of participants in each group who underwent subsequent surgery.

\section{Excluded studies}

We excluded eight studies from the review as they were not randomised or quasi-randomised trials (see Characteristics of excluded studies).

\section{Risk of bias in included studies}

Our judgements of the risk of bias in the six included trials are summarised in the 'Risk of bias' graph (Figure 2) and the 'Risk of bias' summary (Figure 3). 
Figure 2. 'Risk of bias' graph: review authors' judgements about each risk of bias item presented as percentages across all included studies

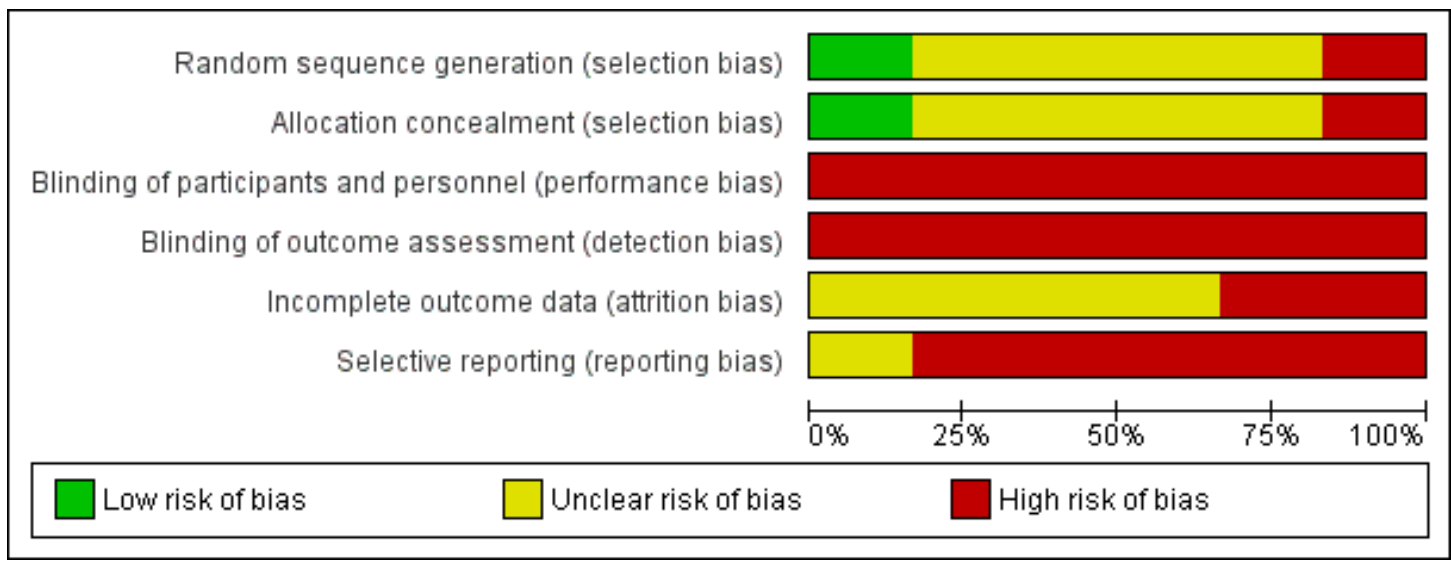


Figure 3. 'Risk of bias' summary: Review authors' judgements about each risk of bias item for each included study

\begin{tabular}{|c|c|c|c|c|c|c|}
\hline & 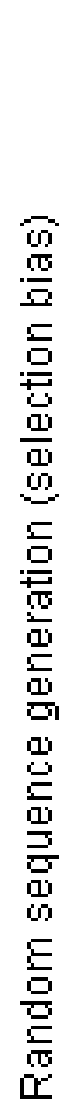 & 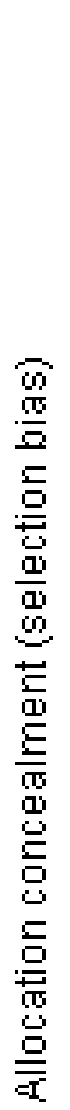 & 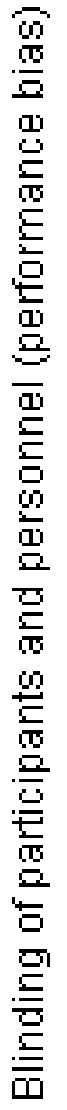 & 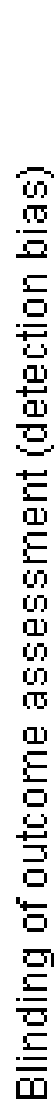 & 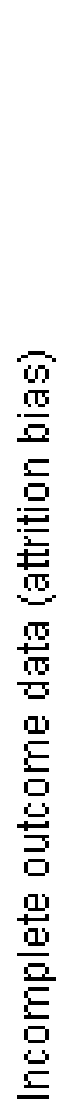 & 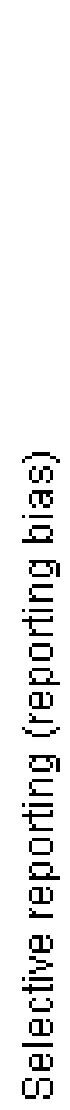 \\
\hline \multicolumn{7}{|l|}{ Bitar 2012} \\
\hline \multicolumn{7}{|l|}{ Camanho 2009} \\
\hline \multicolumn{7}{|l|}{ Christiansen 2008} \\
\hline Nikku 1997 & & & & & $?$ & $?$ \\
\hline Petri 2013 & & & & & $?$ & \\
\hline Sillanpaa 2009 & $?$ & $?$ & & & $?$ & \\
\hline
\end{tabular}




\section{Allocation}

We judged that only Petri 2013 was at low risk of selection bias; this reflected the use of a computer-generated randomisation sequence and sealed envelopes in this trial. The quasi-randomised trial of Nikku 1997, which allocated treatment according to year of birth, was at 'high risk' of selection bias relating to inadequate sequence generation and lack of allocation concealment. The other four trials were at unclear risk of selection bias, which reflected the inadequate information on randomisation methods in these trials. Bitar 2012 and Camanho 2009 probably used the same method involving drawing of a slip of paper specifying the treatment. Christiansen 2008 referred to the random drawing of envelopes and Sillanpaa 2009 to sealed envelopes.

\section{Blinding}

None of the studies blinded their assessors to treatment allocation. Due to the design of these studies, and the topic under investigation, it would have been very difficult, if not impossible, to blind treating clinicians to treatment allocation, or participants to their allocation intervention. All studies were assessed as being at 'high' risk of bias relating to lack of blinding.

\section{Incomplete outcome data}

Small losses to follow-up were reported in five studies (Bitar 2012; Christiansen 2008; Petri 2013; Nikku 1997; Sillanpaa 2009). There were no losses reported in Camanho 2009; but the inclusion criteria indicate a possibility that some may have occurred. Where reported, the numbers of participants lost to follow-up were similar between the groups. Only Petri 2013 and Sillanpaa 2009 reported reasons for their missing participants. Only Bitar 2012 confirmed that the data were analysed according to intention-totreat principles. Follow-up was at set times in Christiansen 2008 and Petri 2013, but spanned two or more years at follow-up in Bitar 2012; Camanho 2009; Nikku 1997; Sillanpaa 2009. There was a 10-month difference between mean length of follow-up for surgical (38 months) and non-surgical (48 months) in Bitar 2012 and a four-month difference (mean 40.4 months in the surgery group versus 36.3 months in the non-surgical group) in Camanho 2009. We judged these two trials top be at high risk of attrition bias and the other four trials at unclear risk.

\section{Selective reporting}

No protocols or prospective trial registration documents were available for any of the six trials. Although all of the planned outcomes defined in the methods section were reported in the results sections of these trials, we judged that the five trials not reporting on adverse effects of surgery were at high risk of selective reporting bias. Since Nikku 1997 did report on adverse effects of surgery, we judged this to be at unclear risk of selective reporting bias.

\section{Effects of interventions}

See: Summary of findings for the main comparison Surgical compared with non-surgical treatment for patellar dislocation The six included trials compared surgical versus non-surgical intervention in people with primary dislocation.

\section{Primary outcomes}

\section{Recurrent dislocation}

All six studies reported the frequency of recurrent dislocation after surgery compared with non-surgical interventions. Data for this outcome are presented for two to five, six to nine and 14 years follow-up periods; see Analysis 1.1; Figure 4. 
Figure 4. Forest plot of comparison I. Surgical versus non-surgical management. Outcome: I.I Number of participants sustaining recurrent patellar dislocation

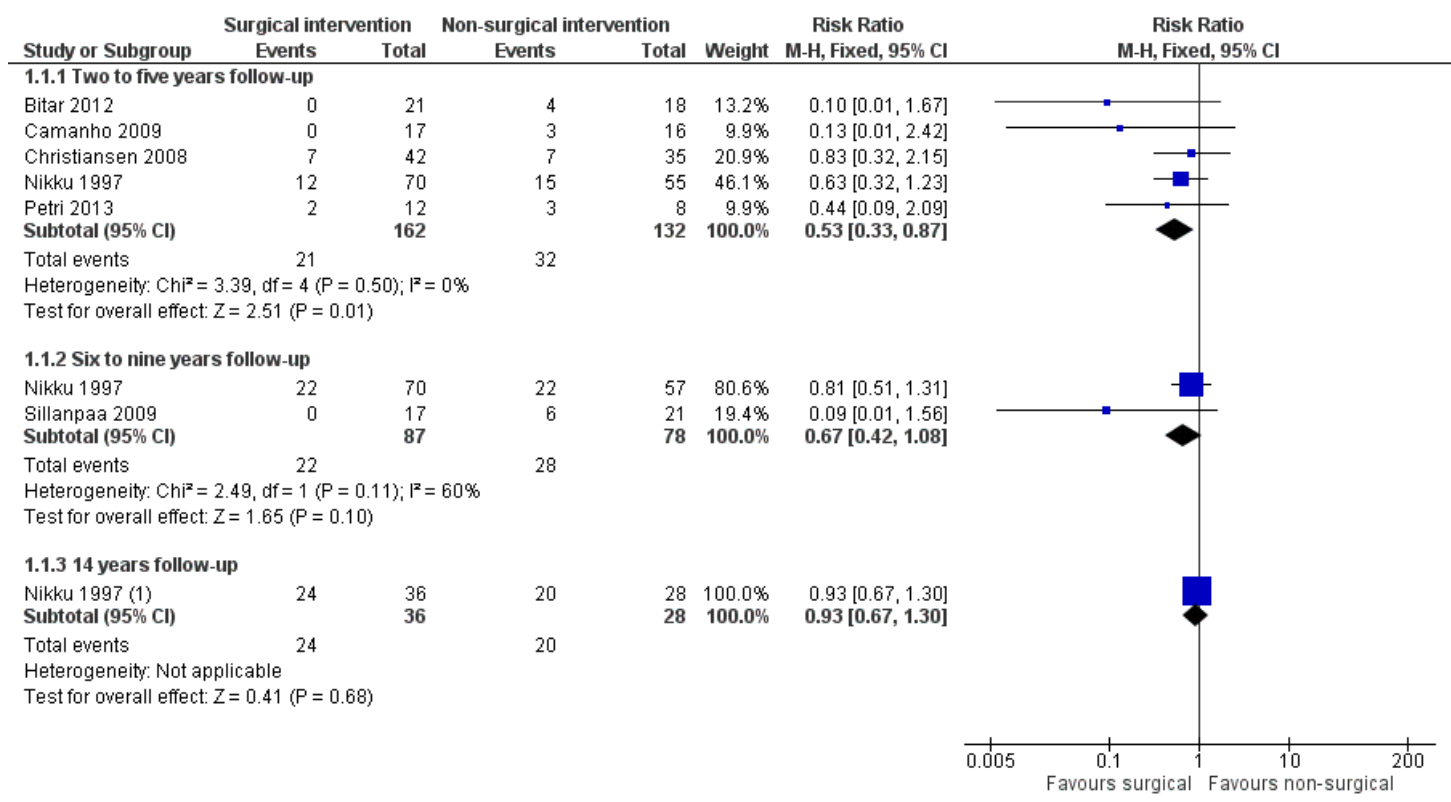

Footnotes

(1) Children only subgroup

Pooled data from five trials (Bitar 2012; Camanho 2009; Christiansen 2008; Nikku 1997; Petri 2013) showed a smaller incidence of recurrent dislocation at two to five years follow-up in the surgical group (21/162 versus 32/132; risk ratio (RR) 0.53 favouring surgery, $95 \%$ confidence interval (CI) 0.33 to 0.87 ; five studies, 294 participants; Analysis 1.1; Figure 4). The incidence of recurrent dislocation was also lower in the surgical group at six to nine years follow-up (22/87 versus $28 / 78$, RR 0.67 favouring surgery, $95 \%$ CI 0.42 to 1.08 ; two studies, 165 participants). Data for a subgroup involving children only of Nikku 1997, reported in Palmu 2008, showed little difference between surgical and nonsurgical groups at 14 years follow-up (24/36 versus 20/28; RR 0.93 favouring surgery, $95 \%$ CI 0.67 to $1.30 ; 64$ participants).

\section{Sensitivity analysis}

The results of the only trial at low risk of selection bias (Petri 2013) were inconclusive at two years (2/12 versus 3/8; RR 0.44 favouring surgery, $95 \%$ CI 0.09 to $2.09 ; 20$ participants).

\section{Validated patient-rated knee and physical function scores for patellar dislocation outcomes}

Two trials (Nikku 1997; Sillanpaa 2009) reported the Tegner activity score (0 to 10: best function); see Analysis 1.2. Nikku 1997 found lower Tegner scores in the surgical group at two years follow-up (mean difference (MD) -0.60 favouring non-surgical treat- ment, $95 \%$ CI -1.28 to 0.08 ; 125 participants); at six to nine years follow-up (surgical versus non-surgical treatment: medians (interquartile range) 4 (3 to 5) versus 5 ( 4 to 6 ); reported $P$ value $=0.03$ ); and, for a subgroup including children only, at 14 years (MD -1.60 favouring non-surgical treatment, 95\% CI -2.44 to $0.76 ; 64$ participants). There was no difference in the mean Tegner activity scores at six to nine years follow-up in Sillanpaa 2009: MD 0.00 , 95\% CI -1.15 to 1.15 ; 40 participants).

The Knee Injury and Osteoarthritis Outcome Score (KOOS) was assessed by Christiansen 2008, who found small non-significant differences between surgical and non-surgical intervention groups at two years in the KOOS symptoms, pain, activities of daily living $(\mathrm{ADL})$, sports and recreation or quality of life subsections $(\mathrm{P}>$ 0.05). The results from this analysis are presented in Analysis 1.3. Nikku 1997 found no significant difference between the two groups in the Lysholm knee score (0 to 100: best outcome) at a mean of two years: $\mathrm{MD}-1.00$ favouring non-surgical treatment, 95\% CI -4.63 to 2.63; 125 participants; see Analysis 1.4 .

Nikku 1997 found lower Hughston VAS (visual analogue scale) patellofemoral scores (28 to 100: best outcome) in the surgical group at a mean of two years (MD -2.80 favouring non-surgical treatment, $95 \%$ CI -6.70 to $1.10 ; 125$ participants); at a mean of seven years (surgical versus non-surgical treatment: medians (interquartile range) 89 (74 to 95) versus 94 (84 to 96); reported 
P value $=0.08)$; and, for a subgroup including children only, at 14 years (MD -7.00 favouring non-surgical treatment, 95\% CI 13.95 to -0.05 ; 64 participants); see Analysis 1.5.

\section{Specific tools for assessing patellar disorders: Kujala patellofemoral disorders score}

The Kujala patellofemoral disorders score (0 to 100 : best outcome) was evaluated in all six studies. Data for this outcome are presented two to five, six to nine and 14 years follow-up periods; see Analysis 1.6; Figure 5). Pooled data from four trials showed higher scores in the surgical group at two to five years (MD 13.93 favouring surgery, $95 \%$ CI 5.33 to $22.53 ; 171$ participants). Although based on data for people with anterior knee pain, this result includes a minimal clinically important difference (MCID) of 10 points (Bennell 2000; Crossley 2004). However, the opposite direction of effect was found at six to nine years (MD -3.25 favouring nonsurgical treatment, $95 \%$ CI -10.61 to 4.11 , two studies, 167 participants) and, for a subgroup including children only, at 14 years (MD -1.00 favouring non-surgical treatment, 95\% CI -8.60 to 6.60 , subgroup of one study, 64 participants). Although the absolute difference of the lower $95 \%$ CI was marginally greater than the MCID for the six to nine years result, in essence neither of the later follow-up results included a clinically significant effect.

Figure 5. Forest plot of comparison: I Surgical versus non-surgical management, outcome: I.6 Kujala patellofemoral disorders score (0 to 100: best outcome)

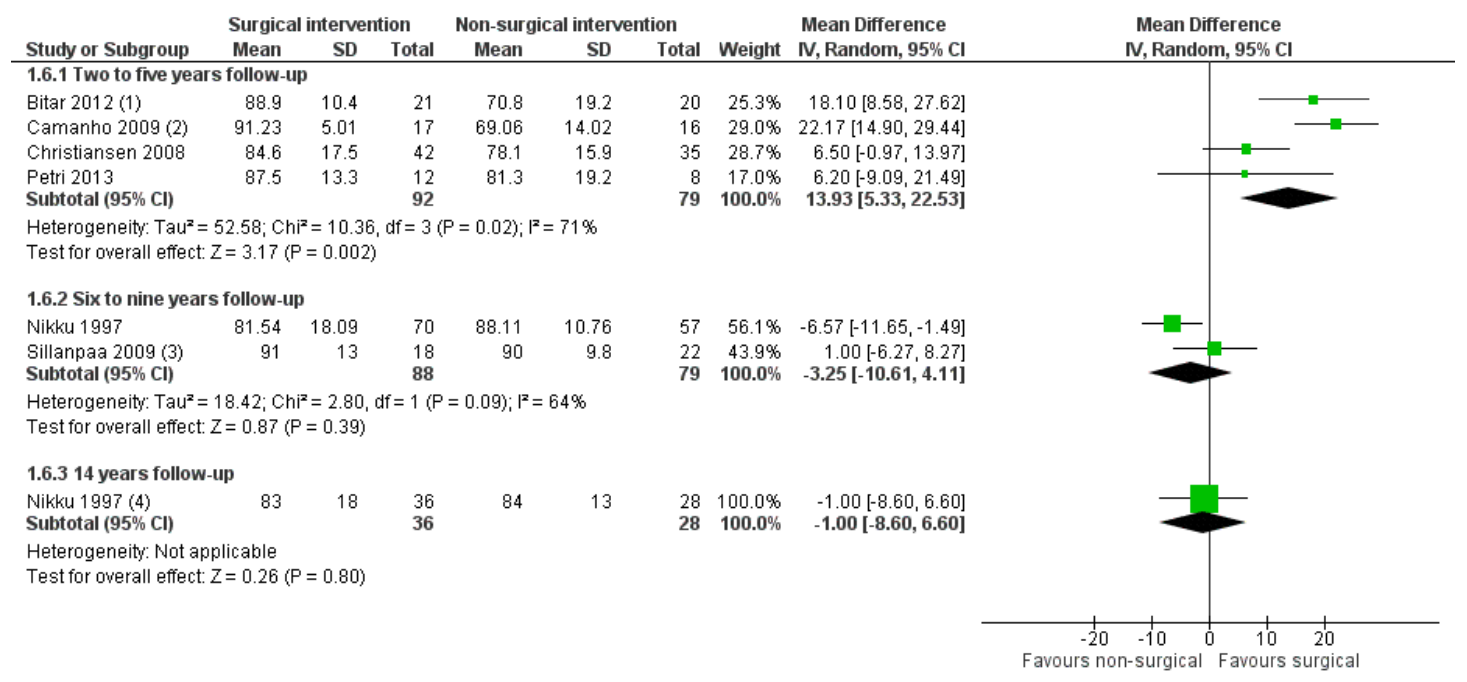

Footnotes

(1) 2 participants in non-surgical group had bilateral involvement - data for 20 knees of 18 participants (2) Standard deviation values obtained from authors in 2010 (Bitar A, Personal communication) (3) Standard deviation values obtained from authors in 2010 (Sillanpaa P, personal communication) (4) Children only subgroup

\section{Sensitivity analysis}

The results of the only trial at low risk of selection bias (Petri 2013) were inconclusive at two years (MD 6.20 favouring surgery, 95\% CI -9.09 to $21.49 ; 20$ participants).

\section{Subgroup analysis: male versus female participants}

Bitar 2012 provided separate Kujala patellofemoral disorders score data for males and females at two to five years. Analysis 1.7 shows no significant differences in the effect sizes between the two sub- groups (test for subgroup differences: $\mathrm{Chi}^{2}=0.11, \mathrm{df}=1(\mathrm{P}=$ $\left.0.74), \mathrm{I}^{2}=0 \%\right)$.

\section{Secondary outcomes}

\section{Other knee function and activity scores}

Nikku 1997 conducted performance tests at a mean of two-years consisting of timed 'figure-of-eight' running, one leg hop distance 
and maximum number of squat downs in one minute. They reported significantly better squat results $(\mathrm{P}=0.03)$ and superior timed 'figure-of-eight' run performance $(\mathrm{P}=0.004)$ in the nonsurgical group compared with the surgery group. They reported no significant difference in one-leg hop quotient between the interventions ( $\mathrm{P}=0.8$ ). Patient-reported outcomes of activity level were evaluated in Sillanpaa 2009. They reported that there was no statistically significant difference between group differences in the subjective assessment of functional knee limitations for stairs, running and squatting $(\mathrm{P}>0.05)$.

\section{Return to former activities: work and sports}

Sillanpaa 2009 reported little between-group difference in the frequency of participants who regained the same activity level as before their dislocation (13/17 versus 15/21; RR 1.07 favouring surgery, $95 \%$ CI 0.73 to 1.56 ; 38 participants; Analysis 1.8).

\section{Knee pain during activity or at rest}

Two studies assessed knee pain using a VAS, one at a mean of two years (Nikku 1997) and the other at six to nine years (Sillanpaa 2009); see Analysis 1.9. Neither found a significant difference between treatment groups. The results for Nikku 1997 were: MD 0.20 favouring non-surgical treatment, $95 \% \mathrm{CI}-0.29$ to 0.67 , 125 participants). The results for Sillanpaa 2009 were: MD 0.50 favouring non-surgical treatment, $95 \% \mathrm{CI}-0.28$ to $1.28,38$ participants).

\section{Complications/adverse events of interventions}

Only Nikku 1997 reported on adverse effects of treatment; restricting their account to 'major' complications that occurred in four participants of the surgical group. These were: paresis of the sciatic nerve, possibly due to tourniquet compression and resulting in severe permanent disability; a deep wound infection and bacterial arthritis, which resolved with revision surgery and antibiotic therapy; a superficial wound infection; and a burn injury on the insensible anterior aspect of the knee.

\section{Range of knee motion}

Range of knee motion was assessed in one study (Sillanpaa 2009). There was no statistically significant difference in total knee range of motion of the affected knee between surgical (median 138 degrees) and non-surgical (median 140 degrees) interventions at the six to nine years follow-up $(\mathrm{P}>0.05)$.

\section{Patient-reported satisfaction}

Patient satisfaction was assessed in two studies (Nikku 1997; Petri 2013); see Analysis 1.10). Pooled data at a mean of two years follow-up showed little difference between the groups in 'good or excellent' ratings of treatment outcome by participants (57/81 versus 43/63; RR 1.03 favouring surgery, $95 \%$ CI 0.83 to 1.29 ; two studies, 144 participants). Nikku 1997 found higher incidences of satisfaction (good or excellent ratings) in the non-surgical group at a mean of seven years ( $47 / 70$ versus 46/57; RR 0.83 favouring non-surgical treatment, $95 \%$ CI 0.68 to $1.02 ; 127$ participants) and, for a subgroup involving children only, at the 14 years (21/ 32 versus 21/28; RR 0.88 favouring non-surgical treatment, $95 \%$ CI 0.63 to 1.22 ; one study, 60 participants).

\section{Patient-reported instability symptoms}

\section{Patellar subluxation}

The numbers of participants reporting an episode or episodes of patellar subluxation during follow-up were recorded in five studies (Bitar 2012; Camanho 2009; Nikku 1997; Petri 2013; Sillanpaa 2009). Data for this outcome are presented at two to five and six to nine years follow-up periods. The incidence of participants reporting patellar subluxation was lower in the surgical group at two to five years follow-up (16/119 versus 21/97; RR 0.61 favouring surgery, $95 \%$ CI 0.35 to 1.07 ; four studies, 216 participants; Analysis 1.11). This pooled analysis exhibited some heterogeneity $\left(\mathrm{Chi}^{2}=5.03, \mathrm{df}=3(\mathrm{P}=0.17), \mathrm{I}^{2}=40 \%\right)$. However, there was minimal difference between groups found at six to nine years follow-up (25/87 versus 22/78; RR 0.98, 95\% CI 0.60 to 1.58 ; two studies, 165 participants; Analysis 1.11).

\section{Any instability episode}

The number of participants in each group suffering episodes of instability (dislocation, subluxation or both) was reported in four studies (Bitar 2012; Camanho 2009; Nikku 1997; Sillanpaa 2009). The incidence of participants with instability was lower in the surgical group at two to five years follow-up (18/108 versus $34 / 89$; RR 0.44 favouring surgery, $95 \%$ CI 0.27 to 0.72 ; three studies, 197 participants) and at six to nine years follow-up (47/87 versus 50/78; RR 0.80 favouring surgery, $95 \%$ CI 0.62 to 1.03 ; two studies, 165 participants); see Analysis 1.12). Both pooled analyses exhibited significant heterogeneity: $\mathrm{Chi}^{2}=6.95, \mathrm{df}=2(\mathrm{P}$ $=0.03), \mathrm{I}^{2}=71 \%$; and $\left.\mathrm{Chi}^{2}=3.94, \mathrm{df}=1(\mathrm{P}=0.05), \mathrm{I}^{2}=75 \%\right)$.

\section{Subsequent requirement for surgery}

Two studies (Nikku 1997; Sillanpaa 2009) reported the number of participants in each group who had undergone subsequent surgical intervention; see Analysis 1.13. Subsequent surgery was marginally higher in the surgery group at two years (20 to 45 months) in Nikku 1997 (12/70 versus 9/55; RR 1.05 favouring non-surgical treatment, $95 \%$ CI 0.48 to $2.31 ; 125$ participants); at six to nine years (20/87 versus 16/78; RR 1.06 favouring non-surgical treatment, $95 \%$ CI 0.59 to 1.89 ; two studies, 165 participants); 
and, for a children-only subgroup of Nikku 1997, at 14 years (16/ 36 versus $11 / 28$; RR 1.13 , 95\% CI 0.63 to 2.04; one study, 64 participants). Several participants in Nikku 1997 had more than one operation.

\section{ISCUSSION}

\section{Summary of main results}

The findings of this review are based on six studies involving 344 participants with primary patellar dislocation. The mean ages in the individual studies ranged from 19.3 to 25.7 years, with four studies including children, mainly adolescents, as well as adults. Based on our assessment of the evidence using the GRADE approach, we rated the quality of evidence for each reported outcome as 'very low' (see Summary of findings for the main comparison); this means that we are very uncertain about the estimates and that further research is very likely to have an important impact on the estimates of effect.

The strongest evidence was for recurrent patellar dislocation, which was consistently less common in the surgical groups of all six trials. Pooled two to five years follow-up data from five trials (294 participants) indicated that, based on an assumed risk of recurrent dislocation in 222 people per 1000 in the non-surgical group, 104 fewer ( $95 \%$ CI 149 fewer to 28 fewer) people per 1000 had recurrent dislocation as a result of surgery. Pooled six to nine years follow-up data from two trials (165 participants) indicated that, based on an assumed risk of recurrent dislocation in 336 people per 1000 in the non-surgical group, 110 fewer (95\% CI 195 fewer to 27 more) people per 1000 had recurrent dislocation as a result of surgery.

Very low quality evidence was available for presentation in forest plots from single trials only for four validated patient-rated knee and physical function scores for patellar dislocation: the Tegner activity scale, KOOS, Lysholm and Hughston VAS score. The mean Tegner scores were identical in two treatment groups at six to nine years follow-up in one trial but reported to be higher (indicating a better outcome) in the non-surgical group of a second trial. The confidence intervals of the results for the KOOS, Lysholm and Hughston VAS scores crossed over the line of no effect and probably did not include a clinically relevant effect. The results for the Kujala patellofemoral disorders score (0 to 100: best outcome) differed in direction of effect at two to five years follow-up, which favoured the surgery group (MD 13.93 points higher, 95\% CI 5.33 points higher to 22.53 points higher; four studies, 171 participants) and the six to nine years follow-up, which favoured the non-surgical treatment group (MD 3.25 points lower, 95\% CI 10.61 points lower to 4.11 points higher; two studies, 167 participants). The confidence interval for this outcome from the first time period included the minimal clinically important difference of 10 (estimated for people with anterior knee pain) thus pointing to the possibility of a clinically important effect. Adverse effects of treatment were reported in one trial only; all four major complications were attributed to the surgical treatment group. Slightly more people in the surgery group had subsequent surgery six to nine years after their primary dislocation. Pooled six to nine years follow-up data from two trials (165 participants) indicated that, based on an assumed risk of subsequent surgery in 186 people per 1000 in the non-surgical group, 11 more (95\% CI 76 fewer to 171 more) people per 1000 had subsequent surgery after primary surgery.

\section{Overall completeness and applicability of evidence}

The objective of the review, to assess the benefits and harms of surgical compared with non-surgical interventions for treating people with primary or recurrent patellar dislocation, has been met in part. Our findings are relevant to the management of people who seek treatment following a first-time or primary lateral patellar dislocation. However, there are no randomised controlled trials (RCTs) that have assessed the outcomes of surgical or non-surgical interventions following recurrent or secondary patellar dislocation. Only one study (Nikku 1997) measured and reported the frequency of adverse events. Furthermore, only Nikku 1997 presented results for a children-only subgroup, that consisted mainly of adolescent participants with primary patellar dislocation. The findings of this review should therefore be interpreted with some caution for patients under 16 years of age, and should not be used to justify the treatment of those people who are managed following recurrent lateral patellar dislocation. Furthermore, only Nikku 1997 reported long-term outcomes, albeit for a subgroup of their patellar dislocation cohort. It therefore remains uncertain what the long-term outcomes are for this population. Nikku 1997 noted that both treatment groups reported high recurrent dislocation rates but that functionally, children had good outcomes, and were able to perform all their activities of daily living (ADL), irrespective of recurrent patellar instability and dislocation events. Finally, no studies assessed whether the presence or absence of generalised joint or specific patellar hypermobility was an important variable on outcome.

The data were insufficient to perform pre-specified subgroup analyses exploring whether the treatment effect differed importantly according to key patient characteristics. One study, however, permitted a subgroup analysis of Kujala patellofemoral disorders score between male and female participants (Bitar 2012). This indicated no statistically significant difference in outcome between treatments dependent on gender, thus providing some very limited indication that the results of this review can be applied to both sexes. A number of different surgical and rehabilitative interventions were used in the included studies. It was not possible to determine the relative efficacy of individual interventions such as medial patellofemoral ligament (MPFL) repair versus reconstruction. 
This indirect comparison would be a valuable subgroup analysis as further data become available in a future update. In addition, there was a degree of clinical heterogeneity amongst participants. For instance, some individuals suffering patellar dislocation may have had predisposing factors (e.g. family history, particular anatomical morphology of the patellofemoral joint, soft tissue integrity or hypermobility). Furthermore, some participants suffered complications resulting from their patellar dislocation such as separation of osteochondral fragments into the knee joint. Although some studies reported these factors (Nikku 1997; Sillanpaa 2009), the included studies were uneven in the description of anatomical pathology present in their participants, the diagnostic procedures used to investigate them or the rationale for choice of surgical technique.

As acknowledged in the Description of the condition, the aetiology of patellar dislocation is multifactorial. Consequently, there can be a degree of heterogeneity with respect to clinical presentation contributing to, or causing the dislocation. As a result, the need for surgery may be slightly different between individuals. This may be regarded as a limitation. However, there was no evidence from the original papers of a significant level of clinical heterogeneity to negate appropriate meta-analyses.

The non-surgical management reported in the included studies were generally poorly described. Whilst most studies appropriately reported the method and duration of immobilisation, all included studies poorly described their rehabilitation regimens such as type of exercises prescribed or the frequency, duration or intensity. This has been previously acknowledged as a widespread limitation within the patellar instability literature (Smith 2010). Consequently, it was not possible to assess effectively clinical heterogeneity in the non-surgical management of participants. It should be noted that all 'non-surgical' group participants had had diagnostic arthroscopy prior to randomisation in Christiansen 2008 and all participants received knee aspiration to relieve pain in Sillanpaa 2009.

\section{Quality of the evidence}

All six trials had serious methodological weaknesses, in particular resulting from lack of blinding, that placed them all at high risk of performance and detection bias. Only Nikku 1997 included more than 100 participants; the other trials were small and insufficiently powered. The dominance of Nikku 1997 is evident in all the analyses, which is of particular note because it was quasi-randomised and thus at high risk of selection bias. There also may have been a risk of publication and other reporting bias due to the small number of small studies included (Song 2010). Where reported, there were few losses to follow-up but differences in the follow-up times between the treatment groups in two trials (Bitar 2012; Camanho 2009) meant these were likely to be at high risk of attrition bias. Only Christiansen 2008 and Petri 2013 had set follow-up times (final follow-up was two years in both trials), whereas, the period of follow-up spanned three years in Nikku 1997 (intermediate follow-up) and Sillanpaa 2009. Another limitation is that the bulk of the evidence pertained to two to five years follow-up.

There was clinical heterogeneity amongst the individual included studies, including in the surgical methods used. For the primary outcome of recurrent episodes of dislocation, it is notable that the included studies fell into two groups. In three trials, no recurrent dislocation occurred in the surgical group (Bitar 2012; Camanho 2009; Sillanpaa 2009), whereas recurrent dislocation occurred in the surgical groups of the other three trials. We cannot detect an obvious clinical reason for this difference, which may anyway reflect in part the small sample sizes of these trials.

We assessed the quality of the evidence as 'very low' for all outcomes. Two generalities applied. For all outcomes that included evidence from Nikku 1997, we downgraded the evidence two levels for serious limitations in study design. For all outcomes with evidence from Nikku 1997 only, we further downgraded the evidence one level for serious imprecision. For all outcomes with evidence from a single trial that was not Nikku 1997, we downgraded the evidence one level for limitations in study design and two levels for serious imprecision. As the evidence was dominated by Nikku 1997 for all outcomes with pooled data, we downgraded the evidence for these two levels for serious limitations in study design. As there were often two or more reasons for downgrading of these outcomes, we have selected the main one in our account below. We downgraded the evidence for recurrent dislocation (two to five years follow-up and six to nine years follow-up) one further level for imprecision. We downgraded the evidence for four outcomes (Kujala patellofemoral disorders score results, recurrent subluxation, any episode of instability and subsequent surgery) one further level for inconsistency. We downgraded the evidence for patient satisfaction one further level for indirectness. This grading means that we are very uncertain about the estimates of effect (Summary of findings for the main comparison).

\section{Potential biases in the review process}

We consider that our search strategy was comprehensive and believe, but cannot be completely sure, that we have identified all relevant published trials that met the inclusion criteria. We cannot, of course, rule out the failure to identify trials published in non-indexed journals or unpublished trials.

While we have consistently presented recurrent dislocation as our primary outcome, some have questioned whether it is correct to separate dislocations from subluxations and from episodes of instability rather than presenting these together as the primary measure of treatment success or failure. We consider that our approach continues to be correct and that our decision to separate patellar dislocation from subluxation and general perceived instability symptoms is justified through the distinction between mechanical and functional-derived instability (Donell 2006a). When setting up our protocol we considered that mechanical instability is re- 
lated to the success of a surgical procedure, whereas functional instability is related to neuro-muscular control and therefore either the rehabilitation of an individual post-operatively or as part of a non-surgical rehabilitation programme. Thus, dislocation, unlike subluxation or episodes of instability, reflects failure of surgery. In contrast subluxation and instability are more reflective of post-operative complications or failings in rehabilitation. Based on this, we felt that it was important to analyse these separately. This approach also reflects the current evidence base, which has presented these data separately.

\section{Agreements and disagreements with other studies or reviews}

Systematic reviews performed by the authors of the outcomes of MPFL repair (Smith 2007), trochleoplasty (Smith 2008) and nonoperative rehabilitation interventions (Smith 2010) have been reported. No relevant randomised trials were identified by these reviews. We also conducted a meta-analysis including five randomised and six non-randomised controlled trials assessing surgical compared to non-surgical interventions for patients with patellar dislocation and reported similar concerns regarding the methodological quality of the current evidence base (Smith 2011). In Smith 2011, we reported a statistically significant difference between interventions for the outcomes of frequency of recurrent dislocation, development of osteoarthritis and Hughston VAS patellofemoral score.

The Stefancin 2007 systematic review compared surgical with nonsurgical management of primary traumatic patellar dislocation. They included 70 studies, all but one of which were non-randomised, published up to the end of 2006. Due to these different eligibility and search criteria, the only paper included in both our review and Stefancin 2007 is Nikku 1997. The findings between this Cochrane review and the Stefancin 2007 paper have some differences. Stefancin 2007 recommended that initial management of primary patellar dislocation should be non-surgical, except in cases where there was an osteochondral fragment evident, a chondral injury or a large medial patellar stabiliser defect as detected by computed tomography (CT) and/or magnetic resonance imaging (MRI) findings. This recommendation was based on the conclusion that the outcomes of surgical and non-surgical interventions were not dissimilar, but that any major complications were in the surgically managed groups. However, in respect of recurrent patellar dislocation, our review would suggest that surgical consideration may be an appropriate strategy for the management of this population, to reduce the risks of recurrent dislocation and instability.

The Frosch 2011 and Sillanpää 2012 reviews also reported limited difference in clinical outcomes between surgical and non-surgical interventions but suggested that decision-making on treatment options should include an assessment of anatomical risk factors for recurrent dislocation. They suggested that people with a nor- mal or minor dysplastic patellofemoral joint may be more suitable for non-surgical treatment, whilst those with a higher grade of trochlear dysplasia or other significant morphological abnormalities may benefit from surgical treatment. Since the current evidence base has not provided sufficient information on morphological features, it is not possible to perform a subgroup analysis to test these hypotheses. Similar findings were reported by Baier 2011, Sillanpää 2012 and Tsai 2012.

It was not possible to compare the findings of this review with a recent systematic review (Saccomanno 2012), given that this has only been presented as a conference abstract. The publication stage of this review will be determined and appropriately incorporated in subsequent review updates.

\section{A U THORS' CONCLUSIONS}

\section{Implications for practice}

No randomised or quasi-randomised controlled trials have assessed the outcomes of surgical compared with non-surgical treatments in people who seek treatment following a secondary or recurrent patellar dislocation.

There is very low quality evidence to support surgical over nonsurgical management of primary patellar dislocation in the short term (two to five years follow-up) based on a finding of a significantly lower risk of recurrent dislocation and superior scores for a patient-reported outcome measure for patellar disorders. However, due to the very low quality and incompleteness of the evidence, this finding must be viewed with caution until a stronger evidence base is established. This should be the case unless there are specific indications for a surgical intervention; such indications include an osteochondral fracture or other intra-articular disorder within the knee joint, or demonstrated evidence of a major tear of the medial soft tissues stabilising the patella.

\section{Implications for research}

The evidence from the currently published trials is 'very low' quality evidence, which means that we are very uncertain about the estimates and that further research is very likely to have an important impact on the estimates of effect.

Based on the incidence of recurrent dislocation in the studies included in this review, a case could be made for a multi-centre randomised trial managed from a clinical research centre, enrolling in excess of 600 participants, conducted and reported to the standards of the CONSORT (Consolidated Standards of Reporting Trials) statement (CONSORT 2010). We suggest that before such a trial is conducted, expert consensus be achieved on the standards for future research in this area. This might include clearer definition of both surgical and non-surgical interventions, and a suite 
of "standard" outcomes that should be reported. These might include recurrent dislocation, recurrent subluxation, recurrent instability episodes, validated functional and quality of life scores. Follow-up should be assessed at set time points; we suggest two, five and 10 yearS follow-up would be suitable. As individuals with patellar instability may have multiple episodes, recording both the number of participants sustaining an event and the number of events in each group to allow calculation of both risk rate and rate ratio would be desirable. Key anatomical or pathological factors particularly relevant to the natural history of patellar instability, and thus to the choice of intervention, should also be recorded. Such a consensus would inform the design and conduct of a large study of management of primary patellar instability, and would be useful also in research evaluating the place of surgery in the management of recurrent dislocation.

\section{ACKNOWLEDGEMENTS}

We thank Helen Handoll, Paul Jenkins and Haris Vasiliadis for their feedback on this review update. We thank Laura MacDonald for editorial support and Joanne Elliott for her help with the database searches.

We thank Helen Handoll, Paul Jenkins and Jane Mackintosh for their constructive comments on the protocol and original review; and Lindsey Elstub for editorial support. We also acknowledge Joanne Elliott for her help in the development of the search strategies. We would particularly like to thank Professor William Gillespie, for his guidance on the first version of the review.

We would like to thank Alexandre Bitar, University of São Paulo, Brazil, Peter Balcarek, University Medical Center Göttingen, Germany, Marco Demange, University of São Paulo, Brazil, Risto Nikku, Helsinki University Central Hospital, Finland, Martin Lind, Aarhus University Hospital, Denmark, Maximilian Petri, Medizinische Hochschule, Hannover, Germany and Petri Sillanpaa, Central Military Hospital, Helsinki, Finland, for providing additional data used as part of the meta-analysis and for screening the provisional list of included studies.

We would also like to thank the library staff at the Norfolk and Norwich University Hospital's Sir Thomas Browne Library and University of East Anglia's Library who assisted in the gathering of papers required for this review.

This project was supported by the National Institute for Health Research via Cochrane Infrastructure funding to the Cochrane Bone, Joint and Muscle Trauma Group. The views and opinions expressed therein are those of the authors and do not necessarily reflect those of the Systematic Reviews Programme, NIHR, NHS or the Department of Health.

\section{RE F E R E N C E S}

\section{References to studies included in this review}

\section{Bitar 2012 \{published data only\}}

Bitar A. Personal communication 25 October 2013.

* Bitar AC, Demange MK, D’Elia CO, Camanho GL. Traumatic patellar dislocation: nonoperative treatment compared with MPFL reconstruction using patellar tendon. American Journal of Sports Medicine 2012;40(1):114-22.

\section{Camanho 2009 \{published data only\}}

Bitar A. Personal communication 19 January 2010.

* Camanho GL, Viegas Ade C, Bitar AC, Demange MK, Hernandez AJ. Conservative versus surgical treatment for repair of the medial patellofemoral ligament in acute dislocations of the patella. Arthroscopy 2009;25(6):620-5.

Christiansen 2008 \{published data only\}

* Christiansen SE, Jakobsen B, Lund B, Lind M. Isolated repair of the medial patellofemoral ligament in primary dislocation of the patella: a prospective randomized study. Arthroscopy 2008;24(8):881-7. [MEDLINE: 18657736]

Lind M. Personal communication 22 January 2010.

Nikku 1997 \{published data only\}

Donell S. Personal communication 25 October 2014. Nietosvaara Y, Paukku R, Palmu S, Donell ST. Acute patellar dislocation in children and adolescents. Surgical technique. Journal of Bone \& Joint Surgery - American Volume 2009;91 Suppl 2 Pt 1:139-45.

Nikku R, Nietosvaara Y, Aalto K, Kallio PE. Operative treatment of primary patellar dislocation does not improve medium-term outcome. A 7-year follow-up report and risk analysis of 127 randomised patients. Acta Orthopaedica 2005;76(5):699-704. [MEDLINE: 16263618]

* Nikku R, Nietosvaara Y, Kallio PE, Aalto K, Michelsson JE. Operative versus closed treatment of primary dislocation of the patella. Similar 2-year results in 125 randomized patients. Acta Orthopaedica Scandinavica 1997;68(5): 419-23. [MEDLINE: 9385238]

Palmu S, Kallio PE, Donell ST, Helenius I, Nietosvaara Y. Acute patellar dislocation in children and adolescents: a randomised clinical trial. Journal of Bone \& Joint Surgery - American Volume 2008;90(3):463-70. [MEDLINE: 18310694]

Pieler-Bruha E. Acute patellar dislocation in children and adolescents: A randomized clinical trial: Commentary. Journal für Mineralstoffwechsel 2008;15(2):96-7.

Petri 2013 \{published data only\}

Balcarek P. Personal communication 27 October 2013. Petri M. Personal communication 25 October 2013. * Petri M, Liodakis E, Hofmeister M, Despang FJ, Maier 
$\mathrm{M}$, Balcarek P, et al. Operative vs conservative treatment of traumatic patellar dislocation: results of a prospective randomized controlled clinical trial. Archives of Orthopaedics and Trauma Surgery 2013;133:209-13.

Sillanpaa 2009 \{published data only\}

Sillanpaa P. Personal communication 18 January 2010.

* Sillanpää PJ, Mattila VM, Mäenpää H, Kiuru M, Visuri T, Pihlajamäki H. Treatment with and without initial stabilizing surgery for primary traumatic patellar dislocation. A prospective randomized study. Journal of Bone \& Joint Surgery - American Volume 2009;91(2): 263-70. [MEDLINE: 19181969]

\section{References to studies excluded from this review}

Apostolovic 2011 \{published data only\}

Apostolovic M, Vukomanovic B, Slavkovic N, Vuckovic V, Vukcevic M, Djuricic G, et al. Acute patellar dislocation in adolescents: operative versus nonoperative treatment. International Orthopaedics 2011;35(10):1483-7. [DOI: 10.1007/s00264-011-1265-z]

Arnbjörnsson 1992 \{published data only\}

Arnbjörnsson A, Egund N, Rydling O, Stockerup R, Ryd L. The natural history of recurrent dislocation of the patella. Long-term results of conservative and operative treatment. Journal of Bone \& Joint Surgery - British Volume 1992;74(1): 140-2. [MEDLINE: 1732244]

Buchner 2005 \{published data only\}

Buchner M, Baudendistel B, Sabo D, Schmitt H. Acute traumatic primary patellar dislocation. Long-term results comparing conservative and surgical treatment. Clinical Journal of Sports Medicine 2005;15(2):62-6. [MEDLINE: 15782048]

Cash 1988 \{published data only\}

Cash JD, Hughston JC. Treatment of acute patellar dislocation. American Journal of Sports Medicine 1988;16 (3):244-9. [MEDLINE: 3381981]

Marcacci 1995 \{published data only\}

Marcacci M, Zaffagnini S, Iacono F, Visani A, Petitto A, Neri NP. Results in the treatment of recurrent dislocation of the patella after 30 years' follow-up. Knee Surgery Sports Traumatology Arthroscopy 1995;3(3):163-6. [MEDLINE: 8821272]

Savarese 1990 \{published data only\}

Savarese A, Lunghi E. Traumatic dislocations of the patella: problems related to treatment. Chirurgia Degli Organi di Movimento 1990;75(1):51-7. [MEDLINE: 2369854]

Sillanpää 2008a \{published data only\}

Sillanpää PJ, Mäenpää HM, Mattila VM, Visuri T, Pihlajamäki H. Arthroscopic surgery for primary traumatic patellar dislocation. A prospective, nonrandomized study comparing patients treated with and without acute arthroscopic stabilization with a median 7-year follow-up. American Journal of Sports Medicine 2008;36(12):2301-9. [MEDLINE: 18762668]
Sillanpää 2008b \{published data only\}

Sillanpää P, Mattila VM, Visuri T, Mäenpää H, Pihlajamäki $\mathrm{H}$. Ligament reconstruction versus distal realignment for patellar dislocation. Clinical Orthopaedics and Related Research 2008;466(6):1475-84. [MEDLINE: 18347890]

\section{References to ongoing studies}

\section{ISRCTN39959729 \{published data only\}}

Janarv P-M. Conservative versus arthroscopic refixation of the medial patellofemoral ligament (MPFL) after traumatic first time dislocation of the patella in children: a prospective randomised study. http://www.controlled-trials.com/ ISRCTN39959729 (accessed October 2013).

\section{Additional references}

Atkin 2000

Atkin DM, Fithian DC, Marangi KS, Stone ML, Dobson $\mathrm{BE}$, Mendelsohn C. Characteristics of patients with primary acute lateral patellar dislocation and their recovery within the first 6 months of injury. American Journal of Sports Medicine 2000;28(4):472-9. [MEDLINE: 10921637]

Baier 2011

Baier C, Springorum HR, Beckmann J, Grifka J, Matussek J. Treatment of patellar instability in children and adolescents. Orthopade 2011;40(10):868-76. [DOI: 10.1007/s00132-011-1775-9]

Beasley 2004 Beasley LS, Vidal AF. Traumatic patellar dislocation in children and adolescents: treatment update and literature review. Current Opinion in Pediatrics 2004;16(1):29-36. [MEDLINE: 14758111]

Bennell 2000

Bennell K, Bartam S, Crossley K, Green S. Outcome measures in patellofemoral pain syndrome: test retest reliability and inter-relationships. Physical Therapy in Sport 2000;1(2):32-41.

Boden 1997

Boden BP, Pearsall AW, Garrett WE Jr, Feagin JA Jr. Patellofemoral instability: Evaluation and management. Journal of the American Academy of Orthopaedic Surgeons 1997;5(1):47-57. [PUBMED: 10797207]

\section{Carter 1964}

Carter C, Wilkinson J. Persistent joint laxity and congenital dislocation of the hip. Journal of Bone and Joint Journal American Edition 1964;46:40-5.

\section{Colvin 2008}

Colvin AC, West RV. Patellar instability. Journal of Bone and Joint Surgery - American Volume 2008;90(12):2751-62. [EMBASE: 2008585666]

Conlan 1993

Conlan T, Garth WP Jr, Lemons JE. Evaluation of the medial soft-tissue restraints of the extensor mechanism of the knee. Journal of Bone and Joint Surgery - American Volume. 1993;75(5):682-93. [MEDLINE: 8501083] 


\section{CONSORT 2010}

Schulz KF, Altman DG, Moher D, CONSORT Group. CONSORT 2010 Statement: Updated guidelines for reporting parallel group randomised trials. Journal of Clnical Epidemiology 2010;63(8):834-40. [MEDLINE: 20346629]

Cosgarea 2002

Cosgarea AJ, Browne JA, Kim TK, McFarland EG. Evaluation and management of the unstable patella. Physician and Sportsmedicine 2002;30(10):33-40. [EMBASE: 2002360234]

\section{Crossley 2004}

Crossley KM, Bennell KL, Cowan SM, Green S. Analysis of outcome measures for persons with patellofemoral pain: which are reliable and valid?. Archives of Physical Medicine and Rehabilitation 2004;85(5):815-22.

\section{Dath 2006}

Dath R, Chakravarthy J, Porter KM. Patella dislocations. Trauma 2006;8(1):5-11. [EMBASE: 2006239083]

\section{Dejour 1994}

Dejour H, Walch G, Nove-Josserand L, Guier C. Factors of patellar instability: an anatomic radiographic study. Knee Surgery, Sports Traumatology, Arthroscopy 1994;2(1):19-26. [MEDLINE: 7584171]

\section{Donell 2006a}

Donell ST. Patellofemoral dysfunction-Extensor mechanisms malalignment. Current Orthopaedics 2006;20 (2):103-11.

\section{Donell 2006b}

Donell ST, Joseph G, Hing CB, Marshall TJ. Modified Dejour trochleoplasty for severe dysplasia: operative technique and early clinical results. Knee 2006;13(4): 266-73. [MEDLINE: 16635572]

\section{Donell 2014}

Donell S. Personal communication 25 October 2014.

\section{Fithian 2004}

Fithian DC, Paxton EW, Stone ML, Silvia P, Davis DK, Elias DA, et al. Epidemiology and natural history of acute patellar dislocation. American Journal of Sports Medicine 2004;32(5):1114-21. [MEDLINE: 15262631]

\section{Flandry 1991}

Flandry F, Hunt JP, Terry GC, Hughston JC. Analysis of subjective knee complaints using visual analog scales. American Journal of Sports Medicine 1991;19(2):112-8. [MEDLINE: 2039061]

\section{Frosch 2011}

Frosch S, Balcarek P, Walde TA, Schüttrumpf JP, Wachowski MM, Ferleman KG, et al. The treatment of patellar dislocation: a systematic review [Die Therapie der Patellaluxation: eine systematische Literaturanalyse]. Zeitschrift fur Orthopadie und Unfallchirurgie 2011;149(6): 630-45. [DOI: 10.1055/s-0030-1250691]

\section{Fukushoma 2004}

Fukushima K, Horaguchi T, Okano T, Yoshimatsu T, Saito A, Ryu J. Patellar dislocation: arthroscopic patellar stabilization with anchor sutures. Arthroscopy 2004;20(7): 761-4. [MEDLINE: 15346119]

\section{Guhan 2009}

Guhan B, Lee AS. Acute repair of medial patellofemoral ligament (abstract). Journal of Bone and Joint Surgery British Volume 2009;91(Suppl 3):413-4.

\section{Hautamaa 1998}

Hautamaa PV, Fithian DC, Kaufman KR, Daniel DM, Pohlmeyer AM. Medial soft tissue restraints in lateral patellar instability and repair. Clinical Orthopaedics and Related Research 1998;(349):174-82. [MEDLINE: 9584380]

\section{Hawkins 1986}

Hawkins RJ, Bell RH, Anisette G. Acute patellar dislocations: The natural history. American Journal of Sports Medicine 1986;14(2):117-20. [MEDLINE: 3717480]

\section{Higgins 2011}

Higgins JPT, Altman DG, Sterne JAC (editors). Chapter 8: Assessing risk of bias in included studies. In: Higgins JPT, Green S (editors). Cochrane Handbook for Systematic Reviews of Interventions Version 5.1.0 (updated March 2011). The Cochrane Collaboration, 2011. Available from www.cochrane-handbook.org.

Hing 2006

Hing CB, Shepstone L, Marshall T, Donell ST. A laterally positioned concave trochlear groove prevents patellar dislocation. Clinical Orthopaedics and Related Research 2006;(447):187-94. [MEDLINE: 16467625]

Hsiao 2010

Hsiao M, Owens BD, Burks R, Sturdivant RX, Cameron $\mathrm{KL}$. Incidence of acute traumatic patellar dislocation among active-duty United States military service members. American Journal of Sports Medicine 2010;38(10): 1997-2004.

\section{Kiviluoto 1986}

Kiviluoto O, Pasila M, Santavirta S. Recurrences after conservative treatment of acute dislocation of the patella. Italian Journal of Sports Traumatology 1986;8(3):159-62. [EMBASE: 1987071200]

\section{Kujala 1993}

Kujala UM, Jaakkola LH, Koskinen SK, Taimela S, Hurme M, Nelimarkka O. Scoring of patellofemoral disorders. Arthroscopy: The Journal of Arthroscopic and Related Surgery 1993;9(2):159-63. [MEDLINE: 8461073]

\section{Lefebvre 2011}

Lefebvre C, Manheimer E, Glanville J. Chapter 6: Searching for studies, Box 6.4.c. In: Higgins JPT, Green $S$ (editors). Cochrane Handbook for Systematic Reviews of Interventions Version 5.1.0 (updated March 2011). The Cochrane Collaboration, 2011. Available from www.cochrane-handbook.org.

\section{Lysholm 1982}

Lysholm J, Gillquist J. Evaluation of knee ligament surgery results with special emphasis on use of a scoring scale. 
American Journal of Sports Medicine 1982;10(3):150-4. [MEDLINE: 6896798]

\section{Mears 2001}

Mears SC, Cosgarea AJ. Surgical treatment options in patellofemoral disorders. Current Opinion in Orthopaedics 2001;12(2):167-73. [EMBASE: 2001135456]

\section{Merchant 2007}

Merchant ND, Bennett CH. Recent concepts in patellofemoral instability. Current Opinion in Orthopaedics 2007;18(2):153-60. [EMBASE: 2007090939]

\section{Nietosvaara 1994}

Nietosvaara Y, Aalto K, Kallio PE. Acute patellar dislocation in children: incidence and associated osteochondral fractures. Journal of Pediatric Orthopaedics 1994;14(4): 513-5. [MEDLINE: 8077438]

\section{Nikku 1997a}

Nikku R, Nietosvaara Y, Kallio PE, Aalto K, Michelsson JE. Operative versus closed treatment of primary dislocation of the patella. Similar 2-year results in 125 randomized patients. Acta Orthopaedica Scandinavica 1997;68(5): 419-23. [MEDLINE: 9385238]

Palmu 2008

Palmu S, Kallio PE, Donell ST, Helenius I, Nietosvaara Y. Acute patellar dislocation in children and adolescents: a randomised clinical trial. Journal of Bone \& Joint Surgery - American Volume 2008;90(3):463-70. [MEDLINE: 18310694]

Paxton 2003

Paxton EW, Fithian DC, Stone ML, Silva P. The reliability and validity of knee-specific and general health instruments in assessing acute patellar dislocation outcomes. American Journal of Sports Medicine 2003;31(4):487-92. [MEDLINE: 12860533]

Saccomanno 2012

Saccomanno M, Deriu L, Careri S, De Ieso C, Donati F, Milano G, et al. Surgical versus conservative treatment after acute patellar dislocation: A systematic review and meta-analysis. Abstracts of the 97th National Congress of the Italian Society of Orthopaedics and Traumatology; 2012 Nov 10-14; Rome, Italy. Journal of Orthopaedics and Traumatology, 2012.

\section{Scher 2010}

Scher DL, Owens BD, Sturdivant RX, Wolf JM. Incidence of joint hypermobility syndrome in a military population: impact of gender and race. Clinical Orthopaedics and Related Research 2010;458(7):1790-5.

Sillanpää 2012

Sillanpää PJ, Mäenpää HM. First-time patellar dislocation: surgery or conservative treatment?. Sports Medicine and Arthroscopy Reviews 2012;20(3):128-35. [DOI: 10.1097/ JSA.0b013e318256bbe5]

\section{Smith 2007}

Smith TO, Walker J, Russell N. Outcomes of medial patellofemoral ligament reconstruction for patellar instability: a systematic review. Knee Surgery, Sports

Traumatology, Arthroscopy 2007;15(11):1301-14.

\section{Smith 2008}

Smith TO, Leigh D. Outcomes following trochleoplasty for patellar instability with trochlear dysplasia: A systematic review. European Journal of Orthopaedic Surgery and Traumatology 2008;18(6):425-33. [EMBASE: 2008375916]

\section{Smith 2010}

Smith TO, Davies L, Chester R, Clark A, Donell ST. A systematic review of physiotherapy following lateral patellar dislocation. Physiotherapy 2010;96:269-81. [DOI: 10.1016/j.physio.2010.02.006]

\section{Smith 2011}

Smith TO, Song F, Donell ST, Hing CB. Operative versus non-operative management of patellar dislocation. A metaanalysis. Knee Surgery, Sports Traumatology, Arthroscopy 2011;19(6):988-98. [PUBMED: 21234544]

\section{Song 2010}

Song F, Parekh S, Hooper L, Loke YK, Ryder J, Sutton AJ, et al. Dissemination and publication of research findings: an updated review of related biases. Health Technology Assessment 2010;14(8):1-193. [DOI: 10.3310/hta14080]

\section{Stefancin 2007}

Stefancin JJ, Parker RD. First-time traumatic patellar dislocation. A systematic review. Clinical Orthopaedics and Related Research 2007;455:93-101. [MEDLINE: 17279039]

\section{Strugnell 2014}

Strugnell C, Dunstan DW, Magliano DJ, Zimmet PZ, Shaw JE, Daly RM. Influence of age and gender on fat mass, fatfree mass and skeletal muscle mass among Australian adults: The Australian Diabetes, Obesity and Lifestyle Study (AusDiab). Journal of Nutrition, Health and Ageing 2014;18 (5):540-6.

Tegner 1985

Tegner Y, Lysholm J. Rating systems in the evaluation of knee ligament injuries. Clinical Orthopaedics and Related Research 1985;(198):43-9. [MEDLINE: 4028566]

Tsai 2012

Tsai CH, Hsu CJ, Hung CH, Hsu HC. Primary traumatic patellar dislocation. Journal of Orthopaedic Surgery and Research 2012;6(7):21. [DOI: 10.1186/1749-799X-7-21]

Ware 1996

Ware J Jr, Kosinski M, Keller SD. A 12-Item Short-Form Health Survey: construction of scales and preliminary tests of reliability and validity. Medical Care 1996;34(3):220-33. [MEDLINE: 8628042]

\section{Woo 1998}

Woo R, Busch MT. Management of patellar instability in children. Operative Techniques in Sports Medicine 1998;6 (4):247-58.

References to other published versions of this review 
Hing 2011

Hing CB, Smith TO, Donell S, Song F. Surgical versus non-surgical interventions for treating patellar dislocation.

Cochrane Database of Systematic Reviews 2011, Issue 11.

[DOI: 10.1002/14651858.CD008106.pub2]

* Indicates the major publication for the study 


\section{CHARACTERISTICS OFSTUDIES}

\section{Characteristics of included studies [ordered by study ID]}

\section{Bitar 2012}

\begin{tabular}{ll}
\hline Methods & $\begin{array}{l}\text { Single-centre RCT } \\
\text { Randomisation method: drawing of paper slips } \\
\text { Follow-up: minimum was two years; mean follow-up was } 44 \text { months (range } 24 \text { to } 61 \\
\text { months) }\end{array}$ \\
\hline Participants & Trial performed in Brazil. Recruitment from 2003 to 2006 \\
& $\mathrm{~N}=42$ but presented in the text $\&$ table as 39 participants (41 knees) with 3 others "lost \\
in the follow-up period" & Inclusion criteria: acute (up to three weeks post-injury) primary patellar dislocation with \\
a history of laterally displaced patella and on physical examination: tenderness of the \\
medial retinaculum, a positive apprehension test, effusion or haemarthrosis of the knee \\
joint attributed to a patellar dislocation. Confirmation of diagnosis and assessment of \\
injury to the MPFL made using magnetic resonance imaging (MRI) \\
Exclusion criteria: participants excluded with previous history of knee surgery or serious \\
knee lesion including patellar dislocation or symptoms of patellar instability; coexistence \\
of tibiofemoral ligament injury requiring repair; large osteochondral fragments (diam- \\
eter $>15$ mm) requiring fixation; conditions associated with serious neuromuscular or \\
congenital disease; participants younger than 12 years of age; a non-traumatic patellar \\
dislocation (e.g. dislocation during gait or squatting with moderate stress on the knee); \\
inability/unwillingness to provide consent or comply with treatment protocol
\end{tabular}

patellar ligament from the tibial tuberosity to the adductor tubercle of the femoral condyle, attached to this point with an absorbable interference screw. Suture attachment of the rotated graft with the distal end of the vastus medialis muscle also performed. No lateral release or other procedure undertaken. Post-surgical rehabilitation: all surgical participants were immobilised for three weeks in a knee immobiliser (knee position not stated). During this period, isometric quadriceps strengthening exercises, analgesics, cryotherapy and electronic stimulation was permitted. Immediate weight-bearing permitted post-operatively, and passive knee range of motion exercises performed by a physiotherapist. At the third post-operative week, the knee immobiliser was dispelled and knee range of motion, proprioception and closed kinetic chain exercises commenced; these were progressed to open kinetic chain exercises over time. The overall objective was to progress surgical participants to return to previous sporting activities in approximately 10 to 12 weeks post-operation

Non-surgery $(\mathrm{N}=18$ participants/20 knees; mean age $24.1 ; 9$ females $(11$ knees $) / 9$ males)

Intervention: non-weight-bearing immobilised in a extension brace for three weeks, followed by a physiotherapy programme consisting of quadriceps strengthening and knee range of motion exercises. During the initial three weeks of immobilisation, participants were provided with analgesia, cryotherapy and electrical stimulation. Weight-bearing was permitted after the three weeks of immobilisation. Initially proprioceptive and closed kinetic chain exercises were prescribed. These were progressed to open kinetic chain 
Bitar 2012 (Continued)

exercises, with the overall objective to progress the participants to their previous sporting activities within 16 to 24 weeks post-commencement of non-operative rehabilitation

Outcomes $\quad$ Follow-up: mean 44 months (range 24 to 61 months)

Outcomes collected included: Kujala patellofemoral disorders score; recurrent patellar dislocation; episodes of patellar subluxation; and participant satisfaction

Notes

Power calculation used, requiring 22 in each group. Intention-to-treat analysis principles were adopted. No strategy was established to analyse or impute missing data

Personal communication with Dr A Bitar (25th October 2013) who reviewed the search results

\section{Risk of bias}

\begin{tabular}{|c|c|c|}
\hline Bias & Authors' judgement & Support for judgement \\
\hline $\begin{array}{l}\text { Random sequence generation (selection } \\
\text { bias) }\end{array}$ & Unclear risk & $\begin{array}{l}\text { Quote: "For randomisation...we con- } \\
\text { ducted a draw for the } 2 \text { groups" (page 115) } \\
\text {. No report of how sequence was generated }\end{array}$ \\
\hline Allocation concealment (selection bias) & Unclear risk & $\begin{array}{l}\text { No reference was made to concealment } \\
\text { of allocation during randomisation (page } \\
115 \text { ) }\end{array}$ \\
\hline $\begin{array}{l}\text { Blinding of participants and personnel } \\
\text { (performance bias) } \\
\text { All outcomes }\end{array}$ & High risk & $\begin{array}{l}\text { Blinding of participants and personnel not } \\
\text { reported, but extremely unlikely }\end{array}$ \\
\hline $\begin{array}{l}\text { Blinding of outcome assessment (detection } \\
\text { bias) } \\
\text { All outcomes }\end{array}$ & High risk & $\begin{array}{l}\text { Blinding of outcome assessors not reported, } \\
\text { and unlikely. Participants completing the } \\
\text { Kujala questionnaire and reporting recur- } \\
\text { rent dislocation/subluxation were clearly } \\
\text { unblinded }\end{array}$ \\
\hline Incomplete outcome data (attrition bias) & High risk & $\begin{array}{l}\text { Three lost to follow-up post-randomisation } \\
\text { and data not included. The follow-up was } \\
38 \text { months for the surgical management } \\
\text { group and } 48 \text { months for the non-surgical } \\
\text { management group, which is a likely source } \\
\text { of bias }\end{array}$ \\
\hline Selective reporting (reporting bias) & High risk & $\begin{array}{l}\text { No protocol available but the planned out- } \\
\text { comes defined in the Methods section (page } \\
115 \text { to } 116 \text { ) were reported in the Results } \\
\text { section (page } 117 \text { to } 118 \text { ). Adverse effects } \\
\text { of surgery were not reported }\end{array}$ \\
\hline
\end{tabular}


Randomisation method: blind drawing of slips of paper allocating group

Follow-up: "minimum follow-up time of 25 months" (listed as part of inclusion criteria) to maximum 60 months; mean 40.4 months in surgical group and 36.3 months in nonsurgery group

Location and person who randomised or assessed not stated

Participants

Interventions
Trial performed in Brazil. The period in which the study was undertaken was not stated $\mathrm{N}=33$ participants

Inclusion criteria: primary patellar dislocation with a convincing history of traumatic dislocation, requirement for reduction

Exclusion criteria: osteochondral fracture, patellar fracture, previous knee surgery

Surgery ( $\mathrm{N}=17$; mean age $24.6 ; 11$ females $/ 6$ males)

Intervention: arthroscopic MPFL repair. Post-operative rehabilitation: three weeks in a removable immobiliser and physiotherapy

Non-surgery ( $\mathrm{N}=16$; mean age 26.8; 9 females/7 males)

Intervention: immobilised in a cylinder cast for three weeks, followed by a physiotherapy programme consisting of strengthening exercises particularly of the vastus medialis obliquus. Hamstring and retinacular stretching begun after one month post-dislocation

Outcomes

Follow-up: aim between two and five years, mean 40.4 months in the surgery group and 36.3 months in the non-surgical group

Outcomes collected included: recurrent patellar dislocation, positive apprehension test, recurrent instability symptoms, Smillie test results, and the Kujala patellofemoral disorders score

Notes

Not concealed allocation; location and person who randomised not stated. No details provided on rehabilitation programme used. Sample size was not based on a power calculation. Number of surgeons not stated

Personal communication with Dr A Bitar who reviewed the updated search results (25th October 2013) and provided standard deviation values for Kujala patellofemoral disorders score results (19th January 2010)

\section{Risk of bias}

\section{Bias}

Random sequence generation (selection Unclear risk bias)

\section{Support for judgement}

Quote: "Patients were randomly divided into 2 groups by means of a drawing, by blindly selecting a slip of paper that assigned them to either the surgical treatment group or the conservative treatment group" (page 621)

No report of how sequence was generated

Allocation concealment (selection bias) Unclear risk
Quote "Patients were randomly divided into 2 groups by means of a drawing, by blindly selecting a slip of paper that as- 
Camanho 2009 (Continued)

signed them to either the surgical treatment group or the conservative treatment group" (page 621). Although blinding is mentioned, there is no mention of adequate safeguards

Blinding of participants and personnel High risk (performance bias)

Blinding of participants and personnel not reported, but extremely unlikely

All outcomes

Blinding of outcome assessment (detection High risk

bias)

All outcomes

Blinding of outcome assessors not reported, and unlikely. Participants completing questionnaires for the Kujala and Tegner scores were clearly unblinded

Incomplete outcome data (attrition bias) High risk

The title indicates that it is a study on the management of acute patellar dislocation, and the text (page 621) states "All were operated on less than 1 month after the trauma causing the lesion had occurred.” However, in the inclusion criteria we find "a minimum follow-up time of 25 months after the dislocation episode" (page 621), and in the exclusion criteria we find "followup after the first dislocation shorter than 24 months" (page 621). This appears to mean that randomised participants from both groups were excluded from the analysis, but there is no report of losses

Follow-up may have stretched from 25 to 60 months. Additionally, the follow-up was 40.4 months for the surgical management group and 36.3 months for the non-surgical management group; which may be a source of bias

Selective reporting (reporting bias) High risk

No protocol available but the planned outcomes defined in the methods section were reported. Adverse effects of surgery were not reported

Christiansen 2008

Methods

Single-centre RCT

Randomisation method: drawing of envelopes

Follow-up: two years

Surgical versus non-surgical interventions for treating patellar dislocation (Review)

Copyright $\odot 2015$ The Cochrane Collaboration. Published by John Wiley \& Sons, Ltd. 
Christiansen 2008 (Continued)

\begin{tabular}{ll}
\hline Participants & Trial performed in Denmark from April 1998 to September 2002 \\
$\mathrm{~N}=80$ participants (77 reported as 3 excluded as did not complete final follow-up) \\
Inclusion criteria: individuals with primary patellar dislocation, aged 13 to 30 years \\
Exclusion criteria: history of patellofemoral instability or pain; unable to follow treatment \\
regimen
\end{tabular}

Interventions

All participants underwent an arthroscopy

Surgery $(\mathrm{N}=42$; mean age $20.0 ; 18$ females $/ 24$ males $)$

Intervention: repair of the MPFL performed on average 50 days post-dislocation Postoperative rehabilitation: no information provided

Non-surgery ( $\mathrm{N}=35$; mean age $19.9 ; 17$ females/18 males)

Intervention: brace from zero to two weeks immobilised zero to 20 knee range of motion degrees

Outcomes

Follow-up: 2 years (also 2 and 6 weeks, and 1 year)

Outcomes collected included: incidence of re-dislocation at two years, Kujala patellofemoral disorders score, and the Knee Injury and Osteoarthritis Outcome Score (KOOS)

Notes

Power calculation used. Requiring 39 in each group. Intention-to-treat analysis principles were not adopted. Personal communication with Dr Martin Lind who reviewed the updated search results (22nd October 2013)

Risk of bias

\begin{tabular}{|c|c|c|}
\hline Bias & Authors' judgement & Support for judgement \\
\hline $\begin{array}{l}\text { Random sequence generation (selection } \\
\text { bias) }\end{array}$ & Unclear risk & $\begin{array}{l}\text { Quote: "Randomization between surgery } \\
\text { and conservative treatment was performed } \\
\text { by random drawing of } 100 \text { envelopes" } \\
\text { (page } 883 \text { ). No report of how sequence was } \\
\text { generated }\end{array}$ \\
\hline
\end{tabular}

Allocation concealment (selection bias) Unclear risk

Sealed envelope system. Quote: "Randomization between surgery and conservative treatment was performed by random drawing of 100 envelopes" (page 883), but no report of whether these were securely sealed and allocated sequentially

Blinding of participants and personnel High risk (performance bias)

All outcomes

Blinding is not mentioned in the study report. Treatment staff and participants unlikely to be blinded, as randomisation was conducted at arthroscopy. Aftercare clearly not identical in both groups (Quote: "Patients randomised to conservative treatment received no further treatment or brace usage” (page 882) 
Christiansen 2008 (Continued)

\begin{tabular}{l|l|l}
$\begin{array}{l}\text { Blinding of outcome assessment (detection } \\
\text { bias) }\end{array}$ & High risk & $\begin{array}{l}\text { Blinding of outcome assessors not reported, } \\
\text { and unlikely. Participants completing ques- }\end{array}$ \\
All outcomes & tionnaires for the Kujala and KOOS scores \\
& were clearly unblinded
\end{tabular}

Incomplete outcome data (attrition bias) Unclear risk

Three lost to follow-up post-randomisation (Figure 2) and data not included

Selective reporting (reporting bias) High risk

No protocol available but the planned outcomes defined in the methods section were reported, but did not include adverse effects of surgery

Nikku 1997

\begin{tabular}{|c|c|}
\hline Methods & $\begin{array}{l}\text { Multi-centre RCT } \\
\text { Quasi-randomisation using year of birth } \\
\text { Follow-up: mean } 25 \text { months (range } 20 \text { to } 45 \text { months); mean } 7 \text { years (range } 5.7 \text { to } 9.1 \\
\text { years), mean } 14 \text { years (range } 11 \text { to } 15 \text { years) for children only subgroup }\end{array}$ \\
\hline Participants & $\begin{array}{l}\text { Trial performed in Finland. Recruitment from January } 1991 \text { to December } 1992 \\
\mathrm{~N}=125 \text { participants (127 knees) } \\
\text { Inclusion criteria: primary lateral patellar dislocation where injury was less than } 14 \text { days } \\
\text { Exclusion criteria: previous major knee injury, previous knee surgery, ligament injuries } \\
\text { needing repair, osteochondral fractures needing fixation }\end{array}$ \\
\hline Interventions & $\begin{array}{l}\text { Surgery (N = 70; mean age } 19.5, \text { SD 9; } 52 \text { females } / 18 \text { males) } \\
\text { Intervention: medial reefing (18), repair or medial retinaculum (39) or augmentation of } \\
\text { MPFL (6) or lateral release (54) } \\
\text { Post-operative rehabilitation: thigh muscle exercises and full weight-bearing. If patellar } \\
\text { dislocatable on examination under anaesthesia, immobilised on splint/cast for three } \\
\text { weeks. Mobilisation started with orthosis for three weeks and used during sporting } \\
\text { activities for the first six months post-dislocation } \\
\text { Non-surgery ( } \mathrm{N}=55 \text {; mean age } 19.1, \mathrm{SD} 7.5 ; 30 \text { females } / 25 \text { males) } \\
\text { Intervention: identical rehabilitation programme to surgical group }\end{array}$ \\
\hline Outcomes & $\begin{array}{l}\text { Follow-up ( } 3 \text { time periods): mean } 25 \text { months (range } 20 \text { to } 45 \text { months); mean } 7 \text { years } \\
\text { (range } 5.7 \text { to } 9.1 \text { years); and, for a children-only subgroup, mean } 14 \text { years ( } 11 \text { to } 15 \\
\text { years) } \\
\text { Outcomes collected included: patient satisfaction with outcome, Lysholm knee score, } \\
\text { Hughston VAS knee score, Tegner activity score, recurrent dislocation rates, recurrent } \\
\text { subluxation rates; subsequent surgical intervention, performance tests consisting of timed } \\
\text { figure of eight running, one leg hop distance, maximum number of squat downs in } \\
\text { one minute, and subsequent pain on VAS, thigh circumference knee range of motion, } \\
\text { patellofemoral crepitus, apprehension test, prepatellar sensibility and scar sensibility }\end{array}$ \\
\hline
\end{tabular}

Surgical versus non-surgical interventions for treating patellar dislocation (Review) 
Nikku 1997 (Continued)

$\begin{array}{ll}\text { Notes } & \begin{array}{l}\text { Two orthopaedic consultants and two registrars did } 88 \% \text { of operations. Assessment } \\ \text { clinically performed by two surgeons. Intention-to-treat analysis principles were not } \\ \text { adopted. Sample size was not based on a power calculation. Confirmation gained from }\end{array} \\ \text { Prof Simon Donell that Palmu } 2008 \text { (which was previously included as a separate study) } \\ \text { reported the 14-year follow-up of a children-only (including adolescents) subgroup of } \\ \text { this trial (25th October 2014) (Donell 2014) }\end{array}$

Risk of bias

\begin{tabular}{l|l|l}
\hline Bias & Authors' judgement & Support for judgement \\
\hline $\begin{array}{l}\text { Random sequence generation (selection } \\
\text { bias) }\end{array}$ & High risk & $\begin{array}{l}\text { Quote: "randomization was based on the } \\
\text { year of birth (even/odd)" (page 420) }\end{array}$ \\
\hline $\begin{array}{l}\text { Allocation concealment (selection bias) } \\
\text { Blinding of participants and personnel } \\
\text { (performance bias) } \\
\text { All outcomes }\end{array}$ & High risk & $\begin{array}{l}\text { Quote: "randomization was based on the } \\
\text { year of birth (even/odd)" (page 420) }\end{array}$ \\
\hline & High & $\begin{array}{l}\text { Blinding is not mentioned in the study re- } \\
\text { port. Treatment staff and participants un- } \\
\text { likely to be blinded. To note though that: } \\
\text { Quote: "After-care was identical in both } \\
\text { groups" (page 420) }\end{array}$ \\
\hline
\end{tabular}

Blinding of outcome assessment (detection High risk bias)

All outcomes
Assessor/data collection blinding is not mentioned in the study report. Quote: "Recurrences were asked about twice: by a mailed questionnaire and by the examiner at the final evaluation” (page 420). Quote: "The clinical examination was performed by two of the authors (YN, RN)" (page 420)

Participants completing questionnaires for the Lysholm, Hughston VAS, Kujala and Tegner scores were clearly unblinded
Incomplete outcome data (attrition bias) Unclear risk

Quote: “123/125 patients attended the performance test and clinical examination. 2 patients returned only the questionnaires" (page 420-1)

However, there was mention of exclusions: " 4 had erroneous randomization and 1 was lost to follow-up"

Selective reporting (reporting bias) Unclear risk
No protocol available but the planned outcomes defined in the methods section were reported. Adverse effects of surgery were reported. The reporting of the children-only subgroup at 14 years (Palmu 2008) did not 
Nikku 1997 (Continued)

appear to have been pre-determined

Petri 2013

Methods

Multi-centre RCT

Randomisation method: sealed envelope system performed in the individual study centres

Follow-up: 24 months (questionnaire)

Participants

Trial performed in Germany

$\mathrm{N}=24$ participants

Inclusion criteria: isolated, unilateral first-time traumatic patellar dislocation; aged between 15 and 40 years of age; provided informed consent to participate

Exclusion criteria: recurrent dislocation; significant anatomical deformities (not specified); open injury; participants who were pregnant or lactating; an osteochondral fracture which required fixation

Interventions

Surgery ( $\mathrm{N}=12$; mean age $27.2 ; 4$ females/8 males)

Intervention: diagnostic arthroscopy performed, followed by open soft tissue repairs including mainly suture and optional tightening of ruptured medial structures. "MPFLplastics" were not performed. Lateral release was optional. Tibial tuberosity and bony correction was optional. Post-operative rehabilitation: a DonJoy range of motion brace was applied with 0 to 60 degrees extension-flexion permitted from weeks zero to three, increased to zero to 90 degrees extension-flexion permitted from weeks three to six. Participants were required to partial weight-bear for initial 3 weeks up to $15 \mathrm{~kg}$ on crutches, followed by progressions to full weight-bearing from week three onwards. No further information on rehabilitation provided

Non-surgery ( $\mathrm{N}=8$; mean age 21.6; 3 females/5 males)

Intervention: participants were provided with a DonJoy range of motion brace with zero to 60 degrees extension-flexion permitted from weeks zero to three, increased to zero to 90 degrees extension-flexion permitted from weeks three to six post-randomisation. Participants were required to partial weight-bear for initial three weeks up to $15 \mathrm{~kg}$ on crutches, followed by progressions to full weight-bearing from week three onwards. No further information on rehabilitation provided

Outcomes

Follow-up: 2 years (also 6 and 12 months).

Outcomes recorded included: Kujala patellofemoral disorders score; recurrent dislocation; episodes of patellar subluxation; and participant satisfaction

Notes

Sample size was not based on a power calculation. No statement on intention-to-treat analysis. No attempt was made to analyse missing data using imputation techniques. Personal communication with Dr P Balcarek (27th October 2013) and Dr M Petri who reviewed the updated search results (25th October 2013)

Risk of bias

Bias

Authors' judgement

Support for judgement

Surgical versus non-surgical interventions for treating patellar dislocation (Review)

Copyright $\odot 2015$ The Cochrane Collaboration. Published by John Wiley \& Sons, Ltd. 
Petri 2013 (Continued)

\begin{tabular}{|c|c|c|}
\hline $\begin{array}{l}\text { Random sequence generation (selection } \\
\text { bias) }\end{array}$ & Low risk & $\begin{array}{l}\text { Quote: "With use of a sealed envelope } \\
\text { method utilising a software generated block } \\
\text { randomisation patients were randomised in } \\
\text { the individual centres" (page 210) }\end{array}$ \\
\hline Allocation concealment (selection bias) & Low risk & $\begin{array}{l}\text { Quote: "With use of a sealed envelope } \\
\text { method utilising a software generated block } \\
\text { randomisation patients were randomised in } \\
\text { the individual centres" (page 210) }\end{array}$ \\
\hline $\begin{array}{l}\text { Blinding of participants and personnel } \\
\text { (performance bias) } \\
\text { All outcomes }\end{array}$ & High risk & $\begin{array}{l}\text { Participant blinding is not mentioned in } \\
\text { the study report. Due to the nature of the } \\
\text { interventions, treatment staff and partici- } \\
\text { pants unlikely to be blinded }\end{array}$ \\
\hline $\begin{array}{l}\text { Blinding of outcome assessment (detection } \\
\text { bias) } \\
\text { All outcomes }\end{array}$ & High risk & $\begin{array}{l}\text { All outcomes were self-reported (thought } \\
\text { questionnaires) by the participants. Blind- } \\
\text { ing of participants not reported, but clearly } \\
\text { unblinded }\end{array}$ \\
\hline Incomplete outcome data (attrition bias) & Unclear risk & $\begin{array}{l}\text { Two participants lost to follow-up in the } \\
\text { surgical group; two participants in the non- } \\
\text { surgical group } \\
\text { Three participants had moved out of the } \\
\text { area, whilst contact data for one participant } \\
\text { were incomplete }\end{array}$ \\
\hline Selective reporting (reporting bias) & High risk & $\begin{array}{l}\text { No protocol available but the planned out- } \\
\text { comes defined in the methods section (page } \\
210 \text { ) were presented in the Results section } \\
\text { (pages } 211-2 \text { ). Adverse effects of surgery } \\
\text { were not reported }\end{array}$ \\
\hline
\end{tabular}

Sillanpaa 2009

Methods

Single-centre RCT

Randomisation method: sealed envelopes

Follow-up: median 7 years ( 6 to 9 years)

Participants

Trial performed in Finland. Recruitment from 1998 to 2000

$\mathrm{N}=40$ participants (all military recruits)

Inclusion criteria: individuals with a primary acute traumatic patellar dislocation

Exclusion criteria: previous subluxation, pre-existing ipsilateral or contralateral knee pathology, previous ligament injury or fracture of the involved knee, or large osteochondral lesion requiring open surgery

Surgical versus non-surgical interventions for treating patellar dislocation (Review) 


$\begin{array}{ll}\text { Interventions } & \text { Surgery }(\mathrm{N}=18 ; \text { mean age } 20.0 ; 1 \text { female/17 males) } \\ \text { Intervention: medial reefing and repair of MPFL (14); Roux-Goldthwaite procedure } \\ (4) \text { arthroscopic repair of osteochondral fracture (6). Post-operative rehabilitation: no } \\ \text { information provided } \\ \text { Non-surgery }(\mathrm{N}=22 \text {; mean age } 20.0 ; 2 \text { females/20 males) } \\ \text { Intervention: knee orthosis, guided isometric quadriceps exercises. First three weeks } \\ \text { immobilised zero to } 30 \text { degrees knee flexion, three to six weeks immobilised form zero } \\ \text { to } 90 \text { degrees and free range of motion from six weeks onwards. (All participants of this } \\ \text { group received knee aspiration to relieve pain and four underwent arthroscopic removal } \\ \text { of an osteochrondral fragment) }\end{array}$

\begin{tabular}{|c|c|c|}
\hline Bias & Authors' judgement & Support for judgement \\
\hline $\begin{array}{l}\text { Random sequence generation (selection } \\
\text { bias) }\end{array}$ & Unclear risk & $\begin{array}{l}\text { Quote: "...military recruits who had been } \\
\text { admitted to a military hospital because of } \\
\text { an acute primary traumatic patellar disloca- } \\
\text { tion were randomized to treatment" (page } \\
\text { 264). No report of how sequence was gen- } \\
\text { erated }\end{array}$ \\
\hline Allocation concealment (selection bias) & Unclear risk & $\begin{array}{l}\text { Quote: "Written informed consent was } \\
\text { obtained from each patient. With use of } \\
\text { a sealed-envelope method, forty patients } \\
\text { were randomly allocated to two treat- } \\
\text { ment groups: (1) initial patellar stabiliza- } \\
\text { tion surgery and ( } 2 \text { ) non-operative treat- } \\
\text { ment with a knee orthosis (as well as arthro- } \\
\text { scopic removal of an osteochondral frag- } \\
\text { ment if necessary)" (page 264) No mention } \\
\text { of adequate safeguards }\end{array}$ \\
\hline
\end{tabular}


Blinding of participants and personnel High risk (performance bias)

All outcomes
Blinding of personnel or participants was not described. Quote: “The post-injury or postoperative rehabilitation protocols were identical for the two groups" (page 264) . However, Quote: "Four patients in the nonoperatively treated group underwent arthroscopic removal of an osteochondral fragment, but no additional procedures were performed. Since primary traumatic patellar dislocations are frequently associated with osteochondral fractures, we believe that performing arthroscopy initially in some patients may be unavoidable, even in a randomized study. Ten patients (four treated nonoperatively and six treated with surgical stabilization) had removable fragments, and the osteochondral fractures were treated identically (i.e. with arthroscopic removal of the fragments) in the two treatment groups" (page 266)

Blinding of outcomes assessment not described. Participants completing questionnaires for the Kujala and Tegner scores were clearly unblinded

One participant lost from each group: one participant missing had moved to another country, and one could not be reached for follow-up assessment

No protocol available but the planned outcomes defined in the methods section were reported. Adverse effects of surgery were not reported

MPFL $=$ medial patellofemoral ligament

RCT $=$ randomised controlled trial

VAS $=$ visual analogue scale 
Characteristics of excluded studies [ordered by study ID]

\begin{tabular}{ll}
\hline Study & Reason for exclusion \\
\hline Apostolovic 2011 & Not a randomised controlled trial \\
\hline Arnbjörnsson 1992 & Not a randomised controlled trial \\
\hline Buchner 2005 & Not a randomised controlled trial \\
\hline Cash 1988 & Not a randomised controlled trial \\
\hline Marcacci 1995 & Not a randomised controlled trial \\
\hline Savarese 1990 & Not a randomised controlled trial \\
\hline Sillanpää 2008a & Not a randomised controlled trial. All received some operative procedure \\
\hline Sillanpää 2008b & Not a randomised controlled trial \\
\hline
\end{tabular}

\section{Characteristics of ongoing studies [ordered by study ID]}

\section{ISRCTN39959729}

Trial name or title Conservative versus arthroscopic refixation of the medial patellofemoral ligament (MPFL) after traumatic first time dislocation of the patella in children: a prospective randomised study

\begin{tabular}{ll}
\hline Methods & $\begin{array}{l}\text { Single-centre RCT } \\
\text { Participants randomised to either non-operative (orthosis) or operative treatment. Randomisation was made } \\
\text { directly after the diagnostic arthroscopy with the participant still under general anaesthetic }\end{array}$ \\
\hline Participants & $\begin{array}{l}\text { Trial performed in Sweden } \\
\mathrm{N}=64 \\
\text { Inclusion criteria: } \\
\text { 1. Children } 9 \text { to } 14 \text { years of age } \\
\text { 2. Admitted to the emergency room (ER) with haemarthrosis after a traumatic first time patellar dislocation } \\
\text { 3. The diagnosis is based on clinical examination, magnetic resonance imaging (MRI) and arthroscopy } \\
\text { 4. The arthroscopy is the final confirmation of the diagnosis, and it gives a detailed description of the MPFL } \\
\text { injury and possible osteochrondral lesions } \\
\text { 5. Prior to the arthroscopy, the patients are asked to participate in the study } \\
\text { 6. The patients who have given informed consent } \\
\text { Exclusion criteria: } \\
\text { 1. Previous significant injury to the same knee including patellar dislocation, systemic joint disease or syn- } \\
\text { dromes affecting the knee joint } \\
\text { 2. Osteochondral lesion }>1 \mathrm{~cm} \text { on weight-bearing area that needs open reduction and fixation }\end{array}$
\end{tabular}




\section{ISRCTN39959729 (Continued)}

\begin{tabular}{l} 
Interventions \\
$\begin{array}{l}\text { Surgical group: Post-randomisation, the operation continued with an arthroscopic repair of the MPFL, with } \\
\text { refixation with anchors. Paticipants were then placed with the knee in full extension in a plaster cast for four } \\
\text { weeks. Following this, physiotherapy prescribed until participants have regained knee function. No further } \\
\text { information on rehabilitation was provided } \\
\text { Non-surgical group: Post-randomisation, the operation finished and the participant was placed in an orthosis } \\
\text { for four weeks. Following this, participants were referred to physiotherapy where they received treatment until } \\
\text { they had regained knee function. No further information on rehabilitation was provided }\end{array}$ \\
\hline
\end{tabular}

Outcomes

Follow-up period was two years post-randomisation. Outcomes collected include: recurrent patellar dislocation; post-randomisation complications; knee-examination (clinical and radiological evaluating Q-angle/TTTG distance, patella alta, patellar tilt, trochlea dysplasia, and joint mobility according to the Beighton score) ; joint range of motion; participant-administrated scores to evaluate activity, subjective knee function (i.e. Lysholm Knee Score, Tegner Activity Scale, Knee Osteoarthritis Outcome score (KOOS)-Child and Kujala patellofemoral disorders score; quality of life for children (i.e. EQ-5D-Y); objective knee function assessing hop-tests and "knee bending/30s-test"; and visual analogue scales (VAS) activity-related pain

Starting date

09/12/2009

Contact information Dr Per-Mats Janarv; Department of Pediatric Orthopedics, Astrid Lindgren Children's Hospital, Karolinska University Hospital, Stockholm, 17176, Sweden

Notes

The study is currently in the final data collection phase and will be closed after a two-year follow-up, which was expected to be in April 2014 (personal communication, Dr Per-Mats)

Current Controlled Trials Page: Janarv Trial 2009-2014

RCT: randomised controlled trial 
DATA AND ANALYSES

\section{Comparison 1. Surgical versus non-surgical management}

\begin{tabular}{|c|c|c|c|c|}
\hline Outcome or subgroup title & $\begin{array}{l}\text { No. of } \\
\text { studies }\end{array}$ & $\begin{array}{c}\text { No. of } \\
\text { participants }\end{array}$ & Statistical method & Effect size \\
\hline $\begin{array}{l}1 \text { Number of participants } \\
\text { sustaining recurrent patellar } \\
\text { dislocation }\end{array}$ & 6 & & Risk Ratio (M-H, Fixed, 95\% CI) & Subtotals only \\
\hline 1.1 Two to five years follow-up & 5 & 294 & Risk Ratio (M-H, Fixed, 95\% CI) & $0.53[0.33,0.87]$ \\
\hline 1.2 Six to nine years follow-up & 2 & 165 & Risk Ratio (M-H, Fixed, 95\% CI) & $0.67[0.42,1.08]$ \\
\hline 1.314 years follow-up & 1 & 64 & Risk Ratio (M-H, Fixed, 95\% CI) & $0.93[0.67,1.30]$ \\
\hline $\begin{array}{l}2 \text { Tegner activity score ( } 0 \text { to } 10 \text { : } \\
\text { best score) }\end{array}$ & 2 & & Mean Difference (IV, Fixed, 95\% CI) & Totals not selected \\
\hline $\begin{array}{l}2.1 \text { Two years ( } 20 \text { to } 45 \\
\text { months) follow-up }\end{array}$ & 1 & & Mean Difference (IV, Fixed, 95\% CI) & $0.0[0.0,0.0]$ \\
\hline 2.2 Six to nine years follow-up & 1 & & Mean Difference (IV, Fixed, 95\% CI) & $0.0[0.0,0.0]$ \\
\hline 2.314 years follow-up & 1 & & Mean Difference (IV, Fixed, 95\% CI) & $0.0[0.0,0.0]$ \\
\hline $\begin{array}{l}3 \text { KOOS (0 to } 100 \text { : best outcome) } \\
\text { at two years follow-up }\end{array}$ & 1 & & Mean Difference (IV, Fixed, 95\% CI) & Totals not selected \\
\hline 3.1 Symptoms & 1 & & Mean Difference (IV, Fixed, 95\% CI) & $0.0[0.0,0.0]$ \\
\hline 3.2 Pain & 1 & & Mean Difference (IV, Fixed, 95\% CI) & $0.0[0.0,0.0]$ \\
\hline 3.3 Activities of Daily Living & 1 & & Mean Difference (IV, Fixed, 95\% CI) & $0.0[0.0,0.0]$ \\
\hline 3.4 Sports and recreation & 1 & & Mean Difference (IV, Fixed, 95\% CI) & $0.0[0.0,0.0]$ \\
\hline 3.5 Quality of life & 1 & & Mean Difference (IV, Fixed, 95\% CI) & $0.0[0.0,0.0]$ \\
\hline $\begin{array}{l}4 \text { Lysholm score ( } 0 \text { to } 100 \text { : best } \\
\text { score) at two years ( } 20 \text { to } 45 \\
\text { months) follow-up }\end{array}$ & 1 & & Mean Difference (IV, Fixed, 95\% CI) & Totals not selected \\
\hline $\begin{array}{l}5 \text { Hughston VAS patellofemoral } \\
\text { score ( } 28 \text { to } 100 \text { : best outcome) }\end{array}$ & 1 & & Mean Difference (IV, Fixed, 95\% CI) & Totals not selected \\
\hline $\begin{array}{l}5.1 \text { Two years ( } 20 \text { to } 45 \\
\text { months) follow-up }\end{array}$ & 1 & & Mean Difference (IV, Fixed, 95\% CI) & $0.0[0.0,0.0]$ \\
\hline 5.214 years follow-up & 1 & & Mean Difference (IV, Fixed, 95\% CI) & $0.0[0.0,0.0]$ \\
\hline $\begin{array}{l}6 \text { Kujala patellofemoral disorders } \\
\text { score ( } 0 \text { to } 100 \text { : best outcome) }\end{array}$ & 6 & & Mean Difference (IV, Random, 95\% CI) & Subtotals only \\
\hline 6.1 Two to five years follow-up & 4 & 171 & Mean Difference (IV, Random, 95\% CI) & $13.93[5.33,22.53]$ \\
\hline 6.2 Six to nine years follow-up & 2 & 167 & Mean Difference (IV, Random, 95\% CI) & $-3.25[-10.61,4.11]$ \\
\hline 6.314 years follow-up & 1 & 64 & Mean Difference (IV, Random, 95\% CI) & $-1.0[-8.60,6.60]$ \\
\hline $\begin{array}{l}7 \text { Kujala patellofemoral disorders } \\
\text { score (0 to } 100 \text { : best outcome): } \\
\text { subgroup analysis }\end{array}$ & 1 & 41 & Mean Difference (IV, Fixed, 95\% CI) & $16.73[7.43,26.03]$ \\
\hline 7.1 Male & 1 & 21 & Mean Difference (IV, Fixed, 95\% CI) & $15.5[3.64,27.36]$ \\
\hline 7.2 Female & 1 & 20 & Mean Difference (IV, Fixed, 95\% CI) & $18.70[3.71,33.69]$ \\
\hline $\begin{array}{l}8 \text { Return to former activities: work } \\
\text { and sports }\end{array}$ & 1 & & Risk Ratio (M-H, Fixed, 95\% CI) & Totals not selected \\
\hline $\begin{array}{l}9 \text { Knee pain (VAS } 0 \text { to } 10 \text { : worst } \\
\text { outcome) }\end{array}$ & 2 & & Mean Difference (IV, Fixed, 95\% CI) & Totals not selected \\
\hline $\begin{array}{l}9.1 \text { Two years ( } 20 \text { to } 45 \\
\text { months) follow-up }\end{array}$ & 1 & & Mean Difference (IV, Fixed, 95\% CI) & $0.0[0.0,0.0]$ \\
\hline
\end{tabular}

Surgical versus non-surgical interventions for treating patellar dislocation (Review)

Copyright @ 2015 The Cochrane Collaboration. Published by John Wiley \& Sons, Ltd. 
9.2 Six to nine years follow-up $\quad 1$

10 Patient satisfaction (reported 2 good or excellent)

10.1 Two years (20 to 45

months) follow-up

10.2 Six to nine years

follow-up

10.314 years follow-up

11 Number of participants

sustaining recurrent patellar

subluxation

11.1 Two to five years

follow-up

11.2 Six to nine years

follow-up

12 Number of participants sustaining any episode of instability

12.1 Two to five years follow-up

12.2 Six to nine years follow-up

13 Number of participants who underwent subsequent surgery

13.1 Two years (20 to 45 months) follow-up

13.2 Six to nine years follow-up

13.314 years follow-up

5

4

4

3
Mean Difference (IV, Fixed, 95\% CI)

Risk Ratio (M-H, Fixed, 95\% CI)

144 Risk Ratio (M-H, Fixed, 95\% CI)

127 Risk Ratio (M-H, Fixed, 95\% CI)

60 Risk Ratio (M-H, Fixed, 95\% CI)

Risk Ratio (M-H, Fixed, 95\% CI)

216 Risk Ratio (M-H, Fixed, 95\% CI)

165 Risk Ratio (M-H, Fixed, 95\% CI)

Risk Ratio (M-H, Fixed, 95\% CI)

197 Risk Ratio (M-H, Fixed, 95\% CI)

165 Risk Ratio (M-H, Fixed, 95\% CI)

Risk Ratio (M-H, Fixed, 95\% CI)

125

Risk Ratio (M-H, Fixed, 95\% CI)

165

Risk Ratio (M-H, Fixed, 95\% CI)

64
$0.0[0.0,0.0]$

Subtotals only

$1.03[0.83,1.29]$

$0.83[0.68,1.02]$

$0.88[0.63,1.22]$

Subtotals only

$0.61[0.35,1.07]$

$0.98[0.60,1.58]$

Subtotals only

$0.44[0.27,0.72]$

$0.80[0.62,1.03]$

Subtotals only

$1.05[0.48,2.31]$

$1.06[0.59,1.89]$

$1.13[0.63,2.04]$ 


\section{Analysis I.I. Comparison I Surgical versus non-surgical management, Outcome I Number of participants}

sustaining recurrent patellar dislocation.

Review: Surgical versus non-surgical interventions for treating patellar dislocation

Comparison: I Surgical versus non-surgical management

Outcome: I Number of participants sustaining recurrent patellar dislocation

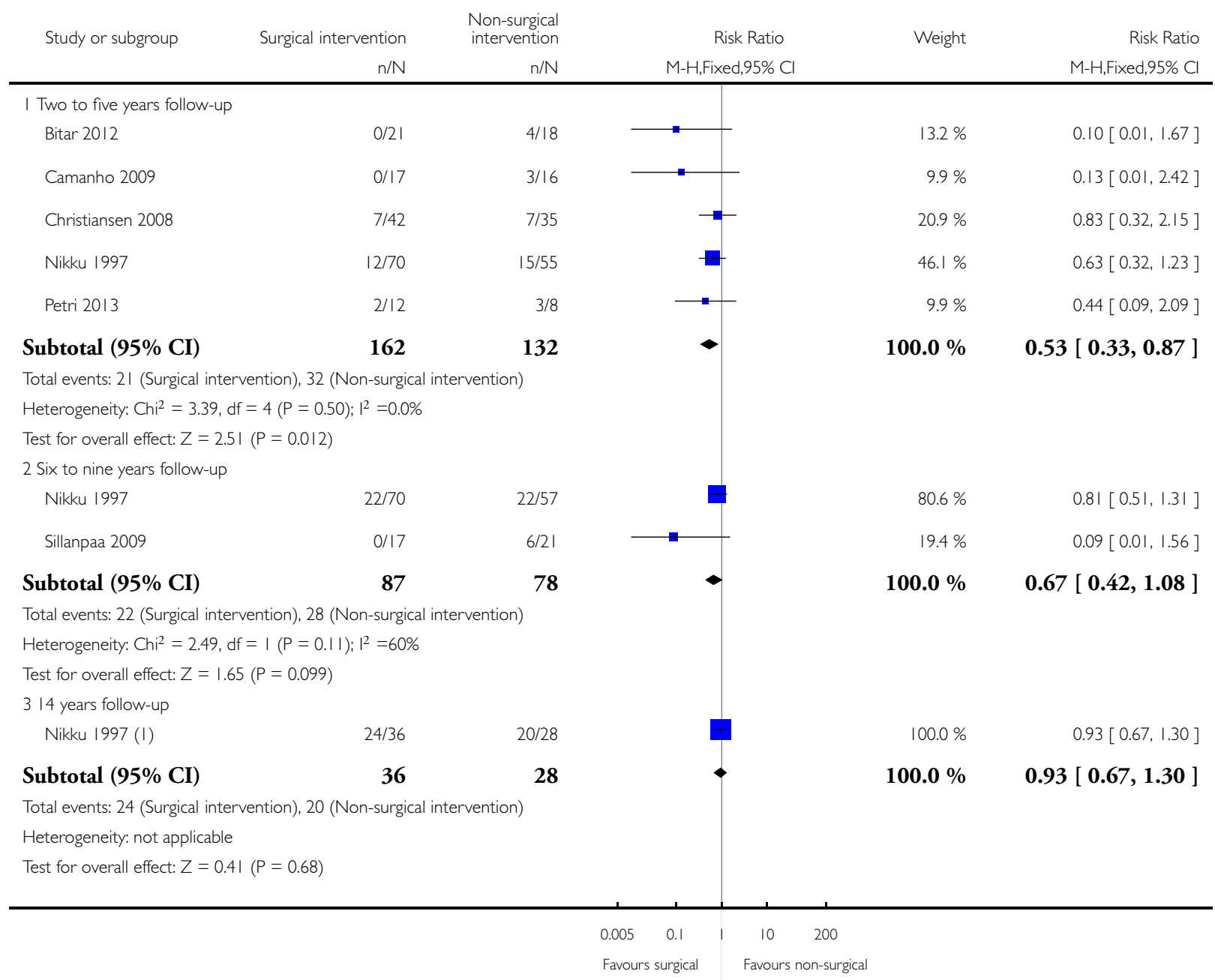

(I) Children only subgroup 
Analysis I.2. Comparison I Surgical versus non-surgical management, Outcome 2 Tegner activity score (0 to 10: best score).

Review: Surgical versus non-surgical interventions for treating patellar dislocation

Comparison: I Surgical versus non-surgical management

Outcome: 2 Tegner activity score (0 to I0: best score)

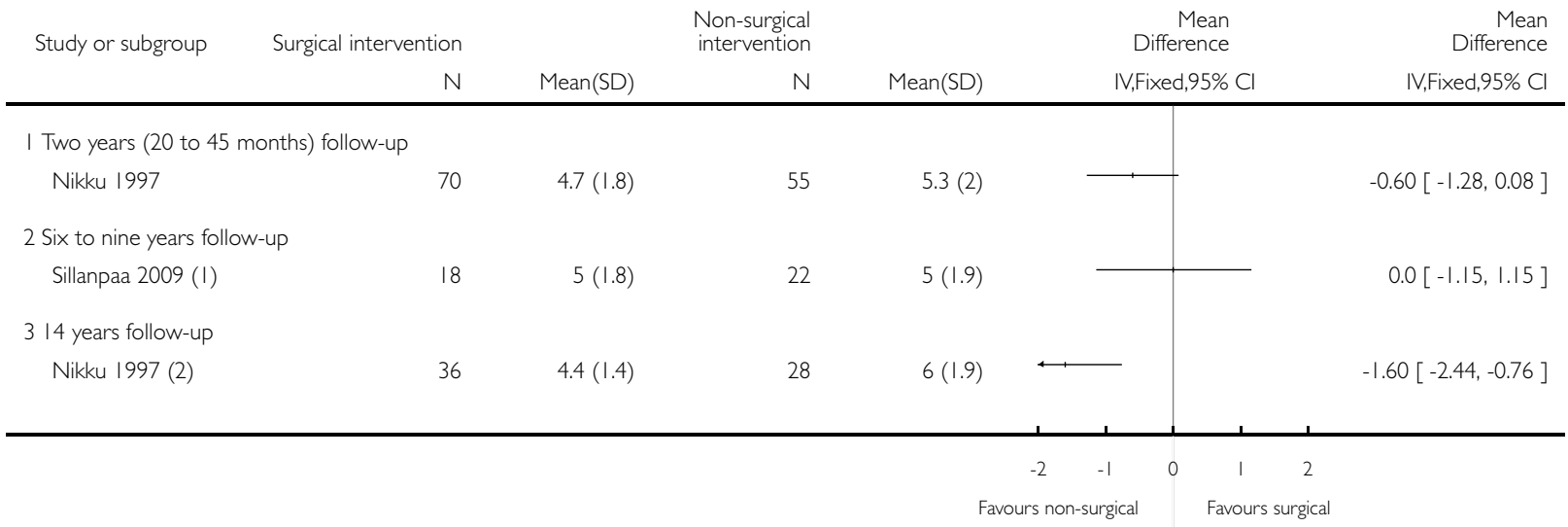

(I) Standard deviation values obtained from authors in 2010 (Sillanpaa P, personal communication).

(2) Children only subgroup 
Analysis I.3. Comparison I Surgical versus non-surgical management, Outcome 3 KOOS (0 to I00: best outcome) at two years follow-up.

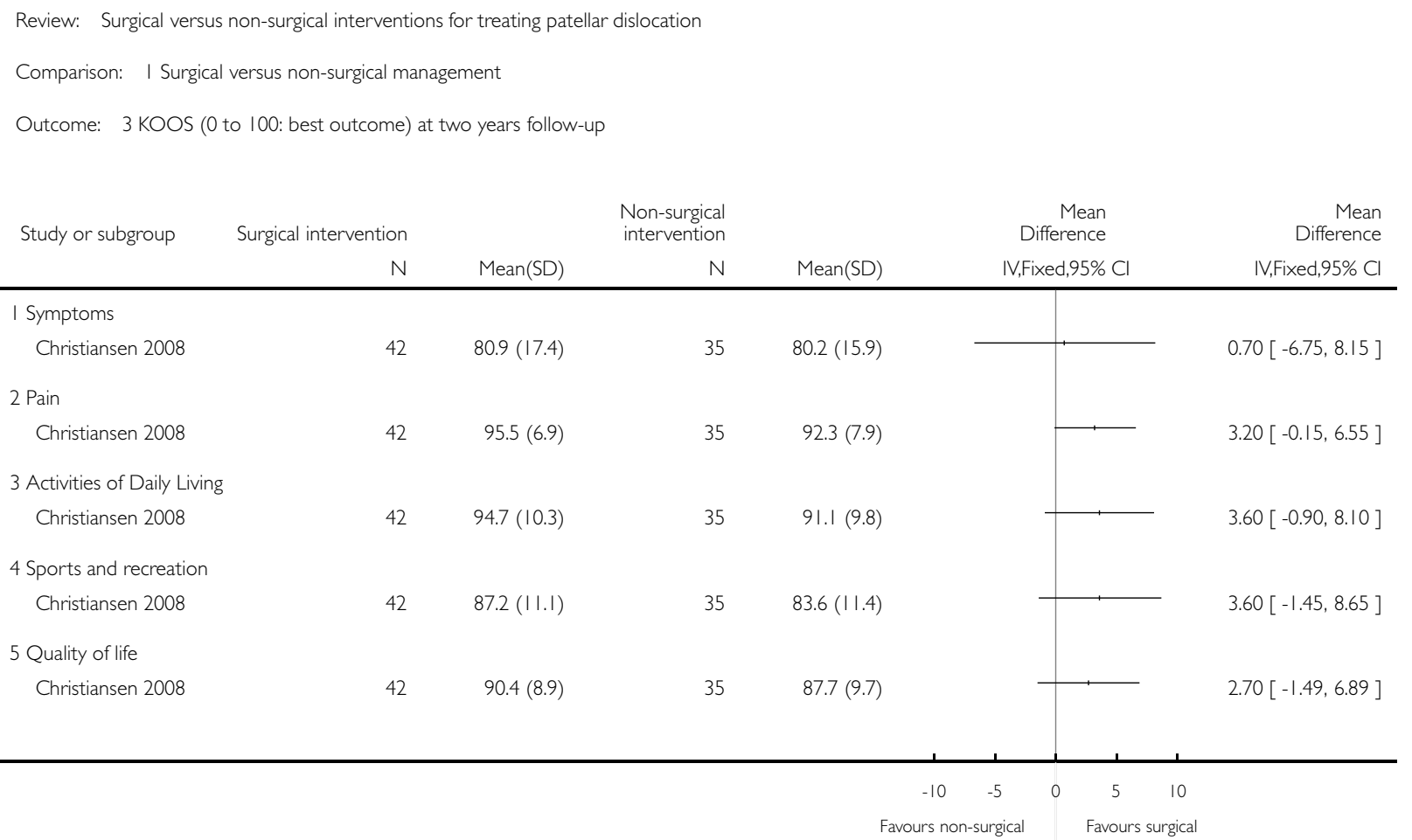

Analysis I.4. Comparison I Surgical versus non-surgical management, Outcome 4 Lysholm score (0 to I00: best score) at two years ( 20 to 45 months) follow-up.

Review: Surgical versus non-surgical interventions for treating patellar dislocation

Comparison: I Surgical versus non-surgical management

Outcome: 4 Lysholm score (0 to 100: best score) at two years (20 to 45 months) follow-up

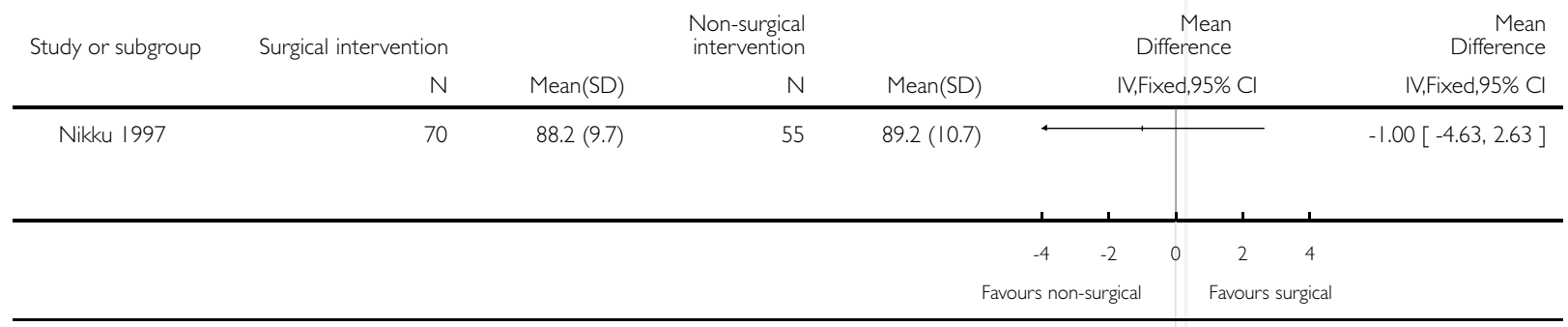

Surgical versus non-surgical interventions for treating patellar dislocation (Review) 


\section{Analysis I.5. Comparison I Surgical versus non-surgical management, Outcome 5 Hughston VAS}

patellofemoral score ( 28 to 100: best outcome).

Review: Surgical versus non-surgical interventions for treating patellar dislocation

Comparison: I Surgical versus non-surgical management

Outcome: 5 Hughston VAS patellofemoral score (28 to 100: best outcome)

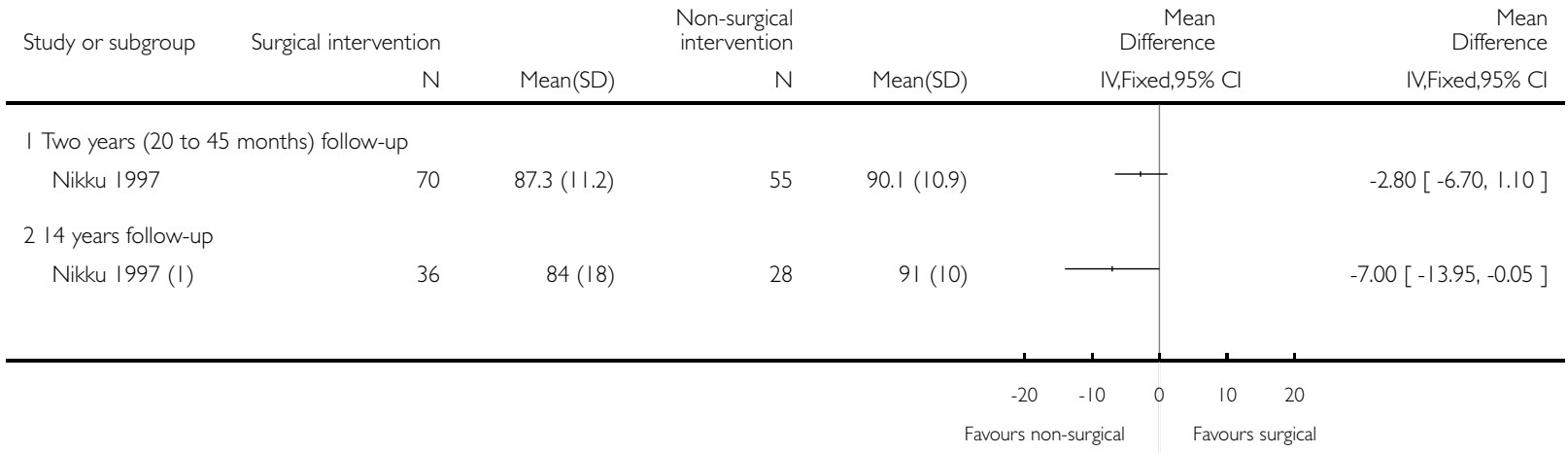

( ) Subgroup: children only 


\section{Analysis I.6. Comparison I Surgical versus non-surgical management, Outcome 6 Kujala patellofemoral disorders score (0 to 100: best outcome).}
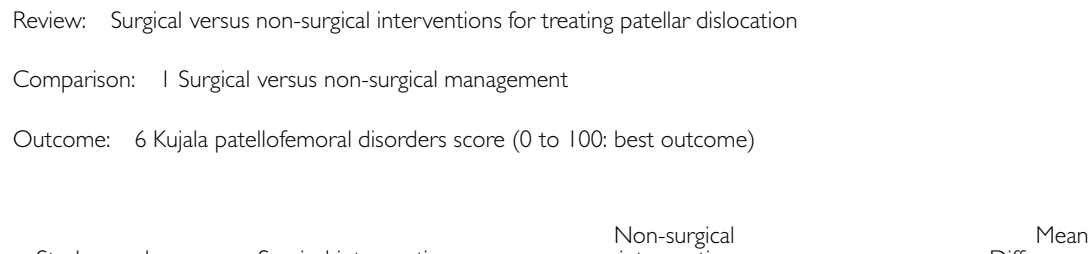

Difference

Weight

Mean

Difference

IV,Random,95\% Cl IV,Random,95\% Cl

I Two to five years follow-up

Bitar 2012 (I)

$21 \quad 88.9(10.4)$

$20 \quad 70.8(19.2)$

Camanho 2009 (2)

$1791.23(5.01)$

$16 \quad 69.06(14.02)$

Christiansen 2008

$42 \quad 84.6(17.5)$

35

78.1 (15.9)

Petri 2013

$12 \quad 87.5(13.3)$

$81.3(19.2)$

92

79

IV,Random, $95 \% \mathrm{Cl}$

Subtotal (95\% CI)

Heterogeneity: $\mathrm{Tau}^{2}=52.58 ; \mathrm{Chi}^{2}=10.36, \mathrm{df}=3(\mathrm{P}=0.02) ;\left.\right|^{2}=71 \%$

Test for overall effect: $Z=3.17(P=0.0015)$

2 Six to nine years follow-up

\begin{tabular}{|c|c|c|}
\hline Nikku 1997 & 7081.54 (18.09) & $5788.11(10.76)$ \\
\hline Sillanpaa 2009 (3) & $91(13)$ & $90(9.8)$ \\
\hline
\end{tabular}

Subtotal (95\% CI)

88

79

Heterogeneity: $\mathrm{Tau}^{2}=18.42 ; \mathrm{Chi}^{2}=2.80, \mathrm{df}=\mathrm{I}(\mathrm{P}=0.09) ; \mathrm{I}^{2}=64 \%$

Test for overall effect: $Z=0.87(P=0.39)$

314 years follow-up

Nikku 1997 (4)

36

$83(18)$

28

$84(13)$

36

28

Subtotal (95\% CI)

Heterogeneity: not applicable

Test for overall effect: $Z=0.26(P=0.80)$

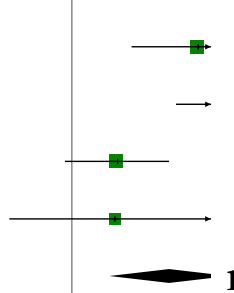

$25.3 \%$

$18.10[8.58,27.62]$

$29.0 \% \quad 22.17[14.90,29.44]$

$28.7 \%$

$6.50[-0.97,13.97]$

$17.0 \%$

$6.20[-9.09,21.49]$

$100.0 \% 13.93[5.33,22.53]$

$56.1 \%$

$-6.57[-11.65,-1.49]$

$43.9 \%$

$1.00[-6.27,8.27]$

$100.0 \%-3.25[-10.61,4.11]$

$100.0 \%$

$-1.00[-8.60,6.60]$

$100.0 \%-1.00[-8.60,6.60]$

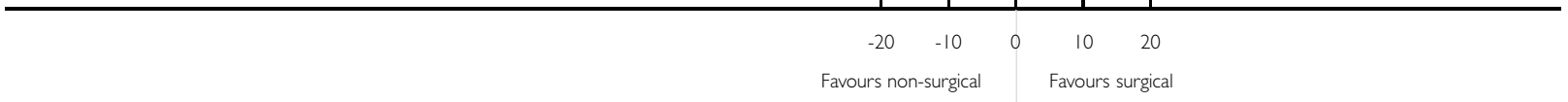

( I) 2 participants in non-surgical group had bilateral involvement - data for 20 knees of 18 participants

(2) Standard deviation values obtained from authors in 2010 (Bitar A, Personal communication)

(3) Standard deviation values obtained from authors in 2010 (Sillanpaa P, personal communication)

(4) Children only subgroup 
Analysis I.7. Comparison I Surgical versus non-surgical management, Outcome 7 Kujala patellofemoral disorders score (0 to 100: best outcome): subgroup analysis.

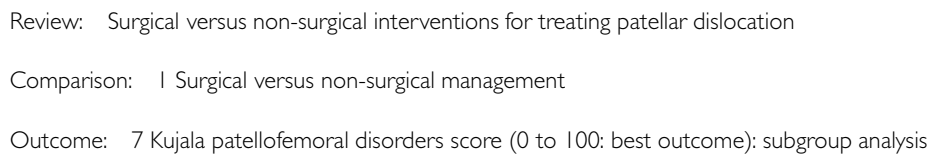

\begin{tabular}{|c|c|c|c|c|c|c|c|c|}
\hline \multirow[t]{2}{*}{ Study or subgroup } & \multirow{2}{*}{$\begin{array}{r}\text { Surgical } \\
N\end{array}$} & \multicolumn{3}{|c|}{ Non-Surgical } & \multicolumn{2}{|c|}{$\begin{array}{r}\text { Mean } \\
\text { Difference }\end{array}$} & \multirow[t]{2}{*}{ Weight } & \multirow{2}{*}{$\begin{array}{r}\text { Mean } \\
\text { Difference } \\
\text { IV,Fixed,95\% Cl }\end{array}$} \\
\hline & & Mean(SD) & $\mathrm{N}$ & Mean(SD) & & $\mathrm{ed}, 95 \% \mathrm{Cl}$ & & \\
\hline \multicolumn{9}{|l|}{ I Male } \\
\hline Bitar 2012 & 12 & $91.6(6.7)$ & 9 & $76.1(17.2)$ & & + & $61.5 \%$ & $15.50[3.64,27.36]$ \\
\hline Subtotal (95\% CI) & 12 & & 9 & & & $<$ & $61.5 \%$ & $15.50[3.64,27.36]$ \\
\hline \multicolumn{9}{|c|}{ Heterogeneity: not applicable } \\
\hline \multicolumn{9}{|c|}{ Test for overall effect: $Z=2.56(P=0.010)$} \\
\hline \multicolumn{9}{|l|}{2 Female } \\
\hline Bitar 2012 & 9 & $85.2(13.5)$ & 11 & $66.5(20.5)$ & & 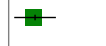 & $38.5 \%$ & $18.70[3.71,33.69]$ \\
\hline Subtotal (95\% CI) & 9 & & 11 & & & $<$ & $38.5 \%$ & $18.70[3.71,33.69]$ \\
\hline \multicolumn{9}{|c|}{ Heterogeneity: not applicable } \\
\hline \multicolumn{9}{|c|}{ Test for overall effect: $Z=2.45(P=0.014)$} \\
\hline Total (95\% CI) & 21 & & 20 & & & $<$ & $100.0 \%$ & $16.73[7.43,26.03]$ \\
\hline \multicolumn{9}{|c|}{ Heterogeneity: Chi $^{2}=0.11, d f=I(P=0.74) ;\left.\right|^{2}=0.0 \%$} \\
\hline \multicolumn{9}{|c|}{ Test for overall effect: $Z=3.53(P=0.00042)$} \\
\hline \multicolumn{9}{|c|}{ Test for subgroup differences: $\mathrm{Chi}^{2}=0.1 \mathrm{l}, \mathrm{df}=\mathrm{I}(\mathrm{P}=0.74), \mathrm{I}^{2}=0.0 \%$} \\
\hline & & & & -100 & -50 & 50 & 100 & \\
\hline & & & & Favours Non & Surgica & Favours & Surgical & \\
\hline
\end{tabular}


Analysis I.8. Comparison I Surgical versus non-surgical management, Outcome 8 Return to former activities: work and sports.

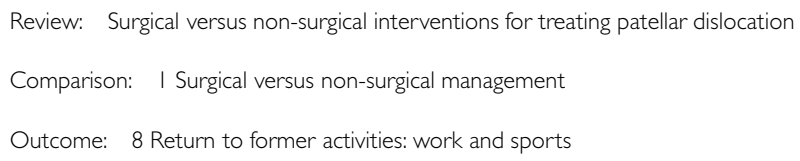

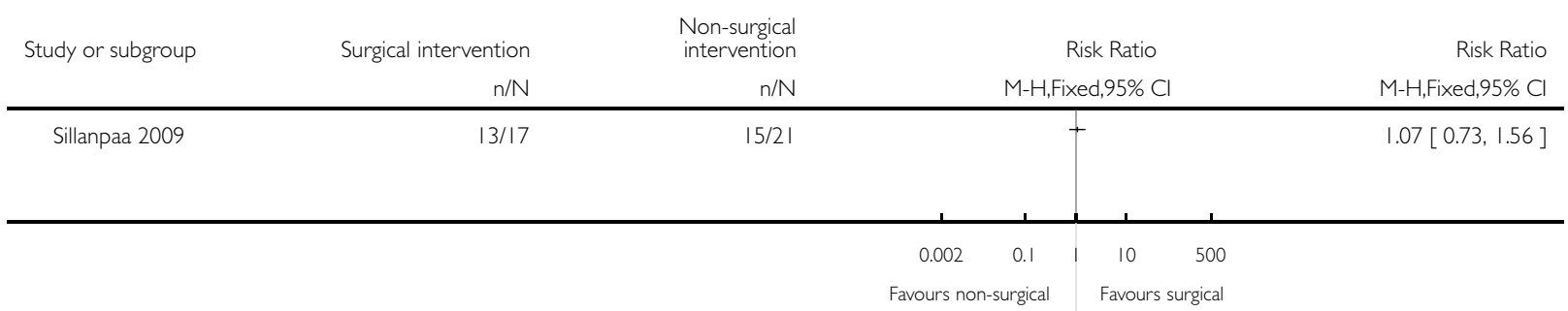

\section{Analysis I.9. Comparison I Surgical versus non-surgical management, Outcome 9 Knee pain (VAS 0 to I0:} worst outcome).

Review: Surgical versus non-surgical interventions for treating patellar dislocation

Comparison: I Surgical versus non-surgical management

Outcome: 9 Knee pain (VAS 0 to 10: worst outcome)

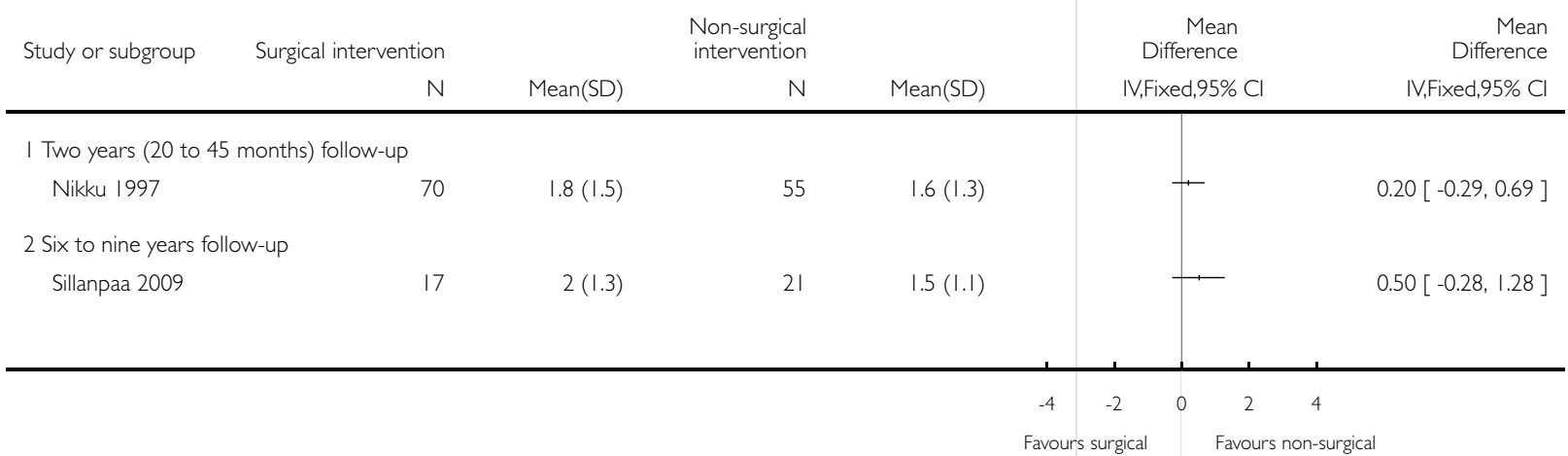


Analysis I.I0. Comparison I Surgical versus non-surgical management, Outcome I0 Patient satisfaction (reported good or excellent).

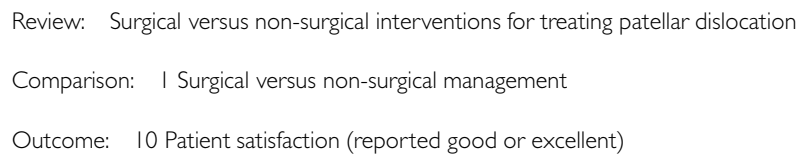

-

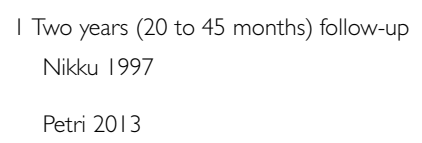

$\begin{array}{rr}49 / 70 & 39 / 55 \\ 8 / 11 & 4 / 8\end{array}$

+

\section{Subtotal (95\% CI)}

$81 \quad 63$

Total events: 57 (Surgical intervention), 43 (Non-surgical intervention)

Heterogeneity: $\mathrm{Chi}^{2}=0.89, \mathrm{df}=\mathrm{I}(\mathrm{P}=0.35) ; \mathrm{I}^{2}=0.0 \%$

Test for overall effect: $Z=0.28(P=0.78)$

2 Six to nine years follow-up

$$
\begin{array}{lll}
\text { Nikku } 1997 & 47 / 70 & 46 / 57
\end{array}
$$

$\begin{array}{lll}\text { Subtotal (95\% CI) } & \mathbf{7 0} & 57\end{array}$

Total events: 47 (Surgical intervention), 46 (Non-surgical intervention)

Heterogeneity: not applicable

Test for overall effect: $Z=1.74(P=0.082)$

314 years follow-up

$$
\text { Nikku } 1997 \text { (I) }
$$

Subtotal (95\% CI)

$21 / 32 \quad 21 / 28$

32

Total events: 2 I (Surgical intervention), 2 I (Non-surgical intervention)

Heterogeneity: not applicable

Test for overall effect: $Z=0.79(P=0.43)$

$4 / 8$

63

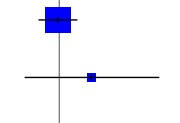

$100.0 \%$

$0.83[0.68,1.02]$

$100.0 \%$

$100.0 \%$

$0.88[0.63,1.22]$

28

7

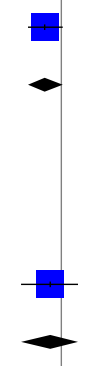

$100.0 \%$

$0.88[0.63,1.22]$

(I) Children only subgroup 


\section{Analysis I.I I. Comparison I Surgical versus non-surgical management, Outcome II Number of participants sustaining recurrent patellar subluxation.}

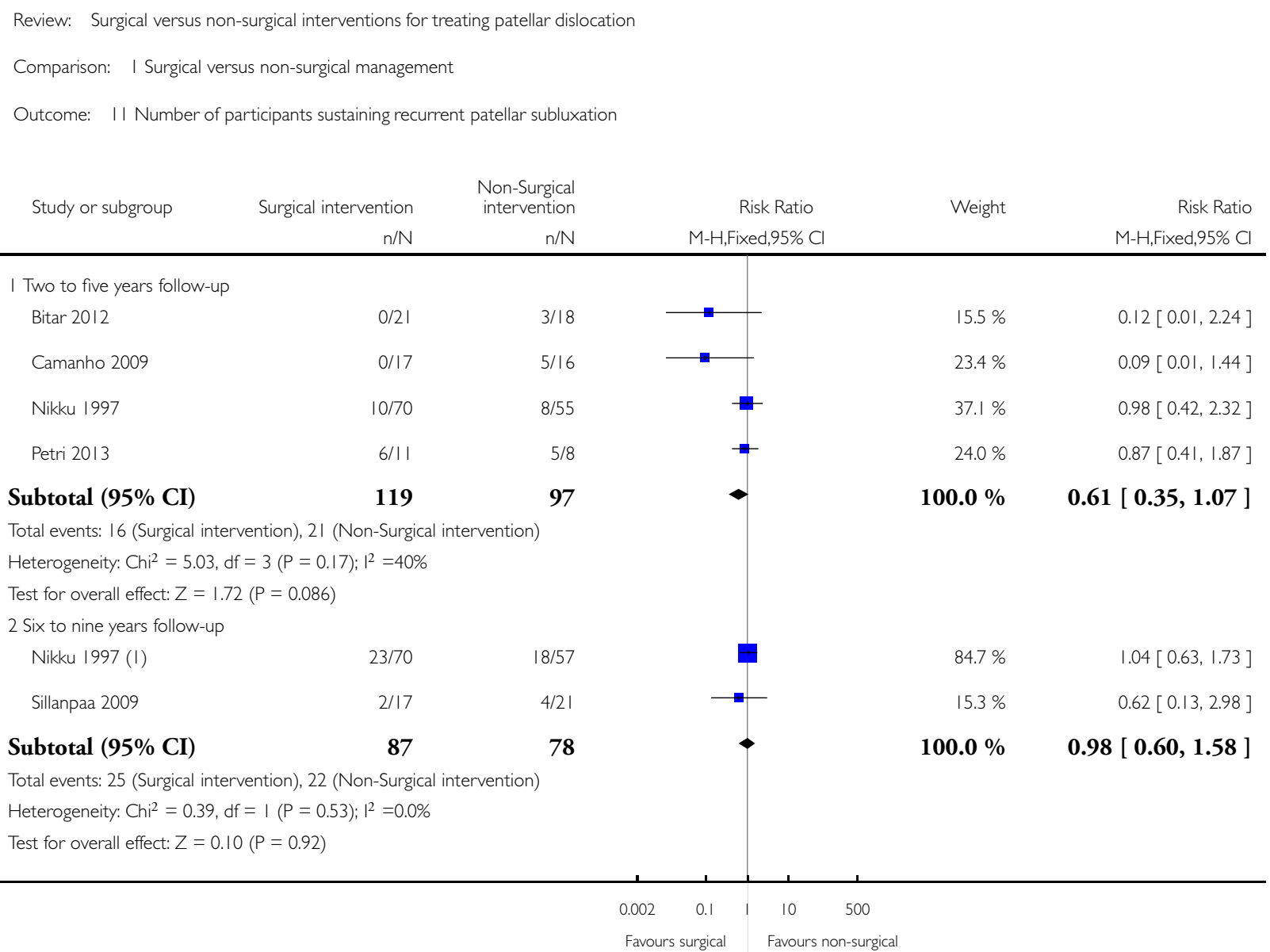

(I) Reported data were for dislocations, and all episodes of instability. Event data entered are all episodes minus dislocations 


\section{Analysis I.13. Comparison I Surgical versus non-surgical management, Outcome I 3 Number of participants who underwent subsequent surgery.}

\begin{tabular}{|c|c|c|c|c|c|}
\hline \multicolumn{6}{|c|}{ Comparison: I Surgical versus non-surgical management } \\
\hline \multicolumn{6}{|c|}{ Outcome: 13 Number of participants who underwent subsequent surgery } \\
\hline \multirow[t]{2}{*}{ Study or subgroup } & Surgical intervention & $\begin{array}{l}\text { Non-Surgical } \\
\text { intervention }\end{array}$ & Risk Ratio & \multirow[t]{2}{*}{ Weight } & Risk Ratio \\
\hline & $n / N$ & $n / \mathrm{N}$ & M-H,Fixed,95\% Cl & & M-H,Fixed,95\% Cl \\
\hline \multicolumn{6}{|c|}{ I Two years (20 to 45 months) follow-up } \\
\hline Nikku 1997 & $12 / 70$ & $9 / 55$ & & $100.0 \%$ & $1.05[0.48,2.31]$ \\
\hline Subtotal $(95 \% \mathrm{CI})$ & 70 & 55 & & $100.0 \%$ & $1.05[0.48,2.31]$ \\
\hline \multicolumn{6}{|c|}{ Total events: 12 (Surgical intervention), 9 (Non-Surgical intervention) } \\
\hline \multicolumn{6}{|c|}{ Heterogeneity: not applicable } \\
\hline \multicolumn{6}{|c|}{ Test for overall effect: $Z=0.12(P=0.91)$} \\
\hline \multicolumn{6}{|l|}{2 Six to nine years follow-up } \\
\hline Nikku 1997 & 20/70 & $13 / 57$ & & $82.0 \%$ & $1.25[0.68,2.29]$ \\
\hline Sillanpaa 2009 & $0 / 17$ & $3 / 21$ & - & $18.0 \%$ & $0.17[0.01,3.16]$ \\
\hline Subtotal $(95 \% \mathrm{CI})$ & 87 & 78 & > & $100.0 \%$ & $1.06[0.59,1.89]$ \\
\hline \multicolumn{6}{|c|}{ Total events: 20 (Surgical intervention), 16 (Non-Surgical intervention) } \\
\hline \multicolumn{6}{|c|}{ Heterogeneity: $\mathrm{Chi}^{2}=1.79, \mathrm{df}=1(P=0.18) ;\left.\right|^{2}=44 \%$} \\
\hline \multicolumn{6}{|c|}{ Test for overall effect: $Z=0.19(P=0.85)$} \\
\hline \multicolumn{6}{|l|}{314 years follow-up } \\
\hline Nikku 1997 (I) & $16 / 36$ & $11 / 28$ & & $100.0 \%$ & $1.13[0.63,2.04]$ \\
\hline Subtotal $(95 \% \mathrm{CI})$ & 36 & 28 & > & $100.0 \%$ & $1.13[0.63,2.04]$ \\
\hline \multicolumn{6}{|c|}{ Total events: 16 (Surgical intervention), II (Non-Surgical intervention) } \\
\hline \multicolumn{6}{|c|}{ Heterogeneity: not applicable } \\
\hline Test for overall effect: $Z=$ & $(P=0.68)$ & & & & \\
\hline
\end{tabular}

(I) Children only subgroup 


\section{A P P E N D I C E S}

\section{Appendix I. Search strategies (August 2010 to October 2014)}

\section{CENTRAL (Wiley Online Library)}

\#1 MeSH descriptor: [Patellar Dislocation] this term only (28)

\#2 MeSH descriptor: [Patella] this term only (244)

\#3 MeSH descriptor: [Dislocations] this term only (224)

\#4 (\#2 and \#3) (4)

\#5 patell* near/3 (dislocat* or sublux* or instability):ti,ab,kw (80)

\#6 (\#1 or \#4 or \#5) in Trials (67)

\section{MEDLINE (Ovid interface)}

1 Patellar Dislocation/ (562)

2 Patella/ and (Dislocations/ or Joint Instability/) (1410)

3 (patell\$ adj3 (dislocat\$ or sublux\$ or instability)).tw. (2068)

4 or/1-3 (2784)

5 trochleoplasty.tw. (61)

6 Roux-Goldthwaite.tw. (2)

7 (tibial tubercle adj3 transfer).tw. (64)

8 quadricepsplasty.tw. (101)

9 (medial patellofemoral ligament adj3 (reconstruction or repair)).tw. (216)

10 medial reefing.tw. (16)

11 medial augmentation.tw. (2)

12 lateral release.tw. (468)

13 Orthopedics/ (16598)

14 exp Surgical Procedures, Operative/ (2466406)

15 su.fs. (1624851)

16 surg\$.tw. (1394491)

17 operat\$.tw. (784688)

18 realign\$.tw. (3345)

19 exp Rehabilitation/ (156321)

20 exp Physical Therapy Modalities/ (129593)

21 "Physical Therapy (Specialty)"/ (2210)

22 Braces/ (4560)

23 Immobilization/ (11852)

24 rh.fs. (172029)

25 rehabilitat\$.tw. (114582)

26 physiotherapy.tw. (12685)

27 physical therapy.tw. (11376)

28 (non-surg\$ or nonsurg\$ or non-operat\$ or nonoperat\$ or conserv\$).tw. (358060)

29 (immobili $\$$ or therap\$ or exercis\$ or taping or tape $\$$ or bracing or brace\$ or manual therapy or electrotherap\$).tw. (2231573)

30 or/5-29 (6066183)

31 and/4,30 (2135)

32 Randomized controlled trial.pt. (396976)

33 Controlled clinical trial.pt. (90468)

34 randomized.ab. (316328)

35 placebo.ab. (162763)

36 Drug therapy.fs. (1773912)

37 randomly.ab. (226880)

Surgical versus non-surgical interventions for treating patellar dislocation (Review)

Copyright $\Subset 2015$ The Cochrane Collaboration. Published by John Wiley \& Sons, Ltd. 
38 trial.ab. (330054)

39 groups.ab. (1427182)

$40 \mathrm{or} / 32-39(3505162)$

41 exp Animals/ not Humans/ (4075570)

4240 not 41 (3010421)

43 and $/ 31,42(238)$

$44\left(201008^{*}\right.$ or $20109^{*}$ or $201010^{*}$ or $201011^{*}$ or $201012^{*}$ or $2011^{*}$ or $2012^{*}$ or $2013^{*}$ or $\left.2014^{*}\right)$.ed. (4160900)

4543 and $44(66)$

\title{
EMBASE (Ovid interface)
}

1 Patellar Dislocation/ (1667)

2 Patella/ and Dislocation/ (427)

3 (patell\$ adj3 (dislocat\$ or sublux\$ or instability)).tw. (2201)

4 or/1-3 (2985)

5 Clinical trial/ (834564)

6 Randomized controlled trial/ (351271)

7 Randomization/ (63524)

8 Single blind procedure/ (18900)

9 Double blind procedure/ (115714)

10 Crossover procedure/ (40361)

11 Placebo/ (246816)

12 randomi?ed controlled trial\$.tw. (104177)

13 rct.tw. (14914)

14 random allocation.tw. (1341)

15 randomly allocated.tw. (20881)

16 allocated randomly.tw. (1949)

17 (allocated adj2 random).tw. (717)

18 single blind\$.tw. (14712)

19 double blind\$.tw. (143861)

20 ((treble or triple) adj blind\$).tw. (395)

21 placebo\$.tw. (202854)

22 Prospective study/ (263230)

23 or/5-22 (1388817)

24 Case study/ (28194)

25 case report.tw. (265152)

26 Abstract report/ or Letter/ (903050)

27 or/24-26 (1190552)

2823 not 27 (1350752)

29 limit 28 to human (1239498)

30 and/4,29 (174)

31 (201008* $^{*}$ or $201009^{*}$ or $201010^{*}$ or $201011^{*}$ or $201012^{*}$ or $2011^{*}$ or $2012^{*}$ or $2013^{*}$ or $\left.2014^{*}\right)$.dd. (5911880)

3230 and $31(61)$

\section{CINAHL (NHS NICE Healthcare Databases)}

\author{
1 PATELLA DISLOCATION/ (127) \\ 2 PATELLA/ (898) \\ 3 DISLOCATIONS/ (1789) \\ 4 AND/ 2,3 (47) \\ 5 (patell* ADJ3 dislocat*).ti,ab (180) \\ 6 (patell* ADJ3 sublux*).ti,ab (79) \\ 7 (patell* ADJ3 instability).ti,ab (153)
}

Surgical versus non-surgical interventions for treating patellar dislocation (Review)

Copyright $\odot 2015$ The Cochrane Collaboration. Published by John Wiley \& Sons, Ltd. 
8 OR/5-7 (328)

9 CLINICAL TRIALS/ (80747)

10 EVALUATION RESEARCH/ (18181)

11 COMPARATIVE STUDIES/ (70989)

12 CROSSOVER DESIGN/ (8841)

13 OR/9-12 (170155)

14 (clinical OR controlled OR comparative OR placebo OR prospective OR randomised OR randomized).ti,ab (397157)

15 (trial OR study).ti,ab (581026)

16 AND/14,15 (207828)

17 random*.ti,ab (114047)

18 (allocat* OR allot* OR assign* OR basis* OR divid* OR order*).ti,ab (137570)

19 AND/17,18 (32446)

20 (singl* OR doubl* OR trebl* OR tripl*).ti,ab (84143)

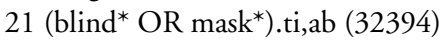

22 AND/20,21 (17579)

23 ("cross over" OR cross-over OR crossover).ti,ab (7010)

24 (allocat* OR allot* OR assign* OR divid*).ti.ab (57)

25 (condition* OR experiment* OR intervention* OR treatment* OR therap* OR control* OR group*).ti,ab (798852)

26 AND/23-25 (1)

27 OR/13,16,19,22,26 (338160)

$28 \mathrm{OR} / 4,8(346)$

$29 \mathrm{AND} / 1,28(73)$

\section{AMED (NHS NICE Healthcare Databases)}

1 Patella/ (364)

2 Dislocations/ (459)

3 AND/1-2 (17)

4 (dislocat* OR sublux* OR instability).ti,ab (2937)

5 patell*.ti,ab (1295)

$6 \mathrm{AND} / 4,5$ (93)

$7 \mathrm{OR} / 3,6(96)$

\section{Other databases}

We searched the following databases for 'patella' and 'dislocation' terms:

- Health Management Information Consortium (NHS NICE Healthcare Databases) (0)

- Physiotherapy Evidence Database (PEDro) (4)

- Zetoc (MetLib University of East Anglia) (344)

- OpenGrey (0)

- WHO International Clinical Trials Registry Platform (7)

- Current Controlled Trials (9)

- UKCRN Portfolio Database (0)

- National Technical Information Service (1)

- National Research Register Archive (164) 


\section{Appendix 2. Previous search results}

A total of 1328 references were produced by the search strategy (see Appendix 1). Two review authors assessed them against the eligibility criteria, identifying a total of 12 studies that appeared pertinent to the research question. Full texts of these studies were ordered and five trials were confirmed as satisfying the inclusion criteria and were subsequently included in the review.

\section{F E E D B A C K}

\section{Presentational errors, I 7 November 201 I}

\section{Summary}

We have used this new review for teaching purposes in our post-graduate programme and realized that Figure 3 is wrong and does not match with Analysis 1.1:

1. It does not contain all the graphical elements for sections 1.1.1 and 1.1.2.

2. The point estimates and diamonds shown are on the wrong side (i.e. favouring non-surgical interventions).

3. In Analysis 1.3, the label of the $\mathrm{x}$-axis (exp/control) differs from the other forest plots.

We hope these errors can be corrected.

\section{Reply}

We thank Dr von Elm for contacting us and are glad with his use of our review. His observations are all correct. Regarding the mismatch between Analysis 1.1 and Figure 1, errors of reproduction appear to have occurred at some point in the processing of the review, including in the generation of the pdf files for publication. We have revised the scale of Analysis 1.1 and checked that Figure 1 accurately reflects this in RevMan before resubmission for publication. The Managing Editor of the Bone, Joint and Muscle Trauma Group has notified the RevMan support team and Wiley of this problem.

The inconsistent labelling of Analysis 1.3 has now been changed to read "surgical" : "non-surgical" for consistency.

\section{Contributors}

Comment from: Dr Erik von Elm

Reply from: Professor William Gillespie and Dr Helen Handoll (Cochrane, Bone, Joint and Muscle Trauma Group), 22 November 2011

\section{WHAT'S NEW}

Last assessed as up-to-date: 13 October 2014.

\begin{tabular}{l|l|l}
\hline Date & Event & Description \\
\hline 24 December 2014 & $\begin{array}{l}\text { New citation required but conclusions have not } \\
\text { changed }\end{array}$ & $\begin{array}{l}\text { Two new studies (Bitar 2012; Petri 2013) included. } \\
\text { One study (Palmu 2008) included in the previous ver- } \\
\text { sion was found to be a subgroup (children only) of } \\
\text { another included study (Nikku 1997). } \\
\text { 'Summary of findings' table incorporated. }\end{array}$ \\
\hline
\end{tabular}

Surgical versus non-surgical interventions for treating patellar dislocation (Review) 


\section{H I S T O R Y}

Protocol first published: Issue 4, 2009

Review first published: Issue 11, 2011

\begin{tabular}{lll}
\hline Date & Event & Description \\
\hline 22 November 2011 & Feedback has been incorporated & Feedback incorporated and minor changes made. \\
\hline
\end{tabular}

\section{CONTRIBUTIONS OFAUTHORS}

Caroline Hing and Toby Smith co-ordinated and conceived the protocol, and, with assistance of Lesley Gillespie from the Cochrane Bone, Joint and Muscle Trauma Group, designed the search strategy. Fujian Song provided guidance on methodological and statistical analysis during the development of the protocol. Caroline Hing, Toby Smith and Simon Donell provided a clinical perspective during the protocol development and review preparation. Caroline Hing, Toby Smith, Fujian Song and Simon Donell designed and wrote the protocol.

Joanne Elliott, Toby Smith and Caroline Hing performed the search strategy. Toby Smith and Caroline Hing screened the search results and identified the studies, extracted the data and prepared the data extraction table for analysis. Toby Smith and Fujian Song analysed the data. Caroline Hing, Toby Smith and Simon Donell provided a clinical perspective during the full review development and preparation. Caroline Hing, Toby Smith, Fujian Song and Simon Donell all revised and agreed the full review.

Caroline Hing is the guarantor of the protocol and full review.

\section{DECLARATIONS OF INTEREST}

Toby O Smith: none known.

Simon Donell: was an investigator of a trial included in the review (Nikku 1997). This trial was assessed independently by other review authors.

Fujian Song: none known.

Caroline B Hing: none known. 


\section{SOURCES OF SUPPORT}

\section{Internal sources}

- St George's University, London, UK, UK.

- University of East Anglia, Norwich, UK.

- Norfolk and Norwich University Foundation Hospital NHS Trust, Norwich, UK.

\section{External sources}

- No sources of support supplied

\section{DIFFERENCES BETWEEN PROTOCOLANDREVIEW}

1. In the review, two additional outcome measures were reported as secondary outcome measures. They were patient-reported satisfaction and the subsequent requirement for surgery. Both outcomes were reported by a number of original research studies, and therefore considered important to include in the final review.

2. We revised our 'Risk of bias' assessment to comply with the new guidance in Higgins 2011.

3. We presented the data for primary and secondary outcomes in subgroups characterised by length of follow-up with data presented from each follow-up interval presented rather than the final follow-up datapoint in each included study.

4. We incorporated a 'Summary of findings' table to comply with new guidance in Higgins 2011.

\section{NDEX TERMS}

\section{Medical Subject Headings (MeSH)}

Patellar Dislocation [surgery; *therapy]; Randomized Controlled Trials as Topic

\section{MeSH check words}

Adolescent; Adult; Child; Humans; Young Adult 